\title{
DYNAMIC RESPONSE OF A CYLINDRICAL SHELL IMMERSED IN A POTENTIAL FLUID
}

Garth E. Cummings

(Ph.D. Thesis)

April 28, 1978

Work performed under the auspices of the U.S. Department of Energy by the UCLLL under contract number W-7405-ENG-48.

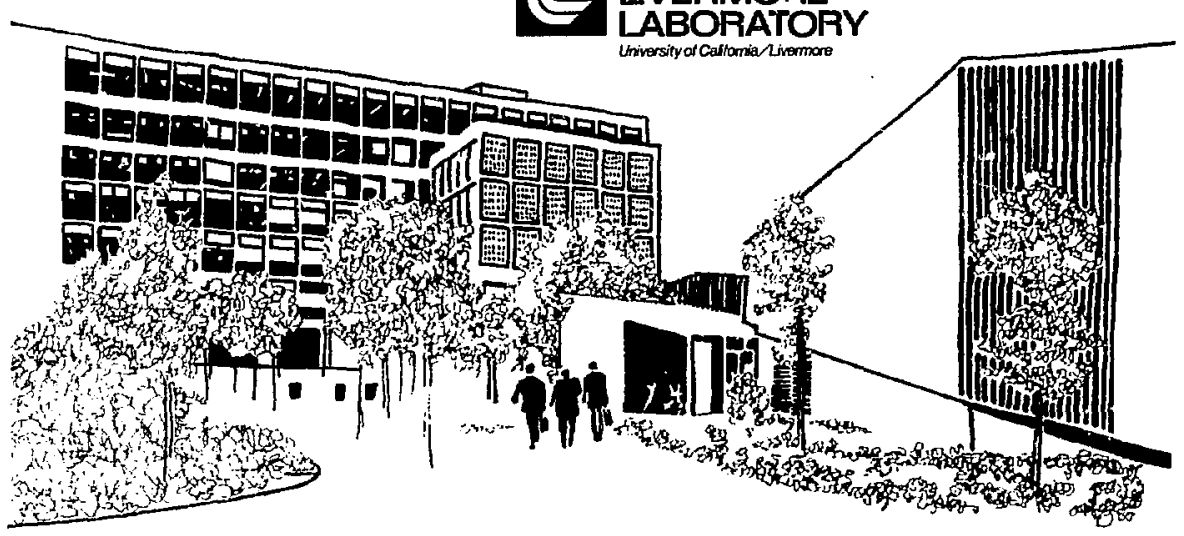

\section{IL LAWRENCE LIVERMORE LABORATORY}

\section{MASTER}




\section{NOTICE}

"Thl report we prepared a an account of work sponzored by the United States Govemment. Neikher the United States nor the United Statea Depertment of Energy, nor any of thelr comployees, nor any of their contractors, subcontractors, or their employees, mokes eny wartanty. expres or Implted, or asumes any legl lubility or ces ondbility for the accurscy, completenes or usefulnes of any information, apparatur, product or proces dlaclosed, or represents that its une would not infringe privately-owned rights."

\section{NOTICE}

Reference to a company or product name does not imply approval or recommendation of the product by the University of California or the L.S. Department of Energy to the exclusion of others that may be suitable.

Printed in the United States of America Avallable from

National Technical Information Service U.S. Department of Commerce 5285 Port Royal Rond Springlield, VA 22161 Price: Printed Copy \$ : Mitrofiche $\$ 3.00$

Pepe Range

Domestic

$001-025$

026-050

$051-075$

$$
\text { Price }
$$

$076-100$

101-125

$126-150$

$151-175$

176-200

201-225

226-250

$251-275$

276-300

301-325

54.00
4.50
5.25
6.00
6.50
7.25
8.00
9.00
9.25
9.50
10.75
11.00
11.75

$$
\begin{aligned}
& \text { Pege Fanpe } \\
& \hline 326-350 \\
& 351-375 \\
& 376-400 \\
& 401-425 \\
& 426-450 \\
& 451-475 \\
& 476-500 \\
& 501-525 \\
& 526-550 \\
& 551-575 \\
& 576-600 \\
& 601-4 p
\end{aligned}
$$

Domestic

Pries

$\$ 12.00$

12.50

13.00

I3.25

14.00

14.50

15.00

15.25

15.50

16.25

16.50

1 Ade 32.50 for exch eddiltond 100 cure inarnment from 601 pagel up. 


\section{屿 \\ LAWRENCE LIVERMOGE IABORATORY \\ University of Calfomia Livermore, California 94550}

UCRL-52464

\section{DYNAMIC RESPONSE OF A CYLINDRICAL SHELL IMMERSED IN A POTENTIAL FLUID}

Garth E. Cummings

(Ph.D. Thesis)

April 28, 1978

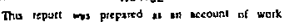

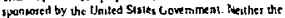


Abstract-1

Introduction-...... 2

Analysis--

Shel1 Equations-- 7

Fluid Equations-1-..- 9

Calculational Method-_. 11

Shel1 Equations:- 12

Fluid Equations--_-1 13

Calculational Technique-- 14

Experimenta] Verification-16

App]ication of Numerical Technique-- 24

Conciusion--

Nomenclature--10 30

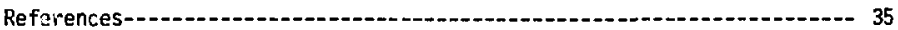

Appendix A - Derivation of the Governing Equations-- 37

She11 Equations-1 37

Fluid Equations-- 54

Analytical Solution-1 58

References for Appendix A- 63

Appendix B - Development of Equations used for Computation---:--:-- 64

Basic Strategy-an 64

Shell Equations-and 65

Fluid Equations-1 77

References for Appendix B-1..- 83 


\section{CONTENTS (cont'd)}

Appendix C - Description of Experiment-1

Description of Apparatus- 84

Instrumentation and Data Reduction- 88

Measuremerit Techniques-.. 92

Experimental Results-_. 96

References for Appendix C-_...... 109

Appendix D - The Computer Code SHELVIB-

Listing of Computer Code SHELVIB-_.

Sample Input-_

Sample Output-139

References for Appendix D. 
DYNAMIC RESPONSE OF A CYLINDRICAL SHELL

IMMERSED IN A POTENTIAL FLUID

\section{ABSTRACT}

A numerical solution technique is presented for determining the dynamic response of a thin, elastic, circular, cylindrical shell of constant wall thickness and density, immersed in a potential fluid. The shell may be excited by an arbitrary radial forcing function with a specified time history and spatial distribution. In addition, a pressure history may be specified over a segment of the fluid outer boundary. Any of the natural shell end conditions may be prescribed.

A numerical instability prevented direct solutions where the ratio of the hydrodynamic forces to shell inertial forces is greater than two. This instability is believed to be the result of the weak coupling between the equations describing the fluid to those describing the shell. To circumvent this instability, an effective mass was ca?culated and added to the she11.

Comparison of numerical to experimental results are made using a $1 / 12$ scale model of a nuclear reactor core support barrel. Natural frequencies and modes are determined for this model in air, water, and oil. The computed frequencies compare to experimental results to within $15 \%$.

We illustrate the use of this numerical technique by comparing it to an analytical solution for shell beam modes and resolve an uncertainty in the anajytical technique concerning the proper effective mass to use. 


\section{INTROOUCTION}

Whenever solids move in contact with fluids, the resisting pressure of the fluid should be taken inis account. Traditionally, this pressure is assumed proportional to the acce?eration of the moving solid relative to the fluid and is accounted for by assigning an increased density to the solid. Thus, an increased or "effective mass" is incorporated into the equation of motion of the solid structure to account sor this fluid influence. For shells this effective mass is a function of mode number, shel1 radius, thickness, length, and proximity of neighboring structures. As will be shown, the effect of the fruis on the motion of a cubmerged shell can be dramatic with reductions by a factc: " of thrce of the natural frequencies.

Early workers in this field [i] used this effective mass technique with modal analysis to analyze sheils subl,erged in a fluid. Closed form solutions vere obtained for simply-supported cylinders in infinite fluids. More recently, sinijlar techniques hase been appiad to coaxial cylindrical shells [2-6]. The approach used is to couple the fluid to the she11 by forcing the radial velocity of the sheit to equal that of the fluid at the she11 wall and the dynamic pressure in the fluir. to eqtil the loading on the she17. The acoustic wave equation is used to represent the fluid (assuming stationary fluid and small motions) in corjuncticn with the equations of motion of thin shells.

In addition to the fluid's effect on the vibration frequencies of a submerged shell, the fluid can also act as a vehicle for the transmission of forces to cause vitrations. Such is the case of the core support barrel of a nuclear reactor where the fluid flowing down the annulus between the core support barrel and pressure vessel wall imparts bath a random force due to turbulence and a deterministic force sue to pump pulsations. The 
dynamic response of the fluid-filled tanks $i_{1}$ rockets used in the space program is another area where fluid-shell interaction is important.

Recent methods used todetermine a shell's response to these types of fluid frrces will now be outlined.

Bowers and Horvay [4] calculated the response of a simply-suoported shell to pulsating pressure in an encompassing water-filled annulus. The purmp induced pressure pulsations are imposed over a portion of the outer feriphery of the water annulus resulting in a time-dependent mixed boundary value problem since the remainder of the outer periphery is modeled as an inslexible wall. An approximate solution method which gives the pressure distribution in the water annulus is made pcssible by replacing the pressure pulsations with equivalent body forces in the annulus and thus converting the mixed boundary value pivisem into a forced vibration problem with homcigeneous boundary conditions. The solution of this forced vibration problem gives the pressure in the annulus and on the she11 wall. The shell equations are next solved by the normal mode method to obtain natural frequencies and displacements of the shell. This analysis is only applicable to simply-supported shells and does rat account for water inside the shell.

Au-Yang $[5,7]$ used a statistical method to predict the r.m.s. response of a shell surrounded by a water annulus and subjected to random excitation unifomly distributed about the shell. This method is based on finite element techniques and requires prior knowledge of the shell mode shapes and the cross-spectral density of the forcing function.

Berger and Palmer [8] have devised a numerical solution technique to determine the transient motion of an infinitely long, viscoelastic, cylindrical shell of arbitrary cross-section, surrounded by an infinite 
acoustic medium. They used finite difference tachniques to model the conformally mapped she 11 and acoustic equations. Laplace transformation was used to handle the time derivative.

Other, more comprehensive numerical techniques are described by Belytschko [9]. In genera1, these were developed to describe structural motion resulting from high energy impulse loading as might result from 2 nuclear reactor accident. They, thus, are tailored to find short term and nonlinear responses and require such small time steps as to make calculatinn to steacy-state impractical.

In our attempts to treat the fluid-shell problem analytically, a number of assumptions are made. The shell is assumed either infinitely long or simply-supported where in an actual installation neither is the case. Also, shell mode shapes in a fluid are assumed similar to those in vacuo. The latter has little effect on calculation of the natural frequencies, but presents difficulties when support clearances affect motion or where shell loads are being rositioned at she11 noies to minimize resprnses. The published numerical methous treat the shell and fluid as a discretized continuum and therefore circumvent these considerations but are tailored for short tem transient calculations, making computation to steady state prohibitively expensive.

We have developed a unique numerical solution technique which can accurately calculate a cylindrical shell's natural frequencies and mode shapes in potential fluids. This technique can be applied to thin, elastic, circular shells with constant wall thickness and density. The shell can be either in vacuo, filled with fluid, surrounded by fluid, or immersed in a fluid in a stiff, coaxial container. The length to radius ratio of the sheli? should be greater than one to predict natural 
frequencies to within $5 \%$ of those calculated by three dimensional theory. An arbitrary forcing function can be applied directly to the shell surface. In addition, a pressure history can be specified over a seoment of tive fluid's outer boundary, up to $1 / 4$ the shell's length. The fluid is modeled as a potential fluid and therefore, for an accurate calculation, the time period of the load or pressure change should be greater than the acoustic wave travel time over the fluid domain. Also the wave velocity of the $s^{2}$ ill should be less than the acoustic velocity of the liquid. Any of the natural boundary conditions can be prescribed on the ends of the she 11 and they may change with time.

This numericai solution technique is unique in several respects. Finite difference techniques were used to model the fiuid and she 11 with the potential fluid characterized by an elliptic Helmholtz type equation. The mixed boundary value prob?em was handled by a numerical technique that has been used in fiuid mechanics, but has not been eliployed in fluid-shell problems. A transient response approach was used to determine the short tam response as well as the natural frequencies and modes for the fluid-shell problem. Other transient resoonse codes are tailored to cumputed only the short time response to sudden phenomena such as occur with reactor accidents.

This numerica? solution technique can be used for design sensitivity S: $x_{i} i e s$, to check analytical solutions, or for numericai experimentation.

\section{ANAL YSIS}

This section describes the equations used to model the shell and the fluid regions inside and/or outside the shell. The geometry is described in Fig. 1. 


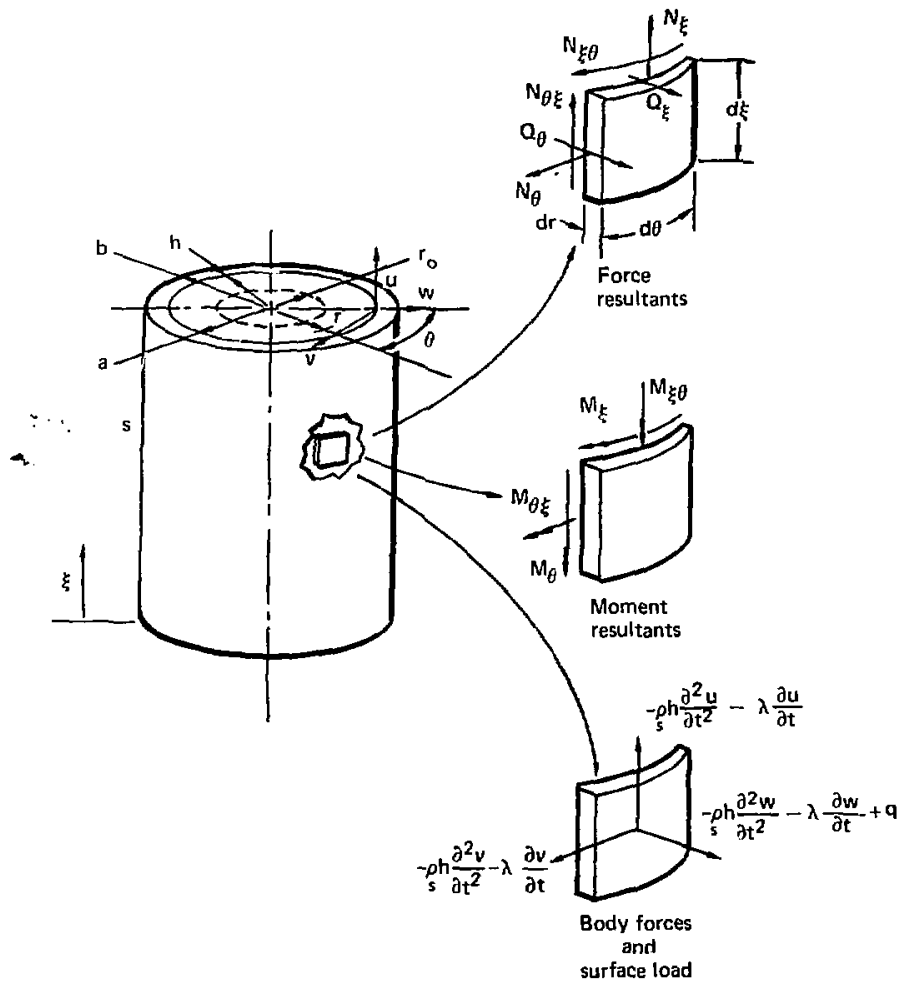

Fig. 1 Shell element, force and moment resultant diagrams. 


\section{Shell Equations}

The shell equations, as first postulated by Love, are a reduced form of the three dimensional theory of elasticity equations. In terms of finite difference techniques, noding of the shell in the radial direction is not required and this decreases computation time as compared to a three dimensional mesh. The basic assumptions used in formulation of the she 1 l equations are:

1. The shell is thin $(h / a<1 / 10)^{*}$

2. The deflections of the shell are small (1 inear theory holds)

3. The transverse normal stress is negligible.

4. The normals to the undefonmed midsurface of the shell remain strajght and normal to the deformed midsurface and they suffer no extension.

Sanders' solution method $[10,11,12]$ will be used. He emoloys the principle of virtual work to obtain the equilibrium equations for che shell element in Fig. 1. Leissa [13] demonstrates the close agreement between Sander's solution and that using the three dimensional theory of elasticity. By adding external loads and a D'Alembert term to these equilibrium equations we obtain the following equations of motion (see Appendix A).

$$
\begin{gathered}
\frac{\partial N_{\xi}}{\partial \xi}+\frac{\partial \bar{N}_{\xi \theta}}{\partial \theta}-\frac{1}{2 a} \frac{\partial \bar{M}_{\xi \theta}}{\partial \theta}-\frac{E h}{a} \frac{\partial^{2} u}{\partial \tau^{2}}-\lambda \sqrt{\frac{E}{\rho_{s}}} \frac{\partial u}{\partial \tau}=0 \\
\frac{\partial N_{\theta}}{\partial \theta}+\frac{\partial \bar{N}_{\xi \theta}}{\partial \xi}+\frac{3}{2 a} \frac{\partial \bar{M}_{\xi \theta}}{\partial \xi}+\frac{1}{a} \frac{\partial M_{\theta}}{\partial \theta}-\frac{E h}{a} \frac{\partial^{2} v}{\partial \tau^{2}}-\lambda \sqrt{\frac{E}{\rho_{s}} \frac{\partial v}{\partial \tau}}=0 \quad(1) \\
-N_{\theta}+\frac{1}{a}\left[\frac{\partial^{2} H_{\xi}}{\partial \xi^{2}}+2 \frac{\partial^{2} \bar{M}_{\xi \theta}}{\partial \xi \partial \theta}+\frac{\partial^{2} H_{\theta}}{\partial \theta^{2}}\right]-\frac{E h}{a} \frac{\partial^{2} w}{\partial \tau^{2}}-\lambda \sqrt{\frac{E}{\rho_{s}}} \frac{\partial w}{\partial \tau}+a q=0
\end{gathered}
$$

Nomenclature ifisted at end. 
We have introduced the dimensionless time variable $\tau \equiv\left[E / \rho_{s}\right]^{1 / 2} t / a$, a dimensionless axial space variable, $\xi \equiv x / a$, a viscous damping coefficient $\lambda$, and radial load $q$. The viscous damping coefficient is to include both structural and fluid damping which are small in most cases (see Table C-5 in Appendix C).

We simplify the analysis $[1,1,12]$ by describing (1) as a set of four dimensionless, coupled,partial differential equations in terms of $u, v, w, M_{\xi}, F_{F}$, and $\tau$. We do this by expanding the force and moment resultants, displacements, loads, and strains into Fourier series in the circumferential variable $\theta$. We then use a reference stress level, $\sigma_{0}$, to make the coefficients of the Fourier series dimensionless. For each rourier term, $n$, we obtain the following four coupled equations in which primes denote derivatives with respect to $\xi$ and dots with respect to I.

$$
\begin{aligned}
& \alpha_{1} u_{n}^{\prime \prime}+\alpha_{2} u_{n}+\alpha_{3} v_{n}^{\prime}+\alpha_{4} w_{n}^{\prime}=i_{n}+c \bar{u}_{n} \\
& \alpha_{5} u_{n}^{\prime}+\alpha_{6} v_{n}^{\prime \prime}+\alpha_{7} v_{n}+\alpha_{8} w_{n}^{\prime \prime}+\alpha_{9} w_{n}+\alpha_{10} M_{E n}=v_{n}+c v_{n} \\
& \alpha_{11} u_{n}{ }^{\prime}+\alpha_{12} v_{n}^{\prime \prime}+\alpha_{13} v_{n}+\alpha_{14} w_{n}^{\prime \prime}+\alpha_{15} w_{n}+\alpha_{16} M_{\xi n}^{\prime \prime}+\alpha_{17} M_{\xi n} \\
& =\ddot{w}_{n}+\dot{C w}_{n}-q_{n} \\
& \alpha_{18} v_{n}+\alpha_{19} w_{n} "+\alpha_{20} w_{n}+\alpha_{21} m_{\xi n}=0
\end{aligned}
$$

where

$$
u=\frac{a \sigma_{0}}{E} \sum_{n=0}^{\infty} u_{n} \cos n \theta, v=\frac{a \sigma_{0}}{E} \sum_{n=1}^{\infty} v_{n} \sin n \theta
$$




$$
\begin{aligned}
& w=\frac{a \sigma_{0}}{E} \sum_{n=0}^{\infty} w_{n} \cos n \theta, M_{\xi}=\frac{\sigma_{0} h^{3}}{a} \sum_{n=0}^{\infty} M_{\xi n} \cos n \theta \\
& q=\frac{\sigma_{0} h}{a} \sum_{n=0}^{\infty} q_{n} \cos n \theta
\end{aligned}
$$

The coefficients $\alpha_{1}, \ldots, \alpha_{21}$ are listed in Table A-1 of appendix A. C is a dimensionless damping coefficient defined in the nomenclature.

The following quantities must be prescribed at the bounsaries, $\xi=0,5 / a$.

$$
\left.\begin{array}{c}
N_{\xi} \text { or } u \\
\hat{N}_{\xi \theta}=\left[\bar{N}_{\xi \theta}+\frac{3}{2 a} \bar{M}_{\xi \theta}\right] \text { or } v \\
\hat{Q}_{\xi}=\left[\frac{\partial M_{\xi}}{\partial \xi}+2 \frac{\partial \bar{M}_{\xi \theta}}{\partial \theta}\right] \text { or } w \\
M_{\xi} \text { or } w^{\prime}
\end{array}\right\}=\text { il at } \xi=0, s / a
$$

\section{Fluid Equations}

The fluid both iliside the shell and in the annular region outside the shell will be treated as inviscid $[5,14,15]$, incompressible, and having no gross motion $[3,16,17]$. In other words, the fluid adds inertia to the shell, couples the shell to neighboring structures, and acts as a vehicle for transmitting forces to the shell. As a result, the fluid can be represented by a single equation in terms of one dependent variable, the dynamic pressure $\mathbf{p}$.

Expanding the pressure into a Fourier series about the circumference results in a differential equation with independent variables $\xi, n$, and $\tau$ where $n$ is a dimensionless radial coodinate, $n \equiv r / a$. To couple the fluid 
to the shell, the radial velocities of the shell and fluid are made equal. Also, the assumption is made that the pressure belonging to a given circumferential mode does not excite other than its matching structural mode; an assumption usualiy made in modal analysis $[1,3,5,17]$.

Starting with the equation of motion for inviscid, compressible fluids (Euler's equation), the continuity equation, and assuming small oscillations we obtain the acoustic wave equation [18].

$$
\frac{1}{c^{2}} \frac{\partial^{2} p}{\partial t^{2}}-\nabla^{2} p=0
$$

For an incompressible fluid $(c \rightarrow \infty)$ we may express $\nabla^{2} p$ in $c y l$ indrical coordinates as

$$
\frac{\partial^{2} p_{n}}{\partial r^{2}}+\frac{1}{r} \frac{\partial p_{n}}{\partial r}-\frac{n^{2}}{r^{2}} p_{n}+\frac{\partial^{2} p_{n}}{\partial \xi^{2}}=0,
$$

where we expand $p$ in a Fourier series as

$$
p=\frac{\sigma_{0} h}{a} \sum_{n=0}^{\infty} p_{n} \cos n \theta .
$$

The assumption of incompressibility is valif if the sonic velocity in the fluid is much higher than the velocity of motion of the shell surface and/or the shelf is closely confined in a rigid-walled tank. Both Mnev and and Pertsev [2], and Chen and Rosenberg [3] have shown that compress ibj)ity effects are unimportant in calculating the response of a shell closely confined in a liquid. Hnev and Pertsev providr an analytical solution for a shell with simply-supported end conditions submerged in an acoustic fluid. They show that if the shell is confined, coaxially, inside a rigid-walled outer cylinder, the criteria to determine whether the incompressibility assumption is valid is 


$$
\left[\frac{\Omega b}{c}\right]^{2} \frac{1}{n^{2}+\left(\frac{\pi b m}{5}\right)^{2}} \ll<1 .
$$

For all other cases and when $n=0$

$$
\left[\frac{\Omega s}{\pi m c}\right]^{2} \ll 1
$$

is the criteria to be used. The experimental results reported later substantiate these criteria.

Boundary conditions must be specified on the top and bottom of the fluid regions and at the inner and outer surfaces. Either a pressure release $\left(p_{n}=0\right)$ or rigid wall $\left(\partial p_{n} / \partial \xi=0\right)$ can be specified on the top or bottom of the fluid regions although the fomer more closely matches the analytical solution and experimental results reported here. Similar boundary conditions can be applied to the outer surface of the outer fluid. At the shell surfaces the shell radial velocity must be made equal to the fluid radial velocity. Therefore, using Euler's equation we can express the pressure gradient at the wall as

$$
\frac{\partial p_{n}}{\partial h}= \pm \frac{a}{h} \frac{\rho_{f}}{\rho_{s}} \frac{\partial^{2} w_{n}}{\partial \tau^{2}} \text {. }
$$

To eliminate the singutarity at $n=0$ a rigid inner boundary at $r=r_{0 / a},\left(r_{0} \ll a\right)$ was used.

\section{CALCULATIONAL METHOD}

The basic strategy of the calculational method is to solve the finite difference equations of the shell and fluid separately, but to account for the interaction between the two by imposing the boundary condition (9) at the shell-fluid interface. The shell displacements as a function of time for each Fourier mode are detemined separately and must be summed to get the total displacement. The forcing function, whether it 
is applied on the outer boundary of the fluid or on the shell must be expanded into a Fourier series with the appropriate Fourier tem applied to each mode. The natural frequencies for each mode can be calculated by applying a load that is a step function in time and following the time history of the response. This is the method used in this work. Another means of determining the natural frequencies is to vary the load harmonically and generate a frequency plot.

\section{Shell Equations}

The algorithm used to solve the shell equations is similar to that used by Johnson and Greif [12]. The shell equations of motion (2) for a particular Fourier mode are cast in a matrix form in terms of $z$, a four iélement column vector composed of unknowns $u, v, w, M_{\xi}$,

$$
K z^{\prime \prime}+F z^{\prime}+G z=y+O \ddot{z}+L \dot{z} \text {. }
$$

$K, F, G, D, L$ are $4 \times 4$ coefficient matrices, and $y$ is a column loading vector. A11 are defined in Appendix B. Another matrix equation is developed to describe the shell end conditions,

$$
\hat{\Omega}+z^{\prime}+(I-\Omega+\Omega I) z=\ell,
$$

with $\hat{\Omega}$ a diagonal matrix and $\ell$ a column vector defined by the end conditions. $H$ and $J$ are $4 \times 4$ matrices (see Appendix B) defined by the equations relating Sanders' boundary conditions (4) to z, For example, if $u$ is known at an end, the first diagonal element of $\hat{\Omega}$ is zero, and the first element of $\ell$ is the prescribed value of $u$.

Using difference schemes, equations (10) and (11) are recast into three matrix equations (Appendix B, equations $(B-10 a, b, c$,$) ) in terms of the$ unknown vectors $z_{i+1, j}, z_{i, j}, z_{i-1, j}$ and are solved by a Gaussian elimi- 
nation technique at each time step $j$. Central differencing is used to describe the space derivatives and backward differencing to describe the time derivatives. The backward differencing for the time derivatives (Houbolt method) [19] results in an implicit, unconditionally stable algorithm. To solve the three matrix equations $\bar{x}$ recurrence relationship is used relating $z_{i, j}$ and $z_{i-1, j}$. The coefficients and constants for this relationship are calculated starting at $i=0$ up to $i=N$. Then using the boundary condition at $i=N$ the vectors $z_{N, j}, z_{N-1, j}$, etc. down to $z_{0, j}$ are calculated. This procedure is repeated for successive time steps.

\section{Fluid Equations}

The equation representing the fluid (6) is a form of the Helmholtz equation. The nth Fourier mode of this equation is recast into finite difference form giving an equation for each node (Appendix B, equations $(B-12 a, b, c))$. The resulting set of linear, algebraic equations is solved by a fast, direct solution method [20] which takes advantage of the tridiagonal nature of this set and employes an odd-even cyclic reduction iechnique. The axial dimension must be subdivided into $N>2$ increments that do not include multiples of $7,11,13,17$, etc.

We were interested in simulatiny pump pressuce pulses coming into a pressure vessel and therefore modified the direct solution method so as to handle the specification of mixed boundary yalues on the outer fluid boundary. Thus, a pressure profile, $p(t)$, can be specified over the inlet region and a rigid wall, $\partial p / \partial r=0$, over the remainder of che outer boundary. A capaitance matrix technique $[21,22]$ was used to modify the direct solver so that mixed boundary values could be handled. This involved a modification of the rows of the matrix form of the set of finite differ- 
ence equations such that the cyclic reduction process could still be used on the modified system.

\section{Calculation Technique}

In the original paper by Johnson and Greif [12] constraints were placed on the time and space increments. As the time increment gets large, the calculated shell response appraches the corract static solution but any dynamic response is damped out. The time increment must be kept to less than 1/50 of the period of a particular mode of vibration so that the displacements are not significantly damped. This damping results from the time integration scheme used (Houbolt method) and is strictly a numerical effect. The tendency of the fluid is to increase this numerical damping. The damping increases as the fluid reaction becomes more significant either due to closer shell spacing, higher fluid density or for the lower shell modes. To maintain damping less than $1 \%$ for calculations involving a fluid, time steps between 1/50 and $1 / 500$ of the period of vibration were used.

The space increment must be kept small enough to cescribe adequately the axial bending moment, $M_{E}$, which is proportional to the second time derivative of the radial displacement. In general, 120 axial nodes were found to give adequately resolved displacements and bending moments.

As previously stated, the original shell code is unconditionally stable. It was found, however, that the addition of the fluid resulted in an unstable solution when the hydraulic loading exceeded trice the inertial force generated in the shell. This numerical instability is believed to be caused by the weak coupling employed to connect the shell and fluid equations. Belytschko [9] has reported on numerical inctabilities 
in fluid-structure codes from this cause. Weak coupling results when two separate programs are used to form a complete solution. In our case, this weak coupling was through the boundary condition (9) at the shel1fluid interface. We made several attempts to circumvent this instability, i.e., incrementing down the shell density, incrementing up the fluid density, iterating on the pressure at each time step, all without success. To owercome this instability, the shell and fluid equations would have to be more strongly coupled, i.e., made more interdependant. This would require a complete reformulation of these equations or a change to an explicit type of time integration and incorporation of several boundary nodes in both the she11 and fluid equations.

The stability criterion that was found may be stated as

$$
\frac{\text { hydraulic resisting pressure }}{\text { sheTl iriertial force per unit area }}=\rho_{f} \Phi / \rho_{s} h<2
$$

where $\Phi$ is called the connected mass factor. In general $\Phi$ is a function of mode number and geometry and increases as the mode numbir or gap between sheli and outer fluid boundary decreases.

For those cases where the stability criterion cannot be net, an approximate solution can be computed by using an increased shell density and decreased fluid density so that the effective mass, $M_{e f f}$, is held constant.

$$
\begin{aligned}
M_{\text {eff }} & =\rho_{s} h+\rho_{f} \Phi \\
\Phi & =-p(a) / \rho_{f} \partial^{2} w / \partial t^{2}
\end{aligned}
$$

The connected mass factor, $\Phi$, is computed according to (14) by using in the computation a normal shell density, $p_{5}$, and a decreased fluid density, 
$\rho_{f}$, such that the inequality of (12) is satisfied. The validity of this technique has been established by Mnev and Pertsev [2] who showed, that for the range of problems for which the incompressibility assumption is valid, $(7,8)$ the effect of the fluid can be adequately described by i'screasing the shell surface density by the amount $\rho_{f} \Phi$. The connected moss factor is a function of the axial mode excited, $m$, as well as the circumferential mode $n$. Therefore, the accuracy of $\Phi$, riputed is closest when only a single axial mode is excited. Fortunately, for most loadings, the fundamental is predominant. Also for the higher modes $(n>2)$ the difference in $\Phi$ between axial modes is $10 \%$ or ?ess. For a particular mode, $\Phi$ can be calculated as closely as desircd by applying, at the modes anti-node, a harmonic load close to the mode's natural frequency.

\section{EXPERIMENTAL VERIFICATION}

An experimental program was undertaken to verify the numerical technique. A thin walled, circular shell was fabricated and instrumented with two dynamic pressure transducers and eight miniature accelerometers. These read the pressure or the shel $1^{\text {t's }}$ outer surface and its radial acceleration. The experimental arrangement is shown in Fig. 2 and approximates a nuclear pressurized water reactor core barrel at 1/12 scale.

The natural frequencies and radial accelerations of the shell were measured in air, water, and oil. Excitation with liquid was provided by a piston-cylinder arrangement connected to an electro-magnetic exciter. Sinusoidal pressure waves were generated in the cylinder and conducted to two inlet ports, finally impinging on the shell. A frequency sweep identified the shell resonant frequencies and modes from the accelerometer signals. In addition, the natural frequencies were measured using an impact technique [23]. 

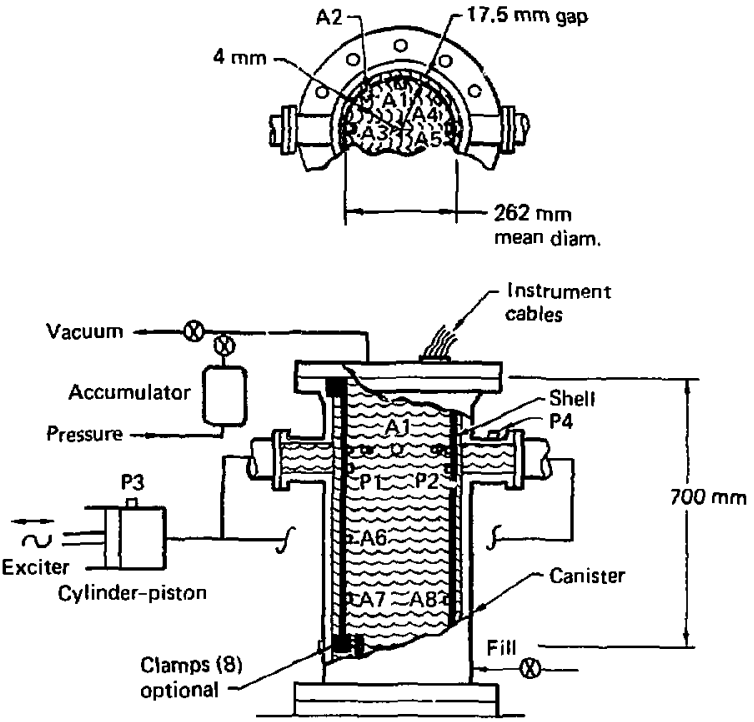

Instrumentation

Accelerometers, $A 1$ through $A B$

Pressure transofucers, P1 through P4

Fig. 2 Experimental contigration. 
Measured and numerical results in air, water, and oil are shown in Tables la and $1 \mathrm{~b}$.

For a simply-supported shell in water, connected mass factors were calculated in accordance with Mnev and Pertsev [2] and compared to numerical results (14). This comparison is shown in the third and fourth columns of Table 1a. In the remaining columns comparison is made between mecsured and calculated natural frequencies for each mode. The measured results for air were obtained using the impact technique and for water using both the impact technique and hamonic excitation, with the results averaged. The numerical calculations were made witn the shell modeled assuming it fixed at the top end and either simply-supported or free at the bottom: end. The ends of the liquid region were held at constant pressure and the outer fluid boundary assumed rigid. Measured and calculated natural frequericies in oil are compared in Table 1b. Natural frequencies in oil were measured using harmonic excitation.

Figs. 3a and $3 b$ show the natural frequencies of the experimental shell as a function of circumferential mode number for fixec-simplysupported and fixed-free end conditions. The solid and dottes ines trace through the numerical results for air and water, respectively and the symbols indicate measured data. Open symbols denote impact results in air, solid, impact results in water, and crossed, harmonic results in water. Air and water results for a given shell end condition are shown on the same figure for the first three axial modes.

As noted by Kraus [24] and others, the lowest natural frequency of thin shells is not at the lowest circumferential mode number and in our case is at $n=2$ or 3 . Frequency should and does increase with increasing axial mode number, $m$. The decrease in natural frequency due to the water is quite dramatic as would be expected for the confined 
Table 1a - Comporison of Colculated and Measured Shell Natural Frequencles in Air and Mater

\begin{tabular}{|c|c|c|c|c|c|c|c|c|c|c|c|c|c|}
\hline $\mathbf{n}$ & \multicolumn{3}{|c|}{$\begin{array}{l}\text { Shel1 SImply- } \\
\text { Supported (both ends) } \\
\qquad n=y=H_{E}=N_{E}=0\end{array}$} & \multicolumn{5}{|c|}{$\begin{array}{l}\text { Shel1 Fixed-51mply-Supported } \\
u=v=*=w^{\prime}=0, v=w=H_{E}=N_{E}=0\end{array}$} & \multicolumn{5}{|c|}{$\begin{array}{l}\text { Shell Fixed-Free } \\
=H^{\prime}=0, N_{E}=\hat{N}_{E \theta}=\hat{Q}_{E}=H_{E}=0\end{array}$} \\
\hline & $m$ & \multicolumn{2}{|c|}{$\begin{array}{l}\text { Connected Mass } \\
\text { Factor, } \Phi(m)\end{array}$} & $m$ & \multicolumn{2}{|c|}{$\begin{array}{l}\text { Hatural Fraquency } \\
\text { in AIr, } \omega / 2 \pi(\mathrm{Hz})\end{array}$} & \multicolumn{2}{|c|}{$\begin{array}{l}\text { Natural Frequency } \\
\text { In } \mathrm{Hatar}, \\
n / 2 \pi(\mathrm{Hz})\end{array}$} & \multicolumn{3}{|c|}{$\begin{array}{l}\text { Natural Frequency } \\
\text { In } \mathrm{Atr}, \omega / 2 \pi(\mathrm{Hz})\end{array}$} & \multicolumn{2}{|c|}{$\begin{array}{l}\text { Natural Frequency } \\
\text { in Hater, } \\
\Omega / 2 \pi(\mathrm{Hz})\end{array}$} \\
\hline & & Ansilytic & Numerical & & Measured & Numerical & Measured & Nunerical & & Measured & Numertcal & Measured & Hismertcal \\
\hline 1 & 1 & 0.79 & 0.78 & & & & & & & & & & \\
\hline 2 & 1 & 0.28 & 0.28 & 1 & 530 & 640 & 200 & 205 & $\begin{array}{l}1 / 2 \\
3 / 2\end{array}$ & $\begin{array}{l}200 \\
740\end{array}$ & $\begin{array}{l}230 \\
860\end{array}$ & $\begin{array}{r}75 \\
315\end{array}$ & $\begin{array}{r}75 \\
300\end{array}$ \\
\hline 3 & 1 & 0.14 & 0.15 & $\begin{array}{l}1 \\
2\end{array}$ & $\begin{array}{c}550 \\
525,1005\end{array}$ & 555 & $\begin{array}{l}245 \\
475\end{array}$ & $\begin{array}{l}235 \\
540\end{array}$ & $\begin{array}{l}1 / 2 \\
3 / 2 \\
5 / 2\end{array}$ & $\begin{array}{r}440 \\
620 \\
1150\end{array}$ & $\begin{array}{r}435 \\
640 \\
1220\end{array}$ & $\begin{array}{l}195 \\
300 \\
625\end{array}$ & $\begin{array}{l}185 \\
275 \\
309\end{array}$ \\
\hline 4 & 1 & 0.094 & 0.094 & $\begin{array}{l}7 \\
2 \\
3\end{array}$ & $\begin{array}{r}B 60 \\
1050 \\
1440\end{array}$ & $\begin{array}{r}860 \\
1170 \\
1470\end{array}$ & $\begin{array}{l}440 \\
580 \\
860\end{array}$ & $\begin{array}{l}430 \\
600 \\
760\end{array}$ & $\begin{array}{l}1 / 2 \\
3 / 2 \\
5 / 2\end{array}$ & $\begin{array}{l}820 \\
890\end{array}$ & $\begin{array}{l}820 \\
880\end{array}$ & $\begin{array}{l}420 \\
480 \\
645\end{array}$ & $\begin{array}{l}410 \\
450 \\
575\end{array}$ \\
\hline 5 & 1 & 0.066 & 0.069 & $\begin{array}{l}1 \\
2\end{array}$ & $\begin{array}{l}1350 \\
1460\end{array}$ & $\begin{array}{l}1345 \\
1485\end{array}$ & $\begin{array}{l}770 \\
860\end{array}$ & $\begin{array}{l}750 \\
850\end{array}$ & $\begin{array}{l}1 / 2 \\
3 / 2\end{array}$ & $\begin{array}{l}1325 \\
1375\end{array}$ & $\begin{array}{l}1310 \\
1350\end{array}$ & $\begin{array}{l}755 \\
805\end{array}$ & $\begin{array}{l}750 \\
755\end{array}$ \\
\hline
\end{tabular}

Table Ib - Comparison of Calculated and Hessured Shell Hatural Frequencles in 011

\begin{tabular}{|c|c|c|c|c|c|c|}
\hline$n$ & \multicolumn{3}{|c|}{ Sheli Fixed-Sinply-Supported } & \multicolumn{3}{|c|}{ Shelt Flxed-Free } \\
\hline & $\mathrm{m}$ & Hatural Frequency in $011, \Omega / 2$ & (Hz) & in & Hotural frequency in 011,5 & $\sqrt{2 \pi(\mathrm{Hz})}$ \\
\hline & & \begin{tabular}{l|l} 
Measured \\
\end{tabular} & Nirmerical & & Measurad & Finerias \\
\hline 3 & 1 & 240 & 245 & $1 / 2$ & $2 \mathrm{Cs}$ & 195 \\
\hline 4 & J & 450 & 450 & $1 / 2$ & 435 & 430 \\
\hline 5 & 1 & 795 & 780 & $1 / 2$ & 780 & 780 \\
\hline
\end{tabular}




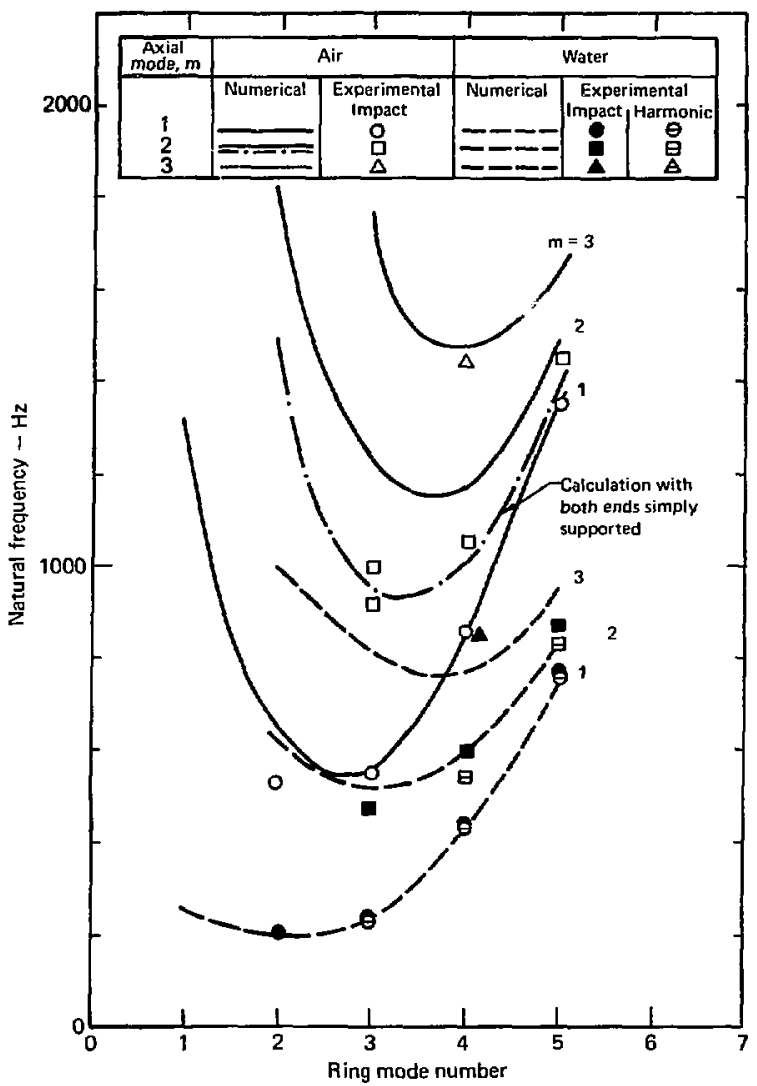

Fig. 3a Natural frequencies of model core barrel with fixed-simply supported end conditions. 


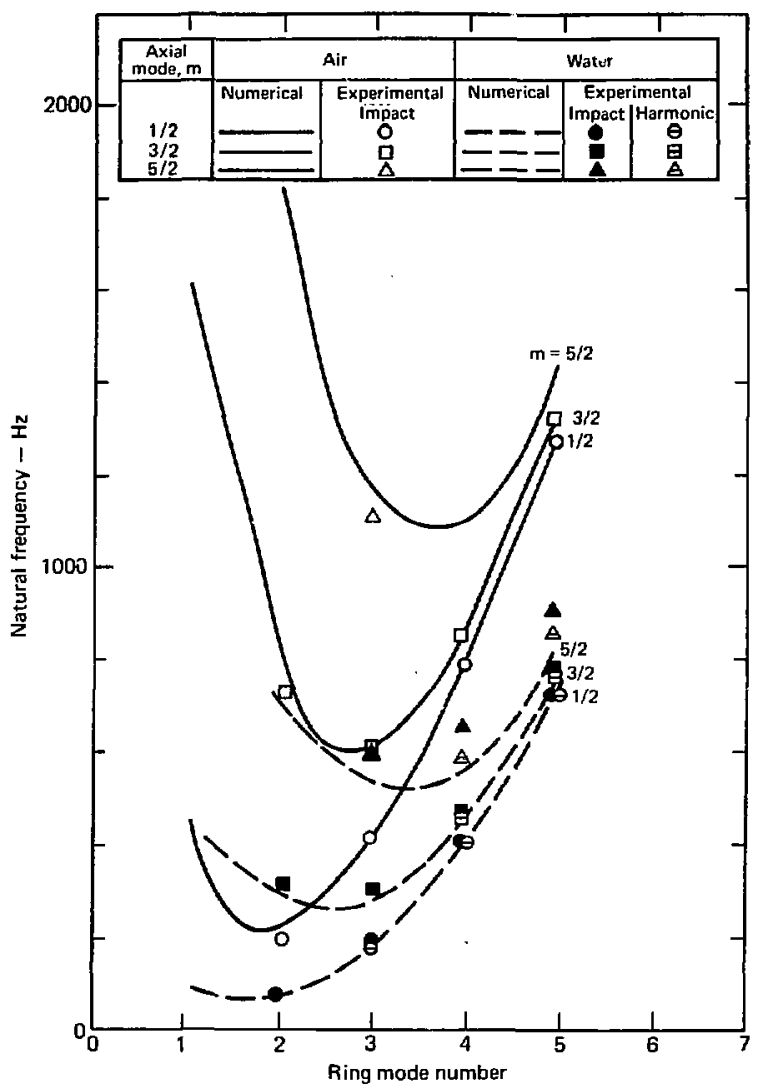

Fig. 3b Natural frequencies of model core barrel with fixed-free end conditions. 
geometry of the experiment. Note that in Fig. 3a (see dot-dash line) the numerical and experimental results in air for te $\mathrm{m}=2$ mode compare better when simply-supported end conditions are specified at both ends in the calculation instead of fixed at one end and simply-supported at the other. For the other cases of Fig. 3a, numerical calculations using fixed-simply-supported shell end conditions compare best with the measured frequencies. Also note that two natural frequencies were measured for the $(3,2)$ mode in air. Both anomalies are probably caused by the method used to support the experimental shell.

Details of a numerical calculation of the experimental shell's nature frequencies for the $n=2$ and $n=5$ modes are shown in Figs. $4 a$ and 4b. The use of the connected mass factor is illustrated by these calculations.

The shell is assumed immersed in water in a rigid-walled container with pressure held constant at the end of the fluid region. The ends of the shell are fixed-simply-supported and an impulse load is applied at the shell's middle. The figures show the time history of the radial displacement three-quarters from the fixed end. The solid line shows the computer response for zero fluid density with the shell density determined using the effective mass calculated according to (34). The dotted lines are calculations using the same effective mass but a fluid density corresponding to the maximum allowed by (12). For the $n=2$ modes (Fig. 4a) this maximum allowed density is $720 \mathrm{~kg} / \mathrm{m}^{3}$ and for $n=5$ (Fig. 4b) it is the normal density of water, $1000 \mathrm{~kg} / \mathrm{m}^{3}$.

In Fig. 4a a more accurate determination of the connected mass factor, $\$$, would have decreased the small (5\%) discrepancy in frequency. In Fig. 4b, itie effect of numerical damping when a finite density fluid is used in the calculation is evident. The calculated frequencies are within $5 \%$ of the 

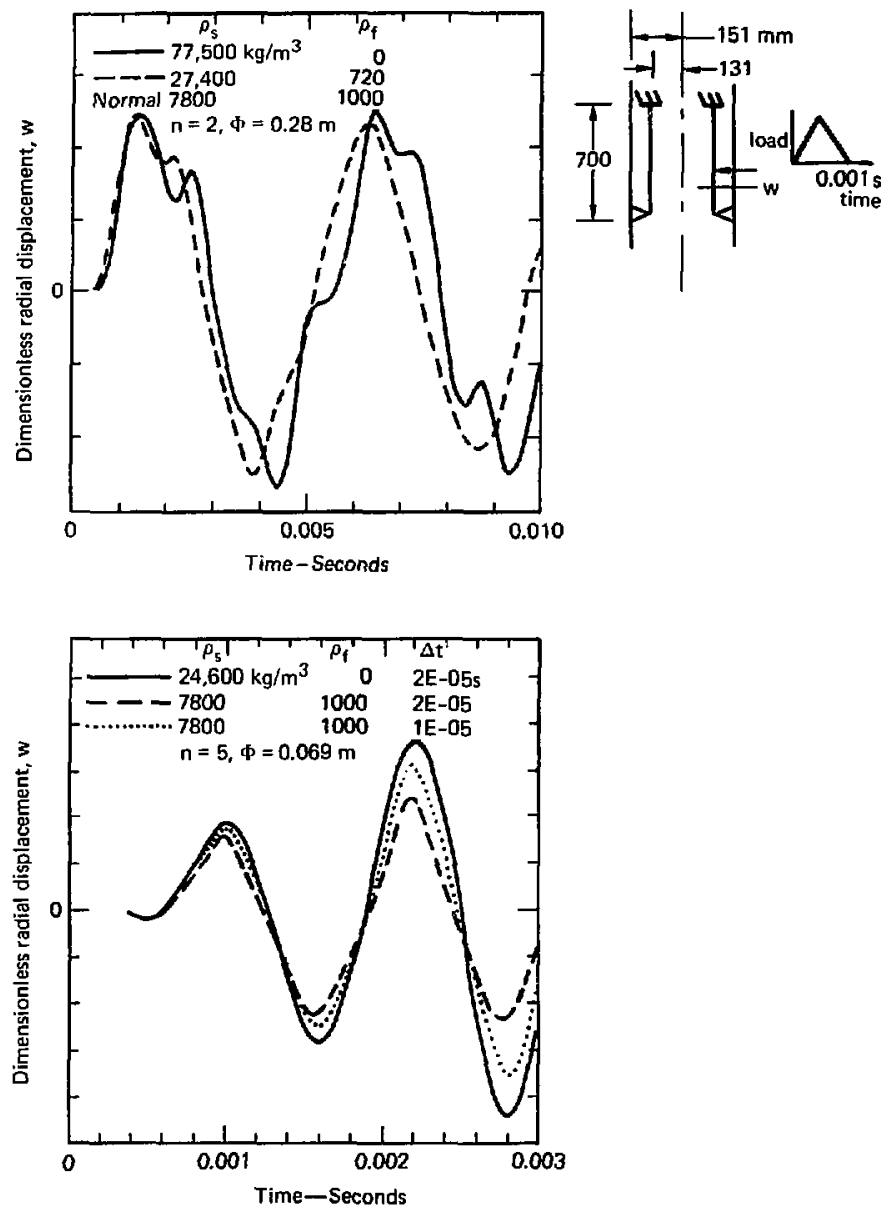

Fig. 4 Calculation of natural frequency using the connected mass factor. 
measured frequencies in water of $200 \mathrm{~Hz}(n=2)$ and $770 \mathrm{~Hz}(\mathrm{n}=5)$ respectively.

Care was taken to make sure that the experiment would praduce results which cauld provide a valid comparison to numerical results. Incompressible fluids (deaerated water and oil) and an elastic (stainless steel) shell material were used. The geometry was such that the potentiai fluid approximation was valid according to equation (7). Two different shell end conditions and two nozzle arrangements were used. Measurements of the damping coefficients proved them low enough $(<1 \%)$ to be modeled in the simple manner that they were, i.e., as simple viscous structural damping coefficients for each mode. The fact that $t$ - natural frequencies measured match closely those predicted by calculaticn speak for the validity of the experiment.

Referring to Tables $1 \mathrm{a}$ and $1 \mathrm{~b}$ it can be seen that the measured natural frequencies match those calculated within $5 \%$ in three-quarters of the cases. The maximum discrepancy is $17 \%$ for the $n=2, m=1$, mode in air. Since this error applies to the air results it appears the major part of all errors are due to the well know difficulty of modeling end conditions in an experiment. Also note that the numerically calculated connected mass factor matches the analytical result within 5\% (Table 1a).

\section{APPLICATION OF NUMERICAL TECHNIQUE}

To illustrate how the numerical technique that has been described can be used to calculate natural frequencies and connected mass factors we calculate these for the core barrel of a pressurized water reactor. We compare these numerical solutions to analytical solutions of Horvay and Bowers [17] which restricts us to specifying simply-supported end conditions. The modes of interest for this problem are the cantilever mode i $n=\uparrow$, 
$m=1 / 2)$ and the fundamentals of the first few ring modes $(n=2, \ldots, 6$, $\mathrm{m}=1)$ [25].

The shell is filled with air and surrounded by a thin, fluid annulus of either air or water enclosed by a rigid outer wall. The dynamic pressure, $p$ is held constant at the top and bottom. With reference to Fig. 1 the important parameters are:

$$
\begin{aligned}
a & =1.92 \mathrm{~m}(76 \mathrm{in.}) & \rho_{f} & =1.2 \mathrm{~kg} / \mathrm{m}^{3} \text { (air) } \\
b & =2.18 \mathrm{~m} \text { (86 in.) } & & =1000 \mathrm{~kg} / \mathrm{m}^{3} \text { (water) } \\
\mathrm{h} & =0.051 \mathrm{~m}(2 \mathrm{in.}) & \rho_{\mathrm{s}} & =7800 \mathrm{~kg} / \mathrm{m}^{3} \\
\mathrm{~s} & =12.91 \mathrm{~m}(328 \mathrm{in.}) & \mathrm{E} & =2.1 \times 10^{-11} \mathrm{~N} / \mathrm{m}^{2}\left(30 \times 10^{6} \mathrm{psi}\right)
\end{aligned}
$$

The results of these calculations and the comparison with the analytical results are shown in Table 2. The natural frequencies in air were calculated using normal air density. This gives the same frequency as if calculated in a vacuum with the additional benefit of providing a connected mass factor for later use. Comparisons between numerical and analytical results in air were good with a maximum discrepancy of 13\%. The frequency for the $n=1, m=1 / 2$ mode was calculated assuming a simplysupported shell of twice the length and also by assuming shell of nominal length but fixed at one end. Calculations by the first method gave a natural frequency $23 \%$ lower than calculations by the second method. No analytical results were available for comparison to the fixed-free calculation.

The connected mass factors and natural frequencies with the shell filled with air and surrounded by water are shown in the last four columns of Table 2. For the $n=5,6$ modes nomal shell and fluid densities were used 
Tahle 2 - Pressurized Hater Reactor Core Barrel Naturai Frequencies

\begin{tabular}{|c|c|c|c|c|c|c|c|c|}
\hline \multirow{3}{*}{$n$} & \multirow{3}{*}{$\mathrm{m}$} & \multirow{3}{*}{$\begin{array}{l}\text { End- } \\
\text { Con- } \\
\text { ditions }\end{array}$} & \multirow{2}{*}{\multicolumn{2}{|c|}{ Air }} & \multicolumn{4}{|c|}{ Water } \\
\hline & & & & & \multicolumn{2}{|c|}{$\Phi(m)$} & \multicolumn{2}{|c|}{ Frequency $\left(H_{x}\right)$} \\
\hline & & & $\begin{array}{l}\text { Present } \\
\text { Methnd }\end{array}$ & Ref, 17 & $\begin{array}{l}\text { Present } \\
\text { Method }\end{array}$ & Ref. 17 & $\begin{array}{l}\text { Present } \\
\text { Methad }\end{array}$ & Ref. 17 \\
\hline 1 & $1 / 2$ & A & 33 & 35.7 & 13.6 & 7.19 & $6-7$ & 8.18 \\
\hline 1 & $1 / 2$ & B & 43 & & $\begin{array}{c}9.5 \\
11.5 *\end{array}$ & & $\begin{array}{c}8.5-11 \\
8-10^{\star}\end{array}$ & \\
\hline 1 & 1 & $C$ & 105 & 106.4 & $\stackrel{9.5}{11.5 *}$ & 5.28 & $\begin{array}{l}21-25 \\
19-23\end{array}$ & $26=$ \\
\hline 2 & 1 & C & 45 & 47.7 & 3.5 & 3.66 & $14.5-15.5$ & 15.0 \\
\hline 3 & 1 & C & 35 & 38.4 & 1.7 & 1.80 & 16 & 16.4 \\
\hline 4 & 1 & $\mathrm{C}$ & 53 & 55.9 & 1.02 & 1.07 & 28 & 29.2 \\
\hline 5 & 1 & C & 82 & 85.0 & 0.60 & 0.71 & 50 & 50.9 \\
\hline 6 & 1 & $c$ & 140 & 121.8 & 0.45 & 0.51 & 80 & 80.5 \\
\hline
\end{tabular}

She11 End Conditions:

A - Shell simply-supported at both ends but twice as long

B - She11 fixed at one end and free at the other

C - Shell simply-supported at both ends

*Shell filled and surrounded by water. 
in the numerical calculation, making the use of their connected mass factors unnecescary. For lower modes, the shell density was increased and the fluid density lecreased holding $M_{\text {eff }}$ constant so that a stable solution could be obtained. For all but the beam modes $(n=1)$ comparjson between numerically and analytically calculated mass factors and frequencies wa.s within $12 \%$.

We assumed that holding the effective mass constant would assure a correct calculation of the natural frequencies no matter what specific values are used for the shell and fluid densities. To check this assumption we increased the shell density and decreased the fluid density while holding the effective mass constant and compared the natural frequencies so calculated. We found, for instance, that for the $n=1, m=1$ mode the frequency decreased from $25 \mathrm{~Hz}$ to $21 \mathrm{~Hz}$ (see Table 2) as $\rho_{s}$ was increased form $8.3 \times$ $10^{4} \mathrm{~kg} / \mathrm{m}^{3}$ to $240 \times 10^{4} \mathrm{~kg} / \mathrm{m}^{3}$ and the corresponding fluid density decreased from $690 \mathrm{~kg} / \mathrm{m}^{3}$ to 0 . For the $n=2$ mode a $1 \mathrm{hz}$ difference was found when the densities were changed and for $n=3,4$ no difference. These frequency differences reflect the accuracy of our numerical technique.

Natural frequencies were also calculated with the shell filled and surrounded by water for the cantilever and first beam mode. As expected, ho connected mass factors increased and the frequencies decreased as compared to results for the shell filled with air and surrounded by water. For the beam modes, only half of the fluid component of tive effective mass was used in the analytic calculations. This was a method used by Horvay and Bowers to make their anaiytical solution conform to Etokes' formula for solid body motion. Our results indicate that the entire effective mass should be used for all modes, Including the beam modes, and hence our numerically calculated frequencies are lower than theirs for the beam modes. 
Running times for this problem on a CDC 7600 varied according to whether fluid was present or not. With no fluid a 300 time step, 120 space station run took $0.36 \mathrm{~min}$. With fluid only outside the shell it took $0.93 \mathrm{~min}$. With fluid both inside and outside, it took $1.70 \mathrm{~min}$.

CONCLUSION

We have presented a numerical solution technique which can be used to determine the naturat frequencies and transient responses of thin cylindrical shel1s imiersed in an incompressible, inviscid fluid.

The shell may be filled with fluid, surrounded by fluid, both of these, or immersed in a fluid-filled, stiff-walled coaxial container. Arbitrary radial loads can be applied directly to the shell or over a segment of the outer fluid boundary. Any of the natural boundary conditions can be applied to the ends of the shell. The ends of the fluid regions can be either open or closed.

Direct solution of problems where the ratio of the hydrodynamic forces to the shell inertial forces are greater than two is not possibie because of numerical instability. In this regime, an effective mass is calculated to be included as increased shell density, circumventing the numerical instability. This numerical instability is bel ieved to result from the weak coupling between the equations describing the shell and fluid. This weak coupling is represented by the boundary condition equation (9) and is the result of using two separate programs to represent the fluid and shell. Future efforts to remove this instability should be directed toward strong coupling of the equations which will require their reformulation if one desires a fully implicit time integration scheme. An implicit scheme is felt to be necessary to gain steady-state solutions in a reasonable time. 
Although solutions to the fluid-shell problem are possible using analytical techniques, only simply-supported shell end conditions have been specified using this approach. Our numerical technique allows specification of any of the natural shell end conditions. Also the analytical solution for a narrow water annulus [17] implies that only one-half the calculated effective mass should be employed to calculate the shell response in the beam mode $(n=1)$. By using our numerical technique, we were able to show that the full effective mass should be used.

Rapid solutions using the numerical technique described here are possible making feasible, design sensitivity studies for problems where resonant conditions are a concern. By use of this technique, placement of pump inlet ports, shell thickness, end conditions, and annular spacings can be established to circumvent resonant conditions and to minimize displacements and stresses.

The most significant contribution from this study is that it provides a verified technique for determining the natural frequencies, modes, effective masses, and displacements of a cylindrical shell immersed in a potential fluid. Other theories either do not oirectly couple the shell to the fluid, cannot treat the annular geometry, calculate only an r.m.s. displacement, or can only calculate the transient response of a shell to a sudden load such as from a reactor accident. 
NOMENCLATURE

$A, B, C=4 \times 4$ matrices $(B-10)$

$\hat{A}, \hat{B}, \hat{C}=$ block tridiagonal and capacitance matrices $(B-14,15,17)$

$a=c y l$ inder radius

$b=$ outer annular radius

$C=$ dimensioniess damping coefficient $=\frac{\lambda a}{\left[(E)(h)\left(\rho_{s} h+\rho_{f} \phi\right)\right]^{1 / 2}}$

$c_{c}=$ critical damping coefficient $=2 M M_{\text {eff }} \Omega$

$c=$ acoustic velocity of fluid

$D=4 \times 4$ matrix $(10)$

$D=\frac{h^{3} E}{12\left(1-v^{2}\right)}$

$E=4 \times 4$ matrix $(B-10)$

$E=$ modulus of elasticity

$e_{\xi}, \epsilon_{\theta}=$ strains at an arbitrary point in the shell

$F=4 \times 4$ matrix (10)

$F_{1}, F_{2}, F_{4}$, functionals

$G=4 \times 4$ matrix $(70)$

$H=4 \times 4$ matrix (11) 


$$
\begin{aligned}
& h=c y l i n d e r \text { thickness } \\
& I=\text { identity matrix } \\
& J=4 \times 4 \text { matrix (11) } \\
& K=4 \times 4 \text { matrix }(10) \\
& K=\frac{h E}{\left(T-v^{2}\right)} \\
& L=4 \times 4 \text { matrix }(10) \\
& L_{i j}=\text { linear operators for shell equations of motion (A-33a,b,c) } \\
& \ell=1 \times 4 \text { column matrix (11) } \\
& M_{\text {eff }}=\text { effective mass (13) } \\
& M_{\xi}, N_{\theta}, H_{\xi \theta}=\text { moment resultants per unit length } \\
& \bar{N}_{E \theta}=\text { moment resultant per unit length, } 1 / 2\left(M_{\xi \theta}+M_{\theta E}\right) \\
& m \text { axial half wave number } \\
& N_{\xi}, N_{\theta}, N_{\theta \xi}=\text { force resultants per unit length } \\
& \bar{N}_{\xi \theta}=\text { force resuitant per unit length, } 1 / 2\left(N_{\xi \theta}+N_{\theta \xi}\right)+1 / 4 a\left(M_{\xi \theta}-M_{\theta \xi}\right) \\
& \hat{N}_{\xi \theta}=\text { effective membrane shear resultant per unit length (4) } \\
& \text { n = circumferential mode number } \\
& P=4 \times 4 \text { matrix (B-11a) } \\
& P_{t}, p_{0}=\text { total and static fluid pressures }
\end{aligned}
$$




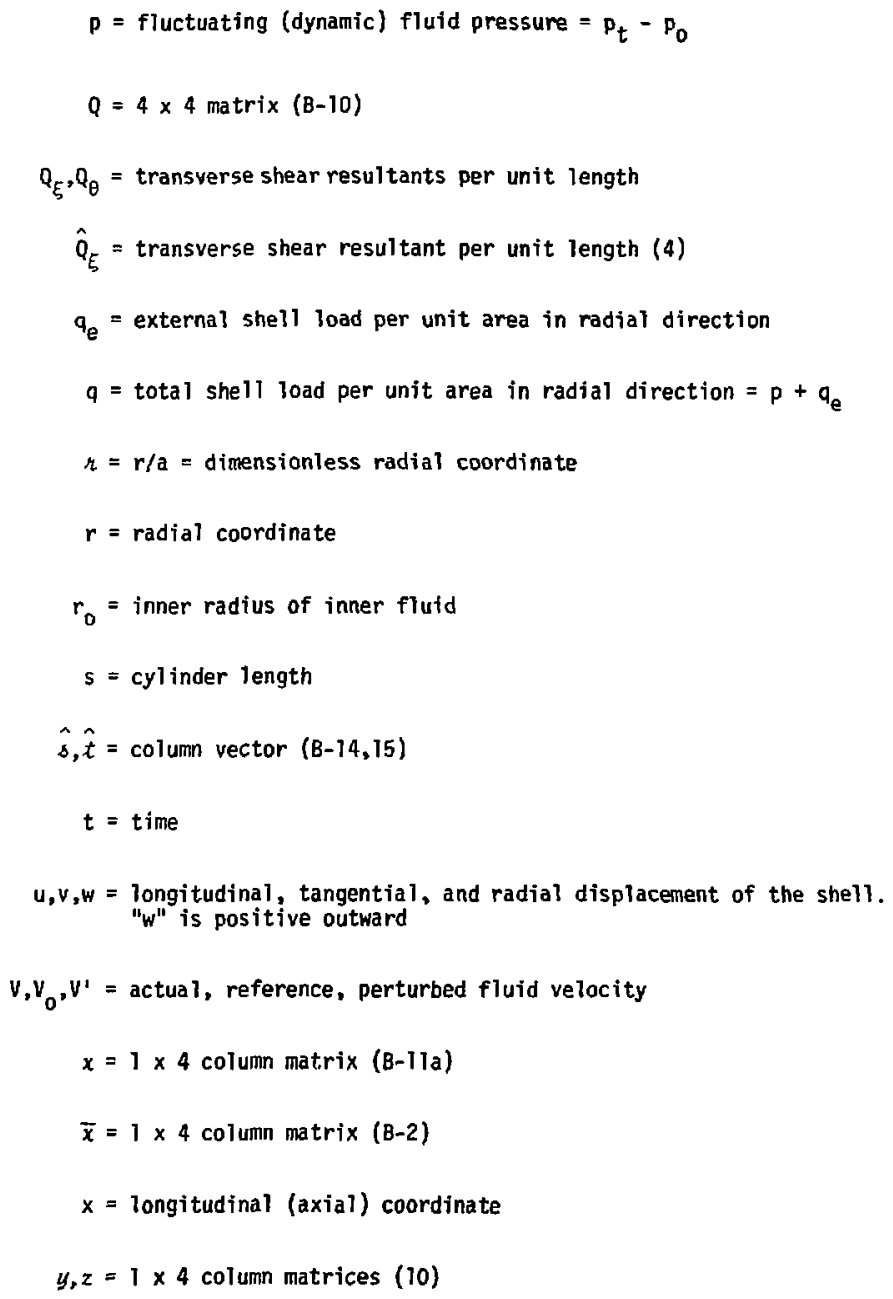


$\alpha_{1}, \alpha_{2}, \ldots, \alpha_{21}=$ coefficients for shell equations of motion (2)

$\bar{\alpha}_{j}=$ coefficient for time derivative equation (B-6a)

$B_{1}, \beta_{2}, \ldots, \beta_{10}=$ coefficients for shell boundary condition $(A-21)$

$\bar{B}_{j}=$ coefficient for time derivative equation ( $B-6 a$ )

$\gamma_{\xi}, \gamma_{\theta}, \gamma_{\xi r}, \gamma_{\theta r}=$ shear strains

$\bar{\gamma}_{j}=$ coefficient for time derivative equation (B-6a)

$\Delta=$ dimensionless axial space increment $=\mathrm{s} / \mathrm{aN}$

$\bar{\delta}_{j}=$ coefficient for time derivative equation (B-6a)

$\varepsilon=$ dimensionless time increment $=\left(E / \rho_{s}\right)^{1 / 2}\left(t_{i, j+1}-t_{i, j}\right) / a$

$\varepsilon_{\xi}, \varepsilon_{\theta}, \varepsilon_{\xi \theta}=$ norma $]$ and shear strains in the shell midsurface

$\zeta=$ compressibility factor $(A-38 C)$

$\theta_{\xi}, \theta_{\theta}, \theta_{r}=$ rotations

$\theta=$ circumferential angular coordinate

$K_{\xi}, K_{\theta}, K_{\xi \theta}=$ changes in curvaicure of the she 11 midsurface

$\bar{k}_{j}=$ coefficient for time derivative equation $(B-6 b)$

$\Lambda=4$ component diagonal matrix $(B-2)$

$\lambda=$ viscous demping coefficient

$\bar{\lambda}_{j}=$ coefficient for time derivative equation $(B-6 b)$

$\bar{\nu}_{j}=$ coefficient for time derivative equation (B-6b) 


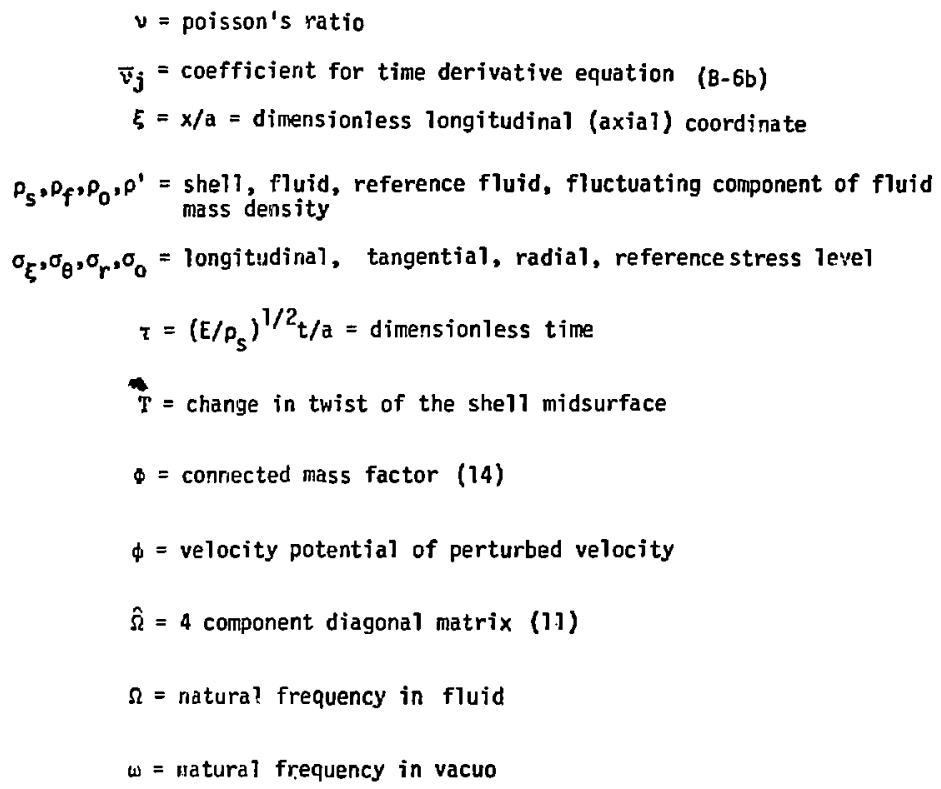

ACKNOWLEDGMENTS

Messrs. W. Arnold, V. Karpenko, G. Broadman, and C. Walter of the Lawrence Live mbre Laboratory authorized and supported this research. Professors H. 8randt, H. A. Dwyer, and D. C. Kamopp of the University of California at Davis gave technical guidance throughout the project. 


\section{REFERENCES}

1. Bleich, H. and Baron, M., "Free and Forced Vibrations of an Infinitely Long Cylindrical Shell in an Infinite Acoustic Medium," J. Applied Mechanics, Vol. 76, (1954), pp. 167-177.

2. Mnev, Y. and Pertser, A., "Hydro Elasticity of She11s," Foreign Technology Division, Wright-Patterson Air Force Base, Ohio. Rpt FTD-MT-24-119-71, (Aug. 1971).

3. Chen, S. and Rosenberg, G., "Dynamics of A Coupled Shell-Fluid System," Nuc. Eng. \& Des., Vol. 32, (1975), pp. 302-310.

4. Bowers, G. and Horvay, G., "Forced Vibrations of a Shell Inside of a Narrow Water Annulus," Nuc. Eng. and Des., Vol. 34, (1975), pp. 221-231.

5. Au-Yang, M. K., "Response of Reactor Internals to Fluctuating Pressure Forces," Nuc. Eng. and Design, vol. 35, (1975), pp. 361-375.

6. Magrad, E. and Burroughs, C., "Forced Harmonic and Random Vibrations of Concentric Cylindrical Shells Immersed in Acoustic Fluids," J. Acoustic Soc. of Am., Vol. 52, (3), Pt. 2, (1971), pp. 858-864.

7. Au-Yang, M. K. and Connelly, W. H., "A Computerized Method for Flow-Induced Randon Vibration Analys is of Nuclear Reactor Internals," Nuc. Eng. and Design, Vol. 42, (1977), pp. 257-263.

8. Berger, B. S. and Palmer, M. E., "The Transient Motion of a Cylindrical Shell of Arbitrary Cross Section in an Acoustic Medium," J. Applied Mech., (Sept. 1977), pp. 482-486.

9. Beiytschko, T., "Method and Programs for Analysis of FluidStructure Systems," Nuc. Eng. and Design, Vol. 42, (1977), pp. 41-52.

10. Sanders, J. L., Jr., "An Improved First-Approximation Theory for Thin Shells," NASA Rept. 24, (1959).

11. Budiansky, B. and Radkowski, P., "Numerical Analysis of Unsymmetrical Bending of Shells of Revolution," AIAA Journa 1, Vol. 1, No. 8, (1963), Pp. 1833-1844.

12. Johnson, D. E. and Greif, R., "Dynamic Response of a Cylindrical Shel 1: Two Numerical Methods," AIAA Journa 1, 4, (3), (1966), pp. 486-494.

13. Leissa, A. W., Vibration of Shel1s, National Aeronautics and Space Administration Report KASA Sp-288, (1973).

14. Lazzeri, L. and Dini, D., "Vibrations of the Internals of Pressurized Hater Reactors: "Proc. of the First Inter. Conf. on Structural Mechanics in Reactor Technology, Vol. 3, (1971), Paper E/8. 
15. Fritz, R., "The Effect of Liquids on the Dynamic Motions of Immersed Solids," Journal of Engineering for Industry, Vol. 94, (Feb. 1972), pp. $167-173$.

16. Lazzeri, L. and Dini, D., "Vibrations of a Pendular Structure of Circular Cross-Section Inside a Concentric Pipe," Ingegneria Nucleare, Vol. 10, (4), (1969), pp. 47-58.

17. Horvay, G. and Bowers, G., "Influence of Entrained Water Mass on the Vibration Modes of a She11," J. of Fluids Engineering, (June 1975), pp. 211-216.

18. Landeau, L. D, and Lifshitz, E. M., Fluid Mechanics, AddisonWesley, (1959).

19. Houbolt, J. C., "A Recurrent Matrix Solution for the Dynamic Response of Elastic Aircraft," J. of Aeronautic Sciences, (Sept. 1950).

20. Swartzrauber, P. and Sweet, R., "Efficient Fortran Subprogram for the Solution of Elliptic Partial Differential Equations," NCAR-TN-IA-109, Nat'1, Center for Atmospheric Research, Boulder, Colorado, (July 1975).

21. Buzbee, B. L., "A Capacitance Matrix Technique," Sparse Matrix Computations Ed. J. R. Bunch \& D. J. Rose, Academic Press, (1976).

22. Madsen, N. K., Lawrence Livermore Laboratory, Private Communication, (1976-1977).

23. Posehn, M. R., Lawrence Livermore Laboratory, Private Communication, (1976-1977).

24. Kraus, H., Thin Elastic Shells, Wiley \& Sons, Inc., New York, (1967).

25. Cummings, G. and Feng, W. "Modeling Considerations in the Vibration Analysis of Reactor Internals, "Lawrence Livermore Laboratory Report UCRL-52023, (Feb. 1976). 
APPENDIX A - DERIVATION OF THE GOVERNING EQUATIONS

In this appendix we will derive the equations of motion for a circular cylindrical shell using Sanders' approximation of thin shell theory. We derive the governing equations for the fluid using cylindrical coordinates and assuming the fluid is inviscid and incompressible. An analytical solution of the fluid-shell problem will be described to explain the concept of "effective mass."

To reduce the dimensionality of the shell theory equations the dependent variables are expanded in Fourier series making the resulting set of equations applicable to an independent circumferential mode. The total solution is the sum of the Fourier series of the modal solutions. Likewise ary external forces must be expanded in a Fourier series and the appropinate Fourier coefficient of the force applied to the appropriate modal equations.

\section{SHELL EQUATIONS}

The derivation of the shell equations of motion will follow those of Kraus, Leissa, and Johnson and Greif $[A-1, A-2, A-3]$ based on a first approximation theory to thin shells developed by Sanders [A-4]. We found that Sanders' theory gave consistent results matching those found experimentally and analytically. The simpler Donnell method was also tried but we found it did not give consistant results near resonances. Therefore, since Sanders' method required anly a small increase in computer time to run a probiem compared to the Donnell method, it was used. Sanders formulates the shell theory equations using the principle uf virtual work with six "generalized displacements" and six "generalized forces." The generalized forces come from a force and moment balance on a shell element. Alternatively, Donnel1 [A-2] formulates the equations by dropping the transverse shear resultants $Q_{\xi}$ and $Q_{\theta}$ from the two tangential force balance equations and neglects the tangential displacements and their derivatives in the strain-displacement equations. 
To formulate the shell equations, we start by defining a thin shell as a three dimensional body bounded by two closely spaced curved surfaces, the distance between the surfaces being small in comparison with the other dimensions. The locus of points which lies midway between these surfaces is called the midsurface and the deformation of the shell will be completely determined by the displacements of this midsurface. We will be dealing with closed, circular cylindrical shells with uniform wall thickness. We will be assuming that the material of the shell is linearly elastic, isotropic, and homogeneous and that the effects of rotary inertia and shear deformation can be neglected.

We start by stating Love's postulates which are basic to all thin shell theories and which make use of Kirchhoff's hypothesis:

1. The shell is thin $(h / a<0.1)$

2. The deflections of the shell are small (linear theory holds)

3. The transverse normal stress can be neglected

4. Normals to the underformed midsurface remain straight and normal to the deformed midsurface and they, suffer no extension.

We will then sum the forces and moments which act on a shell element of thickness $h$ to form six equilibrium equations in terms of ten force and moment resultants. We make use of theprinciple of virtual work, using the equilibrium equations to formulate six generalized forces to go with the six generalized displacements. Integrating the virtual work equation gives the necessary strain-displacement relationship and Hooke's liw gives the necessary stress-strain relationships. By integrating the stresses across the thickness of the shell, employing Love's postulates, and using our known strain-displacement relationships we arrive at six force-displacement equations. Now reemploying the principle of virtual work we obtain three equations of equilibrium in terms of six force and moment resultants. Expanding the forces, moments, displacements, and strains in Fourier series 
we arrive at four equations of motion for each set of Fourier coefficients (1.e., each circumferential mode) in terms of the midsurface displacements $u, v, w$, and the axial bending moment $M_{\xi}$. The system of equations is eighth order, but by formulating the equations using four dependent variables the highest order der:vative is second order. By formulating the equations for each circumferiertial mode, we have reduced the number of dimensions to two, axial and time.

\section{Equations of Equilibrium}

Consider the equiiibrium of the shell element of thickness $h$, shown in Fig. 1, under the influence of the internal force and moment resultants shown. Also shown are the inertial and damping forces per unit surface area and an external surface load acting in the radial direction.

Our previous assumptions imply that the displacements, $u$ and $v$, and hence strain vary linearly through the shell thickness, We can therefore, with Hooke's law, integrate the resulting stresses over the shell thickness. The resultants of the integrals are the force and moment resultants per unit length.

Love's second postulate implies that the deflections are sufficiently small so that these force and moment resultants can be referred to the undeformed midsurface. Now if we apply Newton's law by summing the forces and moments which act upon the shell element we get the six equations of equilibrium.

$$
\begin{aligned}
& \frac{\partial N_{\xi}}{\partial \xi}+\frac{\partial N_{\theta \xi}}{\partial \theta}-a\left(\rho_{s} h \frac{\partial^{2} u}{\partial t^{2}}+\lambda \frac{\partial u}{\partial t}\right)=0 \\
& \frac{\partial N_{\theta}}{\partial \theta}+\frac{\partial N_{\xi \theta}}{\partial \xi}+Q_{\theta}-a\left(\rho_{s} h \frac{\partial^{2} v}{\partial t^{2}}+\lambda \frac{\partial v}{\partial t}\right)=0
\end{aligned}
$$




$$
\begin{aligned}
& -N_{\theta}+\frac{\partial Q_{\xi}}{\partial \xi}+\frac{\partial Q_{\theta}}{\partial \theta}-a\left(\rho_{s} h \frac{\partial^{2} w}{\partial t^{2}}+\lambda \frac{\partial w}{\partial t}-q\right)=0 \\
& \frac{\partial M_{\xi}}{\partial \xi}+\frac{\partial M_{\theta \xi}}{\partial \theta}-a Q_{\xi}=0 \\
& \frac{\partial M_{\theta}}{\partial \theta}+\frac{\partial M_{\xi \theta}}{\partial \xi}-a Q_{\theta}=0 \\
& N_{\xi \theta}-N_{\theta \xi}-\frac{M_{\theta \xi}}{a}=0
\end{aligned}
$$

In the above $N_{E}, N_{\xi \theta}, N_{\theta \xi}, N_{\theta}$ are the force resultants; $M_{\xi}, M_{E \theta}, M_{\theta \xi}$, $M_{\theta}$ are the moment resultants; $Q_{\xi}$ and $Q_{\theta}$ the transverse shear resultants; $u, v$, and $w$ the longitudinal, tangential, and radial midsurface displacements, $\rho_{s}$ the shell density, and $\lambda$, the viscous damping coefficient. The three independent variables are 1) a nondimenstonal length

$$
\xi \equiv x / a
$$

with $x$ the length coordinate and a the shell radius, 2) the circumferential angie $\theta$, and 3 ) the time $t$.

Principle of Virtual Work

We will now employ the principle of virtual work in terms of the six "generalized displacements" $u, v, w, \theta_{\xi}, \theta_{\theta}, \theta_{r}$ where $\theta_{\xi}, \theta_{\theta}$, and $\Theta_{r}$ are the rotations about the $\theta, \xi$, and $r$ directions respectively. The equilibrium equations $(A-1 a, \ldots f)$, neglecting body forces and surface loads and for a free shell, are used to obtain the "generalized forces" to go with the "generalized cisplacements". The principle of virtual work is thus written as, 


$$
\begin{aligned}
\int_{\xi} \int_{\theta} & {\left[\left(\frac{\partial N_{\xi}}{\partial \xi}+\frac{\partial N_{\theta \xi}}{\partial \theta}\right) \delta u+\left(\frac{\partial N_{\theta}}{\partial \theta \xi}+\frac{\partial N_{\xi \theta}}{\partial \theta}+Q_{\theta}\right) \delta v\right.} \\
& +\left(-N_{\theta}+\frac{\partial Q_{\xi}}{\partial \xi}+\frac{\partial Q_{\theta}}{\partial \theta}\right) \delta w+\left(\frac{\partial N_{\xi}}{\partial \xi}+\frac{\partial N_{\theta \xi}}{\partial \theta}-a Q_{\xi}\right) \delta \theta_{\xi} \\
& +\left(\frac{\partial M_{\theta}}{\partial \theta}+\frac{\partial M_{\xi \theta}}{\partial \xi}-a Q_{\theta}\right) \delta \theta_{\theta} \\
& \left.+a\left(N_{\xi \theta}-N_{\theta \xi}-\frac{H_{\theta \xi}}{\bar{a}}\right) \delta \theta_{r}\right] d \xi d \theta=0,
\end{aligned}
$$

$(A-2)$

with $\delta$ standing for "variation of", ide. $\delta u$ is the variation of $u$. Integrating $(A-2)$ by parts yields,

$$
\begin{aligned}
& \int_{\xi} \int_{\theta} {\left[N_{\xi} \delta\left(\frac{\partial u}{\partial \xi}\right)+N_{\xi \theta} \delta\left(\frac{\partial v}{\partial \xi}-a \theta_{r}\right)+N_{\theta \xi} \delta\left(\frac{\partial u}{\partial \theta}+a_{r}\right)\right.} \\
&+N_{\theta} \delta\left(\frac{\partial v}{\partial \theta}+w\right)+Q_{\xi} \delta\left(\frac{\partial w}{\partial \xi}+a \theta_{\xi}\right)+Q_{\theta} \delta\left(\frac{\partial w}{\partial \theta}-v+a_{\theta}\right) \\
&+M_{\xi} \delta\left(\frac{\partial \theta_{\xi}}{\partial \xi}\right)+M_{\xi \theta} \delta\left(\frac{\partial \theta_{\theta}}{\partial \xi}\right)+M_{\theta \xi} \delta\left(\frac{\partial \theta_{\xi}}{\partial \theta}+\theta_{r}\right) \\
&\left.+M_{\theta} \delta\left(\frac{\partial \theta_{\theta}}{\partial \theta}\right)\right] d \xi d \theta-\int_{C}\left\{\left[N_{\xi} \delta u+N_{\xi \theta} \delta v+Q_{\xi} \delta w\right.\right. \\
&+\left.M_{\xi} \delta \theta_{\xi}+M_{\xi \theta} \delta \theta_{\theta}\right] d \theta-\left[N_{\theta \xi} \delta u+N_{\theta} \delta v+Q_{\theta} \delta w\right. \\
&\left.\left.+M_{\theta \xi} \delta \theta_{\xi}+M_{\theta} \delta \theta_{\theta}\right] d \xi\right\}=0, \\
&-41-
\end{aligned}
$$

$(A-3)$ 
where the double integral extends over the midsurface of the shell enclosed by the curve $C$. The double integral represents the virtual change in strain energy within $C$ and the line iniegral represents the virtual work of the boundary forces. The quantities within the parentheses of $(f-3)$ can be regarded as the strains of the midsurface corresponding to the ten "generalized resultants" $N_{5}, \ldots, M_{\theta}$ thereby yielding the following straindisplacement relations.

$$
\begin{array}{ll}
\varepsilon_{\xi}=\frac{1}{a} \frac{\partial u}{\partial \xi}, \varepsilon_{\theta}=\frac{1}{a}\left(\frac{\partial v}{\partial \theta}+w\right), \gamma_{\xi}=\frac{1}{a} \frac{\partial v}{\partial \xi}-\theta_{r}, \gamma_{\theta}=\frac{1}{a} \frac{\partial u}{\partial \theta}+\theta_{r} & (A-4 a, b, c, d) \\
\kappa_{\xi}=\frac{1}{a} \frac{\partial \theta_{\xi}}{\partial \xi}, \kappa_{\theta}=\frac{1}{a} \frac{\partial \theta_{\theta}}{\partial \theta}, \kappa_{\xi \theta}=\frac{1}{a} \frac{\partial \theta_{\theta}}{\partial \xi}, k_{\theta \xi}=\frac{1}{a} \frac{\partial \theta_{\xi}}{\partial \theta}+\frac{\theta_{r}}{a} & (A-4 e, f, g, h) \\
\gamma_{\xi r}=\frac{1}{a} \frac{\partial w}{\partial \xi}+\theta_{\xi}, \gamma_{\theta r}=\frac{1}{a} \frac{\partial w}{\partial \theta}-\frac{v}{a}+\theta_{\theta}, & (A-4 i, j)
\end{array}
$$

where $E$ are the midsurface strains, $Y$ the shear strains, and $x$ the changes in midsurface curvature. Using Kirchhoff's hypothesis requires that $\gamma_{\xi r}=\gamma_{\theta r}=0$ giving

$$
\begin{aligned}
& \theta_{\xi}=-\frac{1}{a} \frac{\partial w}{\partial \xi}, \theta_{\theta}=\frac{v}{a}-\frac{1}{a} \frac{\partial w}{\partial \theta} \\
& \kappa_{\xi}=-; \frac{\partial^{2} w}{\partial \xi^{2}}, \kappa_{\theta}=-\frac{1}{a^{2}}\left(\frac{\partial^{2} w}{\partial \theta^{2}}-\frac{\partial v}{\partial \theta}\right) .
\end{aligned}
$$

He can put $\theta_{r}$ in terms of $u$ and $v$ by taking the norma $i$ component of the surface curl of the total displacement vector giving

$$
\theta_{r}=\frac{1}{2 a}\left(\frac{\partial v}{\partial \zeta}-\frac{\partial u}{\partial \theta}\right)
$$

From $(A-4, A-5)$ it can be shown that 


$$
\gamma_{\xi}=r_{\theta}, \kappa_{\xi \theta}-\kappa_{\theta \xi}=\frac{1}{2 a}\left(Y_{\xi}+\gamma_{\theta}\right),
$$

and if we define

$$
\begin{aligned}
& \varepsilon_{\xi \theta} \equiv \gamma_{\xi}+\gamma_{\theta}, \tau \equiv \kappa_{\xi \theta}+\kappa_{\theta \xi} \\
& \bar{N}_{\xi \theta} \equiv \frac{1}{2}\left(N_{\xi \theta}+N_{\theta \xi}\right)+\frac{1}{4 a}\left(M_{\xi \theta}-M_{\theta \xi}\right) \\
& \bar{M}_{\xi \theta} \equiv \frac{1}{2}\left(M_{\xi \theta}+M_{\theta \xi}\right),
\end{aligned}
$$

then using (A-5c through $A-6 f$ ) we can rewrite (A-3) as

$$
\begin{aligned}
\int_{\xi} \int_{\theta} & \left(N_{\xi} \delta \varepsilon_{\xi}+\bar{N}_{\xi \theta} \delta \varepsilon_{\xi \theta}+N_{\theta} \delta \varepsilon_{\theta}+M_{\xi} \delta \kappa_{\xi}+\bar{M}_{\xi \theta} \delta T+M_{\theta} \delta \kappa_{\theta}\right) \operatorname{ad} \xi d \theta \\
& -\int\left[\left(N_{\xi} \delta u+N_{\xi \theta} \delta v+Q_{\xi} \delta W+M_{\xi} \delta \theta_{\xi}+M_{\xi \theta} \delta \theta_{\theta}\right) d \theta\right. \\
& \left.-\left(N_{\theta \xi} \delta u+N_{\theta} \delta v+Q_{\theta} \delta w+M_{\theta \xi} \delta \theta_{\xi}+M_{\theta} \delta \theta_{\theta}\right) d \xi\right]=0
\end{aligned}
$$

From the double integral in (A-7), which represents the virtual change in strain energy, the generalized strains $\varepsilon_{\xi \theta}$ and $T$ corresponding to the resultants $\bar{N}_{\xi \theta}$ and $\bar{M}_{E \theta}$ are obtained.

$$
\varepsilon_{\xi \theta}=\frac{1}{a}\left[\frac{\partial u}{\partial \theta}+\frac{\partial v}{\partial \xi}\right], T=-\frac{1}{a^{2}}\left[2 \frac{\partial^{2} w}{\partial \theta \partial \xi}-\frac{3}{2} \frac{\partial v}{\partial \xi}+\frac{1}{2} \frac{\partial u}{\partial \theta}\right]
$$

We have thus reduced the original set of ten force and momentic resuitants to six and have arrived at six consistant strain-displacement 'elationships, equations ( $A-4 a, b ; A-5 c, d, A-8 a, b)$. The unique contribution of Sanders' theory is the expression for the midsurface twist, $T$. His formulation has ihe advantage that it results in zero strain for whole body rotation which is not the case for same of the other shell theories, e.g. Oonneli's approximation which neglects the tangential displacements and their 
derivatives in equations (A-5d and $A-8 b$ ). At the same time the six strain relationship's meet the requirements of Love's postulates and in particular Kirchhaff's hypothesis that the strains vary linearly with the shell thickness.

Force and Moment Resultants

Since the total strain, e, at an arbitrary point in the shell varies linearly with $\mathbf{r}$ (see $A-11 a, b, c$, ) we can integrate the resulting stresses over the shell thickness if we can define the relationship between stress and strain. Such a relationship is provided by Hooke's law which in three dimensional form is,

$$
\begin{aligned}
e_{\xi}= & \frac{1}{E}\left[\sigma_{\xi}-v\left(\sigma_{\theta}+\sigma_{r}\right)\right], e_{\theta}=\frac{1}{E}\left[\sigma_{\theta}-v\left(\sigma_{r}+\sigma_{\xi}\right)\right], \\
e_{r}= & \frac{1}{E}\left[\sigma_{r}-v\left(\sigma_{\xi}+\sigma_{\theta}\right)\right], \gamma_{\xi \theta}=\frac{2(1+v)}{E} \sigma_{\xi \theta}, \gamma_{\xi r}=\frac{2(1+v)}{E} \sigma_{\xi r}, \\
& \gamma_{\theta r}=\frac{2(1+v)}{E} \sigma_{\theta r} .
\end{aligned}
$$

From Kirchhoff's hypothesis we have $e_{r}=\gamma_{\xi r}=\gamma_{\theta r}=0$ giving us

$$
\begin{aligned}
& \sigma_{i}=\frac{E}{1-v^{2}}\left(e_{\xi}+v e_{\theta}\right), \sigma_{\theta}=\frac{E}{1-v^{2}}\left(e_{\theta}+v e_{\xi}\right), \\
& \sigma_{\xi \theta}=\frac{E}{2(1+v)} \gamma_{\xi \theta}
\end{aligned}
$$

If we now integrate the stresses over the faces of the shell element of Fig. 1 and neglect the $r / a$ term in comparison with unity we obtain expressions for the six force and mament resultants. 


$$
\begin{aligned}
& N_{\xi}=\int_{-h / 2}^{h / 2} \sigma_{\xi} d r, \quad N_{\theta}=\int_{-h / 2}^{h / 2} \sigma_{\theta} d r, \quad \bar{N}_{\xi \theta}=\int_{-h / 2}^{h / 2} \sigma_{\xi \theta} d r \quad(A-10 a, b, c) \\
& M_{\xi}=\int_{-h / 2}^{h / 2} r \sigma_{\xi} d r, \quad M_{\theta}=\int_{-h / 2}^{h / 2} r c_{\theta} d r, \quad \bar{N}_{\xi e}=\int_{-h / 2}^{h / 2} r \sigma_{z, j} d r \quad(A-30 d, e, f)
\end{aligned}
$$

Now from Kirchhoff's hypothesis we have that

$$
e_{\xi}=\varepsilon_{\xi}+r \kappa_{\xi}, e_{\theta}=\varepsilon_{\theta}+r \kappa_{\theta}, \gamma_{\xi \theta}=\varepsilon_{\xi \theta}+r T .
$$

Making the necessary substitutions from $(A-9, A-10)$ into $(A-11)$ and performing the integration gives a relationship between the six force and moment resultants and the six terms representing changes in midsurface strain, curvature, and twist.

$$
\begin{aligned}
& N_{\xi}=K\left(\varepsilon_{\xi}+v \varepsilon_{\theta}\right), N_{\theta}=K\left(\varepsilon_{\theta}+v \varepsilon_{\xi}\right), \bar{N}_{\xi \theta}=K(1-\nu) \varepsilon_{\xi \theta} \quad(A-12 a, b, c) \\
& M_{\xi}=D\left(\kappa_{\xi}+v \kappa_{\theta}\right), M_{\theta}=D\left(\kappa_{\theta}+v \kappa_{\xi}\right), \bar{M}_{\xi \theta}=\frac{D}{2}(1-\nu) T \quad(A-12 d, e, f) \\
& \text { where } \quad D \equiv \frac{h^{3} E}{\frac{12\left(T-v^{2}\right)}{K}} \quad K \equiv \frac{h E}{\left(T-v^{2}\right)}
\end{aligned}
$$

Equations of Motions

We are now in a positon to arrive at the equations of motion of a circular cylindrical shell in terms of Sanders' six force and moment resultants as opposed to the eight resultants in the origilal equilibrium equations ( $A-l a, b, c, d, e, f)$. We do this by again applying the principle of virtual work.

Beginning with the virtual change in strain energy due to the internal force and moment resultants as given by ths area integral in (A-7), reintroducing the transverse shear resultants $Q_{E}$ and $Q_{\theta}$, and integrating by parts gives 


$$
\begin{aligned}
\int_{\xi} \int_{\theta}\left(N_{\xi} \delta \varepsilon_{\xi}+\bar{N}_{\xi \theta} \delta \varepsilon_{\xi \theta}+N_{\theta} \delta \varepsilon_{\theta}+M_{\xi} \delta x_{\xi}+\bar{M}_{\xi \theta} \delta T+M_{\theta} \delta \kappa_{\theta}+Q_{\xi} \delta \gamma_{\xi r}\right. \\
\left.\quad+Q_{\theta} \delta \gamma_{\theta r}\right)_{a} d_{\xi} d_{\theta}=\int_{C}\left[\left\{N_{\xi} \delta u+\bar{N}_{\xi \theta} \delta v+Q_{\xi} \delta w\right.\right. \\
\left.+M_{\xi} \delta \theta_{\xi}+\bar{M}_{\xi \theta} \delta\left[\theta_{\theta}+\frac{v}{2 a}\right]\right\} d \theta-\left\{N_{\theta} \delta v+\bar{N}_{\xi \theta} \delta u\right. \\
\left.\left.+Q_{\theta} \delta w+M_{\theta} \delta \theta_{\theta}+\bar{M}_{\xi \theta} \delta\left[\theta_{\xi}+\frac{u}{2 a}\right]\right\} d \xi\right] \\
\quad-\int_{\xi} \int_{\theta}\left(F_{1} \delta u+F_{2} \delta v+F_{3} \delta w+F_{4} \delta \theta_{\xi}+F_{5} \delta \theta_{\theta}\right) d \xi d \theta
\end{aligned}
$$

where

$$
\begin{aligned}
& F_{1}=\frac{\partial N_{\xi}}{\partial \xi}+\frac{\partial N_{\xi \theta}}{\partial \theta}-\frac{1}{2 a} \frac{\partial \bar{M}_{\xi \theta}}{\partial \theta} \\
& F_{2}=\frac{\partial N_{\theta}}{\partial \theta}+\frac{\partial \bar{N}_{E \theta}}{\partial \xi}+\frac{1}{2 a} \frac{\partial \bar{M}_{\xi \theta}}{\partial \xi}+Q_{\theta} \\
& F_{3}=-N_{\theta}+\frac{\partial Q_{\xi}}{\partial \xi}+\frac{\partial Q_{\theta}}{\partial \theta} \\
& F_{4}=\frac{\partial M_{\xi}}{\partial \xi}+\frac{\partial \bar{M}_{\xi \theta}}{\partial \theta}-a Q_{\xi} \\
& F_{5}=\frac{\partial M_{\theta}}{\partial \theta}+\frac{\partial \bar{M}_{E \theta}}{\partial \xi}-a Q_{\theta}
\end{aligned}
$$

For the shell to be in equilibrium, the principle of virtual work requires that the lefthand side of (A-13a) must be equal to the line integral on the right-hand side, in which case the area integral on the right-hand side must vanish. Because the virtual displacements $\delta u, \ldots \ldots, \delta \theta_{\theta}$ are independent and arbitrary, each term of the area integral must vanish independently. Thus, setting

$$
F_{1}=F_{2}=F_{3}=F_{4}=F_{5}=0
$$


in (A-13) gives the modified equations of equilibrium for the internal forces resulting from Sanders' theory. Adding back to the equilibrium equations the appropriate inertial, damping, and surface load terms gives the corresponding equations of motion.

One additional step reduces the number of equations of motion from five to three. This is done by solving $(A-13 \mathrm{e}, \mathrm{f})$ for $Q_{\xi}$ and $Q_{\theta}$ and substituting these results into $(A-13 b, c, d)$ giving

$$
\begin{gathered}
\frac{\partial N_{\xi}}{\partial \xi}+\frac{\partial N_{\xi \theta}}{\partial \theta}-\frac{1}{2 a} \frac{\partial \bar{M}_{E \theta}}{\partial \theta}-\underline{E} \frac{\partial^{2} u}{\partial \tau^{2}}-\lambda \sqrt{\frac{E}{\rho_{s}}} \frac{\partial u}{\partial \tau}=0 \\
\frac{\partial N_{\theta}}{\partial \theta}=\frac{\partial \bar{N}_{\xi \theta}}{\partial \xi}+\frac{3}{2 a} \frac{\partial \bar{M}_{\xi \theta}}{\partial \xi}+\frac{1}{a} \frac{\partial M_{\theta}}{\partial \theta}-\frac{E h}{a} \frac{\partial^{2} v}{\partial \tau^{2}}-\lambda \sqrt{\frac{E}{\rho_{s}}} \frac{\partial v}{\partial \tau}=0 \\
-N_{\theta}+\frac{1}{a}\left[\frac{\partial^{2} M_{\xi}}{\partial \xi^{2}}+2 \frac{\partial^{2} \bar{M}_{\xi \theta}}{\partial \xi \partial \theta}+\frac{\partial^{2} M_{\theta}}{\partial \theta^{2}}\right]-\frac{E h}{a} \frac{\partial^{2} w}{\partial \tau^{2}} \\
-\lambda \sqrt{\frac{E}{\rho_{s}}} \frac{\partial W}{\partial \tau}+a q=0,
\end{gathered}
$$

where we have introduced the dimensionless time variable, $\tau$.

$$
\tau \equiv \sqrt{\frac{E}{\rho_{s}}} \frac{t}{a}
$$

Boundary Conditions

The natural boundary conditions associated with Sanders' equations of equilibrium are obtained by setting the virtual work of the forces acting on the boundaries of the shell equal to zero. Thus from (A-13a) we get at the ends of the cylinder $(\xi=0, s / a)$,

* Number refers to equations in main body of report 


$$
\begin{array}{ll}
\hat{N}_{E} & \text { or } u=0 \\
\hat{N}_{\xi \theta} \equiv\left[\bar{N}_{E \theta}+\frac{3}{2 a} \bar{M}_{E \theta}\right] & \text { cr } y=0 \\
\hat{Q}_{E} \equiv\left[\frac{\partial H_{\xi}}{\partial E}+2 \frac{\partial N_{E \theta}}{\partial \theta}\right] & \text { or } \omega=0 \\
M_{E} & \text { or } \theta_{\xi}=0
\end{array}
$$

where $\hat{N}_{\xi \theta}$ and $\hat{Q}_{\xi}$ can be thought of as effective membrane and transverse shear resultants per unit length. Any combination of four conditions must be prescribed at each end of the cylinder.

\section{Fourier Series Expansion}

Our ultimate goal is to arrive at a set of four dimensioniess, coupled, partial differential equations in terms of the four dependent variables, $u, v, w, M_{F}$ and the two independent yariables $\xi$ and $\tau, a$ dimensiontess space and time. In order to do this we first expand all the force and moment resultants, displacements, and strains into Fourier series in the circumferential variable $\theta$ to eliminate the $\theta$ dimension. We then make use of a reference stress level, $\sigma_{0}$, to make all the Fourier coefficients $=$ dimensionless.

$$
\begin{array}{lll}
N_{\xi}=\sigma_{0} h \sum_{n=0}^{\infty} N_{\xi n} \cos n \theta & N_{\theta}=\sigma_{0} h \sum_{n=0}^{\infty} N_{\theta n} \cos n \theta \quad & (A-15 a, b) \\
\bar{H}_{\xi \theta}=\sigma_{0} h \sum_{n=1}^{\infty} N_{\xi \theta n} \sin n \theta & & (A-15 c) \\
M_{\xi}=\frac{\sigma_{0} h^{9}}{a} \sum_{n=0}^{\infty} M_{\xi n} \cos n \theta & N_{\theta}=\frac{\sigma_{0} h^{3}}{a} \sum_{n=0}^{\infty} M_{\theta n} \cos n \theta \quad \text { (3d,A-15d) }
\end{array}
$$




$$
\begin{aligned}
& \overline{\mathbf{H}}_{\xi \theta}=\frac{\sigma_{0} h^{3}}{a} \sum_{n=1}^{\infty} M_{\xi \theta n} \sin n \theta \\
& u=\frac{\partial \sigma_{0}}{E} \sum_{n=0}^{\infty} u_{n} \cos n \theta \quad v=\frac{\partial \sigma_{0}}{E} \sum_{n=1}^{\infty} v_{n} \sin n \theta \\
& w=\frac{a \sigma_{0}}{E} \sum_{n=0}^{\infty} w_{n} \cos n \theta \\
& \theta_{\xi}=\frac{\sigma}{E} \sum_{n=0}^{\infty} \theta_{\xi n} \cos n \theta \\
& \varepsilon_{\xi}=\frac{\sigma_{0}}{E} \sum_{n=0}^{\infty} \varepsilon_{\xi n} \cos n \theta \\
& \varepsilon_{\theta}=\frac{\sigma_{0}}{E} \sum_{n=0}^{\infty} \varepsilon_{\theta n} \cos n \theta \\
& (A-16 b, c) \\
& \varepsilon_{\xi \theta}=\frac{\sigma_{0}}{E} \sum_{n=1}^{\infty} c_{\xi \in n} \sin n \theta \\
& \kappa_{\xi}=\frac{\sigma_{0}}{\mathrm{a} E} \sum_{n=0}^{\infty} \kappa_{\xi n} \cos n \theta \\
& \kappa_{\theta}=\frac{\sigma}{a E} \sum_{n=0}^{\infty} \kappa_{\theta n} \cos n \theta \\
& (A-16 e, f) \\
& T=\frac{\sigma_{0}}{\mathrm{aE}} \sum_{n=1}^{\infty} T_{n} \sin n \theta \\
& \text { (A-16g) } \\
& q=\frac{\sigma_{D} h}{a} \sum_{n=0}^{\infty} q_{n} \cos n \theta
\end{aligned}
$$

If now $(3, A-15)$ are substituted into (1) we obtain the three equations of motion uncoupled in the Fourier index $n$. The equations of motion become

$$
\begin{gathered}
N_{\xi n}^{\prime}+n N_{\xi \theta n}-\frac{n h^{2}}{2 a^{2}} H_{\xi \theta n}-\ddot{u}_{n}-\dot{a}_{n}=0 \\
N_{\xi \theta n}-n N_{\theta n}-\frac{n h^{2}}{a^{2}} M_{\theta n}+\frac{3 h^{2}}{2 a^{2}} M_{\xi \theta n}^{\prime}-v_{n}-C v_{n}=0 \quad(A-17 b) \\
-N_{\theta n}+\frac{h^{2}}{a^{2}}\left(M_{\xi n}^{\prime \prime}-n^{2} M_{\theta n}+2 n M_{\xi \theta n}^{\prime}\right)-\ddot{H}_{n}-\dot{w}_{n}+q_{n} \\
-49- \\
\text { (A-17c) }
\end{gathered}
$$


where the variables are tics dimensionless Fourier coefficients. The derivatives with respect to $\xi$ and $\tau$ are denoted by primes and dots, respectively. $C$ is a dimensionless damping coefficient. The rotation, strain-displacement, and stress-strain relations for each $\boldsymbol{n}$ are obtained in a similar manner using $(A-4 a, b, ; A-5 a, c, d ; A-8 a, b, ; A-12 a, b, c, d, e, f)$ and $(3, A-15, A-16)$ as appropriate. Making the necessary substitutions we get

$$
\begin{aligned}
& \varepsilon_{\xi n}=u_{n}^{\prime}, \varepsilon_{\theta n}=n v_{n}+w_{n}, \varepsilon_{\xi \theta n}=1 / 2\left[v_{n}^{\prime}-n u_{n}\right](A-18 a, b, c) \\
& \kappa_{E n}=-w_{n}^{\prime \prime}, k_{\theta n}=n^{2} w_{n}+n v_{n}, \theta_{\xi n}=-w_{n}^{\prime} \quad(A-18 d, e, f) \\
& T_{n}=2 n w_{n}^{\prime}+\frac{3}{2} v_{n}^{\prime}+\frac{n}{2} u_{n} \\
& (A-18 g) \\
& N_{\xi n}=\frac{1}{1-v^{2}}\left(\varepsilon_{\xi n}+v \varepsilon_{\theta n}\right) \\
& (A-18 h) \\
& N_{\theta n}=\frac{1}{1-v^{2}}\left(\varepsilon_{\theta}+v \varepsilon_{\xi n}\right), N_{\xi \theta n}=\frac{1}{1+v} \varepsilon_{\xi \theta n} \\
& (A-18 i, j) \\
& H_{\xi n}=\frac{1}{12\left(1-v^{2}\right)}\left(\kappa_{\xi n}+v k_{\theta n}\right), \\
& \text { (A-18k) } \\
& M_{\theta n}=\frac{1}{12\left(1-v^{2}\right)}\left(\kappa_{\theta n}+v k_{E n}\right), M_{\xi \theta n}=\frac{1}{24(1+v)} T_{n} \\
& (A-181, m)
\end{aligned}
$$

$-50-$ 
Substitution of equations $(A-18, a, \ldots, m)$ into equations $(A-17 a, b, C)$ will lead to three coupled partial differential equations ...th $u_{n}, v_{n}$, and $w_{n}$ as the dependent variables. The highest order derivative will be a term containing the fourth derivative of $w_{n}$. However, it will be convenient to limit the highest order space derivative to the second order. Consequently, $u_{n}, v_{n}, w_{n}$, and $M_{\xi n}$ will be used as dependent variables and four coupled partial differential equations will be derived. The definition of $M_{\xi n}$ (equation A-18K) in terms of $\kappa_{E n}$ (equation A-18d) and $k_{\theta n}$ (eruation A-T6e) provides the fourth equation. The desired equations of motion for a circular cylindrical shell for one set of Fourier components is thus

$$
\begin{aligned}
& \alpha_{1} u_{n}^{\prime \prime}+\alpha_{2} u_{n}+\alpha_{3} v_{n}^{\prime}+\alpha_{4} w_{n}^{\prime}=u_{n}+c \dot{u}_{n} \\
& \alpha_{5} u_{n}^{\prime}+\alpha_{6} v_{n}^{\prime \prime}+\alpha_{7} v_{n}+\alpha_{8} w_{n}^{\prime \prime}+\alpha_{9} w_{n}+\alpha_{10} \mu_{E n} \\
& =v_{n}+\dot{c i}_{n} \\
& \alpha_{11} u_{n}^{\prime}+\alpha_{12} v_{n}^{\prime \prime}+\alpha_{13} v_{n}+\alpha_{14} w_{n}^{\prime \prime}+\alpha_{15} w_{n}+\alpha_{16} M_{5 n}^{\prime \prime} \\
& +\alpha_{17} M_{\xi n}=\ddot{w}_{n}+\dot{c}_{n}-q_{n} \\
& \alpha_{18} v_{n}+\alpha_{19} w_{n}^{\prime \prime}+\alpha_{20} w_{n}+\alpha_{21} M_{E n}=0 .
\end{aligned}
$$

The coefficients for these equations are shown in Table A-1.

The boundary conditions (4) must also be described in terms of Fourier components. To do this we first expand the effective membrane and transvarse shear resultants into fourier series with dimensionless coefficients. 
TABLE AN

Formulas for Coefficients of Shell

Equations of Motion, Ens aa,.., ad

$$
\begin{aligned}
& \alpha_{1}=\frac{1}{1-v^{2}} \quad \alpha_{11}=-\alpha_{4} \\
& \alpha_{2}=-\frac{n^{2}}{2(1+v)}\left[1+\frac{n^{2}}{48 a^{2}}\right] \quad \alpha_{12}=\alpha_{8} \\
& \alpha_{3}=\frac{n}{2}\left[\frac{1}{1-v}-\frac{h^{2}}{16 a^{2}(1+v)}\right] \quad \alpha_{13}=\alpha_{9} \\
& \alpha_{4}=\frac{v}{1-v^{2}}-\frac{n^{2} h^{2}}{24 a^{2}(1+v)} \quad \alpha_{14}=\frac{n^{2} h^{2}}{6 a^{2}(1+v)} \\
& \alpha_{5}=-\alpha_{3} \quad \alpha_{15}=-\left[\frac{1}{1-v^{2}}+\frac{n^{4} h^{2}}{12 a^{2}}\right] \\
& \alpha_{6}=\frac{1}{2(1+v)}\left[1+\frac{3 h^{2}}{16 a^{2}}\right] \quad \alpha_{16}=\frac{h^{2}}{a^{2}} \\
& \alpha_{1}=-n^{2}\left[\frac{1}{1-\nu^{2}}+\frac{h^{2}}{12 a^{2}} j \quad \alpha_{17}=n \alpha_{10}\right. \\
& \alpha_{8}=\frac{n h^{2}}{8 a^{2}(1+v)} \\
& \alpha_{18}=\frac{n v}{12\left(7-v^{2}\right)} \\
& \alpha_{9}=-\frac{n}{1-v^{2}}-\frac{n^{3} h^{2}}{12 a^{2}} \quad \alpha_{19}=-\frac{1}{12\left(1-v^{2}\right)} \\
& a_{10}=-\frac{v n h^{2}}{a^{2}} \\
& \alpha_{21}=-1 \\
& \alpha_{20}=n \alpha_{18} \\
& -52-
\end{aligned}
$$




$$
\begin{aligned}
& \hat{n}_{\xi \theta}=\sigma_{0} h \sum_{n=1}^{\infty} \hat{N}_{\xi \theta n} \sin n \theta \\
& \hat{q}_{\xi}=\sigma_{0} h \sum_{n=0}^{\infty} \hat{Q}_{\xi n} \cos n \theta
\end{aligned}
$$

Now making use of the relationships describing $\hat{N}_{\xi \Theta}$ and $\hat{O}_{\xi}$ (4) for arbitrary n we have

$$
\begin{aligned}
& \hat{N}_{\xi \theta n}=\bar{N}_{\xi \theta n}+\frac{3 h^{2}}{2 a^{2}} \bar{M}_{\xi \theta n} \\
& \hat{Q}_{E n}=\frac{h^{2}}{a^{2}}\left[M_{\xi n}^{\prime}+2 n \bar{M}_{\xi \theta n}\right]
\end{aligned}
$$

The right hand side of these equations can be put in terms of displacements and it follows from (A-18) that

$$
\begin{aligned}
& N_{\xi n}=B_{1} U_{n}^{\prime}+B_{2} V_{n}+B_{3} W_{n} \\
& \hat{N}_{E \theta n}=B_{4} U_{n}+B_{5} V_{n}^{\prime}+B_{6} W_{n}^{\prime} \\
& \hat{Q}_{\xi n}=B_{7} U_{n}+B_{8} V_{n}^{\prime}+B_{9} W_{n}^{\prime}+B_{10} W_{\xi n}^{\prime}
\end{aligned}
$$

where the coefficients, $B$, are shown in Table A-2. The boundary conditions may now be rewritten, for the nth fourier component, as prescribing the following dimensionless quantities at the ends of the cylinder, $\xi=0, s / a$.

$$
\begin{array}{ll}
N_{\xi n} \text { or } & U_{n}=0 \\
\hat{N}_{\xi \in n} \text { or } & v_{n}=0
\end{array}
$$


Table A-2

Formulas for the Coefficients of the End Condition Equations, Equations A-21a,b,c

$$
\begin{array}{ll}
\beta_{1}=\frac{1}{1-v^{2}} & \beta_{6}=\frac{n h^{2}}{8 a^{2}(1+v)} \\
\beta_{2}=\frac{u n}{1-v^{2}} & B_{7}=\frac{n}{8} \beta_{6} \\
\beta_{3}=\frac{v}{1-v^{2}} & \beta_{8}=\beta_{6} \\
\beta_{4}=-\frac{n}{2(7+v)}\left[-1+\frac{h^{2}}{16 a^{2}}\right] & \beta_{9}=\frac{4}{3} n \beta_{6} \\
\beta_{5}=\frac{1}{2(7+v)}\left[1+\frac{3 h^{2}}{16 a^{2}}\right] & B_{10}=\frac{h^{2}}{a^{2}}
\end{array}
$$

$(A-22 C)$

$$
\begin{array}{ll}
\hat{q}_{\xi n} \text { or } & w_{n}=0 \\
M_{\xi n} \text { or } & \theta_{\xi n}=0
\end{array}
$$

FLUID EQUATIONS

The assumption will be made that the fluid is inviscid, incompressidle, and has no gross motion. With these assumptions the fluid can be represented by one dependent variable, the dynamic pressure p. We will

$-54-$ 
expand $p$ into a Fourier series and in turn obtain a differential equation for each Fourier component of $p$ in terms of the independent variables $\xi, r, \tau$, where $r$ is a dimensionless radial coordinate, $r=r / a$. Each such Fourier component of the dynamic pressure, when evaluated at the cylinder wall, will act as an additional shell loading to be included with the equations of motion for the Fourier components of the shell variables. This implies that the pressure belonging to a given circumferential mode does not excite other than its matching structural mode, an assumption usually made in modat analysis.

We start with the equation of motion for inviscid, compressible fluids (Euler's equation) and the continuity equation. [A-5] .

$$
\begin{aligned}
& \rho_{f}\left(\frac{\partial}{\partial t}+v \cdot \nabla\right) v+\nabla p_{t}=0 \\
& \frac{\partial \rho_{f}}{\partial t}+\operatorname{div}\left(\rho_{f} V\right)=0
\end{aligned}
$$

Here we are taking $\rho_{f}$ as the fluid density, $V$ as the fluid velocity, and $p_{t}$ as the total fluid pressure. For small osillations we assume the conyective terms $(V \cdot \nabla) V$ can be neglected. Now if we assume the density, velocity, and pressure are made up of main plus perturbed components,

$$
\begin{aligned}
& \rho_{f}=\rho_{0}+\rho_{s}^{\prime} V=V_{0}+V^{\prime}, p_{t}=p_{0}+p \text { and assuming } \\
& p_{t}>p, \rho_{0} \gg \rho^{\prime} \text { we have } \\
& \frac{\partial V^{\prime}}{\partial t}+\frac{1}{p_{f}} \text { grad } p=0 \\
& \frac{\partial \rho^{\prime}}{\partial t}+\rho_{f} \text { div } V^{\prime}=0 .
\end{aligned}
$$


These equations will hold if $V^{\prime}<<$, the accl!stis velocity oi lits iluic. Typically, for instance in a pressurized water reactor, we are interested in $V^{\prime}<<1000 \mathrm{in} / \mathrm{sec}$ and in general $c>45,000 \mathrm{in} / \mathrm{sec}$.

Assuming adiabatic flow, the wave motion in the fluid can be represented by

$$
p / \rho_{f}^{\prime}=c^{2} \text {. }
$$

If we solve $(A-25)$ for $p$ and substitute into $(A-24 b)$, differentiate the result with respect to time and substitute in the equivalent of $\partial \mathrm{V}^{\mathrm{r}} / \partial \mathrm{t}$ from (A-24a) we obtain the wave equation in terms of the dynamic pressure, $p$.

$$
\nabla^{2} p=\frac{1}{c^{2}} \quad \frac{\partial^{2} p}{\partial t^{2}}
$$

Assuming the fluid incompressible requires that $c+\infty$ and so the time derivative drops out. If we now represent (5) in cylindrical coordinates we obtain

$$
\frac{\partial^{2} p}{\partial r^{2}}+\frac{1}{r} \frac{\partial p}{\partial r}+\frac{1}{r^{2}} \frac{\partial^{2} p}{\partial \theta^{2}}+\frac{\partial^{2} p}{\partial \xi^{2}}=0
$$

We will now expand $p$ in a Fourier series and make the Fourier coefficients dimensionless giving

$$
p=\frac{\sigma_{0} h}{a} \sum_{n=0}^{\infty} p_{n} \cos n \theta
$$

If we substitute (A-27) into (A-26) we get our desired dimensionless equation in terms of the nth Fourier component of the dynamic pressure.

$$
\frac{\partial^{2} p_{n}}{\partial r^{2}}+\frac{1}{r} \frac{\partial p_{n}}{\partial r}-\frac{n^{2}}{r^{2}} p_{n}+\frac{\partial^{2} p_{n}}{\partial \xi^{2}}=0
$$




\section{Boundary Conditions}

Equation (6) is a form of the Helmholtz equation and requires boundary conditions to be specified over the top and bottom and on the inner and outer surfaces of the fluid region. Over the top or bottom either Dirichlet $(p=0)$ or Neumann $(\partial p / \partial \xi=0)$ boundary conditions can be employed depending on whether the top and bottom are open or closed.

$$
\begin{array}{lll}
\left.p_{n}\right|_{\xi=0}=0 & \text { or } & \left.\frac{\partial p_{n}}{\partial \xi}\right|_{\xi=0}=0 \\
\left.P_{n}\right|_{\xi=s / a}=0 & \text { or } & \left.\frac{\partial p_{n}}{\partial \xi}\right|_{\xi=s / a}=0
\end{array}
$$

We will note from Fig. I that we have two fluid regions to consider, the fluid inside the cylinder and the fluid outside. For the inmer fluid we neglect the fluid inside small radius $r_{0}$ so as to eliminate the singularity at $r=0$. One way to determine the location of $r_{0}$ is by calculating the "effective mass" $[A-6]$ needed to account for the she 11 's response when filled with fluid and calculating what fluid thickness this represents. This fluid thickness is then subtracted from the shell radius to get $r_{0}$.

The outer radius of the inner fluid is the shell wall and the adhesion condition requires that the shell and fluid radial velocities match over this surface. If we define a velocity potential of the perturbed velocity as $V^{\prime}=$ grad $\phi$ then we have for the adhesion condition that

$$
\frac{\partial w}{\partial t}=\left.\frac{\partial \phi}{\partial r}\right|_{r=a}
$$


and from $(A-24 a)$ that

$$
p=-\rho_{0} \frac{\partial \phi}{\partial t}
$$

Combining these and using the nondimensional variables gives

$$
\frac{\partial p_{n}}{\partial r}= \pm \frac{a}{h} \frac{\rho_{f}}{\rho_{s}} \ddot{w}_{n}
$$

as a representation of the adhesion boundary coldition.

For the inner fluid the radial boundary conditions are

$$
\left.\frac{\partial p_{n}}{\partial r}\right|_{r=0 / a}=0 \text { and }\left.\frac{\partial p_{n}}{\partial r}\right|_{r=1}=\frac{a}{h} \frac{\rho_{f}}{\rho_{s}} \ddot{H}_{n}
$$

For the outer fluid the radial boundary conditions are

$$
\left.\frac{\partial p_{n}}{\partial r}\right|_{h=b / a}=0 \text { and }\left.\frac{\partial p_{n}}{\partial r}\right|_{r=1}=-\frac{a}{h} \frac{\rho_{f}}{\rho_{s}} \ddot{w}_{n}
$$

where $b$ is the outside boundary of the outer fluid.

\section{ANALYTICAL SOLUTION}

To explore the region over which the incompressibility assumption is valid and to derive an expresston for the "effective mass" ve will examine an analytical solution to the flutd-shell problem. Ne will, in general, follow the solution techniques presented by Mnev and Pertsev [A-7] for an infinitely long (or simply-supported) circular cylindrical shell surrounded by a thin annular fluid region whose outer boundary is rigid. The previous assumptions of Love's postulates for the shell and an inviscid, stationery fluid will be retained. However, for present purposes, the fluid will be assumed compressible. 
We start with the three shell equations of motion described in terms of the shell midsurface displacements $u, v$, and $w$. We will assume no external load on the shell other than that due to the fluid's resistance to shell motion and no damping. He obtain

$$
\begin{aligned}
& L_{11} u+L_{12} v+L_{13^{w}}=\rho_{s} h \frac{\partial^{2} u}{\partial t} \\
& L_{21} u+L_{22} v+L_{23^{w}}=\rho_{s} h \frac{\partial^{2} v}{\partial t^{2}} \\
& L_{31} u+L_{32} v+L_{33^{w}}=\rho_{s} h \frac{\partial^{2} w}{\partial t^{2}}-p
\end{aligned}
$$

where $p$ is the fluid dynamic pressure and is negative if the pressure is external. The $L_{, j}$ are linear operators defined by the particular formulation of the shell equations used.

The fluid is described by the acoustic wave equation which for convenience has been cast in terms of the velocity potential, $\phi$. The equation and boundary conditions are

$$
\begin{gathered}
\nabla^{2} \phi=\frac{\partial^{2} \phi}{\partial r^{2}}+\frac{1}{r} \frac{\partial \phi}{\partial r}+\frac{1}{r^{2}} \frac{\partial^{2} \phi}{\partial \theta^{2}}+\frac{\partial^{2} \phi}{\partial x^{2}}=\frac{1}{c^{2}} \frac{\partial^{2} \phi}{\partial t^{2}} \\
\left.\frac{\partial \phi}{\partial r}\right|_{r=a}=\frac{\partial w}{\partial t},\left.\frac{\partial \phi}{\partial r}\right|_{r=b}=0
\end{gathered}
$$

Equation (A-33) will admit solutions of the form

$$
\begin{aligned}
& u=u_{0} \cos \frac{\pi m x}{s} \cos n \theta \sin \theta t \\
& v=v_{0} \sin \frac{\pi m x}{s} \sin n \theta \sin \Omega t \\
& w=w_{0} \sin \frac{\pi m x}{s} \cos n \theta \sin \Omega t
\end{aligned}
$$


where $u_{0}, v_{0}, w_{0}$ are constants, $m$ is the number of axial nodes less one, and $\Omega$ is the natural frequency of vibration. He can represent $\phi$ by

$$
\hat{\theta}=T(t) k(r) \sin \frac{\pi m x}{s} \cos n \theta
$$

where $T(t)$ and $R(r)$ are yet to be determined. The adhesion condition can be used to determine $T(t)$ giving

$$
T(t)=\frac{\Omega w_{0}}{\mathrm{dR} / \mathrm{dr} \mid \mathrm{r}=\mathrm{a}} \cos \Omega \mathrm{t}
$$

If we substitute $(A-35 d)$ into $(A+34)$ and make use of (A-36) we obtain a Bessel equation of order $n$.

$$
r^{2} \frac{\partial^{2} R}{\partial r^{2}}+r \frac{\partial R}{\partial r}-\left[\left(\frac{\pi m \zeta r}{5}\right)^{2}+n^{2}\right] R=0
$$

The solution is in the form of nth order Bessel functions

$$
R(r)=\left\{\begin{array}{l}
A_{1} I_{n}\left(\frac{\pi m \zeta r}{s}\right)+A_{2} K_{n}\left(\frac{\pi m \zeta r}{s}\right), \Omega<\frac{c \pi}{s} \\
B_{1} J_{n}\left(\frac{\pi m \bar{\zeta} r}{s}\right)+B_{2} Y_{n}\left(\frac{\pi m \bar{\zeta} r}{s}\right), \Omega>\frac{c \pi}{s}
\end{array}\right.
$$

where $J_{n}, Y_{n}$ are Bessel functions of the first and second $k i n d$ and $L_{n}, K_{n}$ are modified Bessel functions of the first and second kind. Here we define

$$
\zeta \equiv \sqrt{1-\left(\frac{\Omega s}{\pi c n}\right)^{2}} \text { and } \bar{\zeta}=\sqrt{-T} \zeta
$$

Using the boundary condition at the outer fluid boundary we find that

$$
\phi(x, r, \theta)=w_{0} \Omega \cos \Omega t \sin \frac{\pi m x}{s} \cos n \theta \frac{R / r=b}{\partial R / \partial r]_{r=a}}
$$


and If we differentiate with respect to time and substitute into (A-31) we obtain

$$
\left.p\right|_{r=a}=-\rho_{f} \frac{\partial \phi}{\partial t}=\rho_{f} w_{0} \Omega^{2} \sin \Omega t \sin \frac{\pi m x}{s} \cos n \theta \frac{\left.R\right|_{r=b}}{\partial R /\left.\partial r\right|_{r=a}}
$$

If we substitute this expression for the fluid dynamic pressure into the third shell equation of motion $(A-33 C)$ we arrive at

$$
L_{31} u+L_{32} v+L_{33} w=\left(\rho_{s} h+\rho_{f} \Phi\right) \frac{\partial^{2} w}{\partial t^{2}}
$$

where we define

$$
\phi(n, m, a, b, \zeta, s)=-\frac{R(b)}{\partial R /\left.\partial r\right|_{r=a}}
$$

The term $\rho_{f} \Phi$ characterizes the inertial counteraction of the medium to the vibration of the shell within the limits of the given natural frequency for that mode and can be termed the connected mass of the fluid. He will note that $\Phi$ depends on the circumferential mode number $n$, the axial mode number $m$, shell length $s$, shell radius $a$, outer flutd radius $b$, and the compress $i-$ bility factor $\zeta$. If the shell is excited primarily in one mode then its motion could be properly calculated by replacing the normal mass, $\rho_{s} h$, by an "effective mass" Meff, equal to the sum of the normal shell mass and the connected mass of the fluid for the mode.

$$
M_{\mathrm{eff}}=\rho_{s} h+\rho_{f} Q
$$


When using thin shell theory it has been shown [A-1] that, in vacuo three natural frequencies are excited for each $(n, m)$ mode, the lowest of which causes predomirately radial motion. Mnev and Pertser [A-7] and Bleich and Baron [A-8] have shown that when the shell is immersed in a dense fluid only this lowesi frequency is of consequence. With these simplifications the natural frequency of each mode in a fluid is related to the shell natural frequencies in vacuo by

$$
\Omega=\frac{\omega}{\sqrt{1+\frac{\rho_{f} \phi}{\rho_{s} h}}}
$$

where $w$ is the lowest natural frequency of the $(n \cdot 1$ mode in vacuo.

Equation $(A-43\}$ is a difficult transcendenti, juation to solve for a compressible fluid. If, however,

$$
\left(\frac{\Omega S}{\pi c m}\right)^{2} \ll 1
$$

then from $(A-38)$ and $(A-42)$ it can be seen that $\Phi$ is no longer a function of $\Omega$ and tine calculation of the natural frequencies is reduced simply to the computation of the connected mass factor $\$$. The equations of motion of the shell (A-33a,b; $A-41)$ can be employed with the use of $\Phi$ to describe the shell motion independent of the fluid.

The inequality of (8) is met for an incompressible fluid since $c+\infty$. Thus, for incompressible fluids the effective mass is easier to calculate. To determine when a fluid can be considered incompressible the inequality of (8) can be used. A supplementary criteria can be detemined using the coefficient before $R(r)$ in (A-37)

$$
\left(\frac{\pi m r}{s}\right)^{2}-\left(\frac{\Omega r}{c}\right)^{2}+n^{2}
$$


With $c+\infty$ this reduces to

$$
\frac{\pi^{2} m^{2} r^{2}}{s^{2}}+n^{2}
$$

Comparing (A-44a) and $(A-44 b)$ it is evident that the liquid behaves as an incompressible medium on the condition that

$$
\frac{\Omega^{2} b^{2}}{c^{2}\left(n^{2}+\frac{\pi^{2} m^{2} b^{2}}{s^{2}}\right)} \ll 1
$$

Therefore either (7) or (8) can be used to determine whether the fluid should be considered incompressible.

\section{REFERENCES FOR APPENDIX A}

A-1. Kraus, H., Thin Elastic Shel1s, Wiley \& Sons; Inc., New York, (1967).

A-2. Leissa, A. W., "Vibration of Shells," National Aeronautics and Space Administration Report NASA SP-288, (1973).

A-3. Johnson, D. E. and Greif, R., "Dynamic Response of a Cylindrical She11: Two Numerical Methods": AIAA Journal, Vol. 4, No. 3, (1966) pp. 486-494.

A-4. Sanders, 3. L., Jr., "An Improved First-Approximation Theory for Thin Shells", NASA Rept. 24, (1959).

A-5. Landau, L. D. and Lifshitz, E. M., Fluid Mechanics, Addison-Wesley, (1959).

A-6. Cumings, G. and Feng, W., "Modeling Considerations in the Vibration Analys is of Reactor Internals", Lawrence Livermore Laboratory Report UCRL-52023, (Feb. 1976).

A-7. Whev, Y. and Pertsev, A., "Hydro-Elasticity of Shells", Foreign Technology Division, Wright-Patterson Air Fo'ce Base, Ohio, RPT. FTO-MT-24-119-71, (Aug. J971).

A-8. Bletch, H. and Baron, M., "Free and Forced Vibrations of an Infinitely Long Cylindrical Sheî in an Infinite Acoustic Medium," J. Applied Mechanics, Vol. 76, (1954), pp. 167-177. 
APPEADIX B - DEVELOPMENT OF EQUATIONS USED FOR COMPUTATION

In this appendix, we convert the governing equations developed in Appendix $A$ into the difference equations we will use for numerical computation in the computer code SHELVIB.

We wi:: start by casting the shell equations of motion, equations (?) into matrix form. Another matrix equation is developed to describe the she 11 end conditions and both are discretized. The difference form of these equations presents a tridiagonal matrix problem which is soived in a standard manner.

We next will discretize the equation describing the fluid (6). An odd-even cyclic reduction technique used to solve its discretized form will then be described. Finally, the capacitance matrix technique used to handle the mixed boundary value problem on the outer fluid boundary will be discussed.

\section{BASIC STRATEGY}

The basic strategy of the calculational method is to solve the finite difference representations of the shell equations of motion and fluid equation separately but account for the interaction between the two by using the adhesion boundary conditons (9) at the shell-fluid interface. The shell displacements as a function of time for each Fourier mode are determined separately and must be summed to get the total displacement. The forcing function, whether it is applied at the outer boundary of the fluid or on the shell must be expanded in a Fourier series with the appropriate Fourier term applied for each mode calculated. Alternatively, the natural frequencies for each mode can be calculated by inputting a step forcing function of short rise time or a harmonic forcing function and varying its frequency. 
Figure $B-1$ is a flow chart of the computational process for an individual Fourier mode. If the forcing function is specified as a pressure on the outer boundary of the fluid then the calculation is started by solving the fluid equations to determine the pressure distribution in the fluid and at the fluidshell interface. This interface pressure is then input to the shell equations as a radial load from which the displacement and acceleration of the shell are calculated. The radial acceleration of the shell is then used to specify a boundary condition at the fluid-shell interface and new pressure distributions in the fluid and on the shell surface aredetermined from the fluid equations. The average pressure between the new and old time steps is then used as a loading input to the shell equations to determine the shell displacement and acceleration at the next time step. This process is repeated until completion of the problem. The use of the average pressure gave the best convergence compared to other weighting criteria.

\section{SHELL EQUATIONS}

The technique used to solve the shell equations of motion is similiar to that used by Johnson and Greif $[B-1]$. Me start by casting equations (2) into a single matrix equation with dependent variable $z$, a four component column vector. Although we are talking about the nth mode, we will drop the subscript $\mathbf{n}$ for the sake of clarity.

$$
\mathrm{Kz}^{\prime \prime}+\mathrm{Fz}^{\prime}+\mathrm{Gz}=y+\mathrm{Dz}_{z}+\mathrm{Lz}
$$

where

$$
z=\left[\begin{array}{l}
u \\
v \\
w \\
M_{\xi}
\end{array}\right] \quad y=\left[\begin{array}{l}
0 \\
0 \\
q \\
0
\end{array}\right] \quad D=\left[\begin{array}{llll}
1 & 0 & 0 & 0 \\
0 & 1 & 0 & 0 \\
0 & 0 & 1 & 0 \\
0 & 0 & 0 & 0
\end{array}\right]
$$




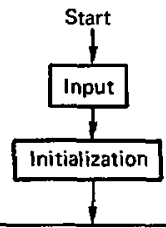

Specify loading on shell wall or pressure on outer fluid boundary and compute pressure distribution on shell wall at old time $\mathbf{j}$

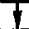

Calculate shell displacements $u_{n}, v_{n}, w_{n}$, bending moment, $\mathrm{M} ;$; and radial acceleration, ${ }^{2}{ }^{2} w_{n} / \partial \tau^{2}$, at old time $j$ (nondimensional)

\section{1}

Calculate fluid boundary conditions at the shell wall for inner and outer fluids at old time j (pressure gradient used as boundary condition is calculated in dimensional terms).

$$
\left(\partial p_{n} /\left.\partial r\right|_{r=a}\right)_{i}= \pm \frac{a_{o} \rho_{f}}{a \rho_{s}} \partial^{2} w_{n} / \partial \tau^{2}
$$

Calculare pressure in fluid regions and on shell wall at new time, $\left(p_{n}\right)_{i+1}$

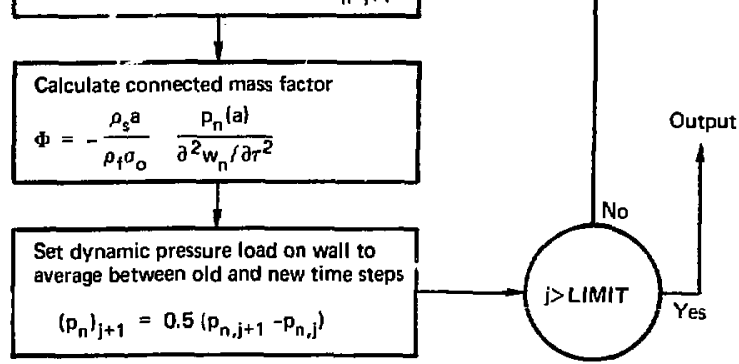

Fig. B-1 Flow chart of calculation for the nth Fourier Mode using the code SHELV1B. 


$$
\begin{array}{lll}
K=\left[\begin{array}{llll}
\alpha_{1} & 0 & 0 & 0 \\
0 & \alpha_{6} & a_{8} & 0 \\
0 & \alpha_{12} & \alpha_{14} & \alpha_{16} \\
0 & 0 & \alpha_{19} & 0
\end{array}\right] & F=\left[\begin{array}{llll}
0 & \alpha_{3} & \alpha_{4} & 0 \\
\alpha_{5} & 0 & 0 & 0 \\
\alpha_{11} & 0 & 0 & 0 \\
0 & 0 & 0 & 0
\end{array}\right] \\
G=\left[\begin{array}{llll}
\alpha_{2} & 0 & 0 & 0 \\
0 & \alpha_{7} & \alpha_{9} & \alpha_{10} \\
0 & \alpha_{13} & \alpha_{15} & \alpha_{17} \\
0 & \alpha_{18} & a_{20} & \alpha_{21}
\end{array}\right] & L=\left[\begin{array}{llll}
c & 0 & 0 & 0 \\
0 & c & 0 & 0 \\
0 & 0 & c & 0 \\
0 & 0 & 0 & 0
\end{array}\right]
\end{array}
$$

The coefficients from equations (2), $\alpha_{1}$ through $\alpha_{21}$, are listed in Table A-1. C is a dimensionless damping coefficient. As before, primes stand for derivatives with respect to $\xi$, the axial space variable and dots, for derivatives with respect to $\tau$, the dimensioniess time variable.

He now wish to cast the end condition into a non-dimensional matrix form in terms of vector 2 . First the variables describing the natural boundary conditions must be expanded in a Fourier series. Ther relationships between the Fourier coefficients of these variables and the components of $z$ must be established. This was done in Appendix A giving

$$
\begin{aligned}
& N_{\xi}=B_{1} u^{\prime}+B_{2} v+B_{3} W^{\prime} \\
& \hat{N}_{\xi \theta}=B_{4} u+B_{5} v^{\prime}+B_{6} W^{\prime} \\
& \hat{Q}_{\xi}=B_{7} u+B_{8} v^{\prime}+B_{9} W^{\prime}+B_{10} W^{\prime} \\
& \theta_{\xi}=-W^{\prime}
\end{aligned}
$$

Aga in the $n$ subscript has been dropped for clarity. The values for $B_{1}$ through $B_{10}$ are 1 isted in Table $A-2$.

Note that the end conditions can be written in the form:

$$
\hat{\Omega} \bar{x}+\Lambda z=\ell
$$


with $\hat{\Omega}, \Lambda$ diagonal matrices and $\ell$ a column vector dictated by the actual end conditions. We can also write (A-21) in a matrix form as

$$
\overrightarrow{\mathrm{x}}=\mathrm{Hz}^{\prime}+\mathrm{Jz}
$$

$$
H=\left[\begin{array}{cccc}
B & 0 & 0 & 0 \\
0 & \beta_{5} & \beta_{6} & 0 \\
0 & B_{8} & B_{9} & B_{70} \\
0 & 0 & -1 & 0
\end{array}\right] \quad J=\left[\begin{array}{llll}
0 & \beta_{2} & \beta_{3} & 0 \\
\beta_{4} & 0 & 0 & 0 \\
\beta_{7} & 0 & 0 & 0 \\
0 & 0 & 0 & 0
\end{array}\right]
$$

Combining (B-2) and (B-3) we obtain the desired form of the equation describing the end conditions

$$
\text { or } \begin{aligned}
& \hat{\Omega} \mathrm{Hz}^{\prime}+(\Lambda+\hat{\Omega} J) z=\ell \\
& \hat{\Omega} \mathrm{Hz}^{\prime}+(I-\hat{\Omega}+\hat{\Omega} J) z=\ell
\end{aligned}
$$

with I the identity matrix.

As an example, for a fixed boundary condition $u=v=w=\theta_{\xi}=0$.

This defines $\hat{\Omega}, \Lambda$, and $\ell$ as

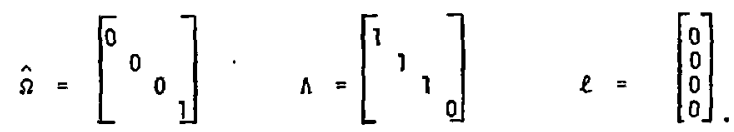

For a simply -supported boundary condition $v=w=M_{E}=N_{\xi}=0$ giving

$$
\hat{\Omega}=\left[\begin{array}{lll}
1 & & \\
& 0 & \\
& 0 & \\
& & 0
\end{array}\right] \quad \Lambda=\left[\begin{array}{lll}
0 & & \\
& 0 & \\
& 0 &
\end{array}\right] \quad \ell=\left[\begin{array}{l}
0 \\
0 \\
0 \\
0
\end{array}\right] .
$$


As a final example, for a free boundary, $N_{E}=\hat{A}_{\xi \theta}=Q_{\xi}=M_{\xi}=0$ for which we have

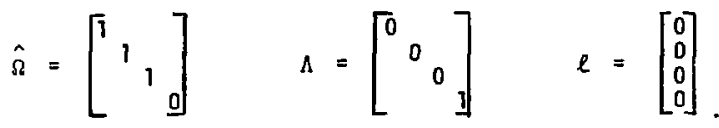

\section{Differencing Scheme}

We now have (10) and (11) describing the shell equations of motion and the end conditions in terms of the Fourier coefficients of the dependent variables, $u, v, w, M_{E}$. We will formulate the differencing schemes and substitute these int: (10) and (11) to produce three algebraic matrix equations which form a tridtagonal systen and can be solved by Gaussian elimination techniques.

We start by defining space and time increments as

$$
\begin{array}{rlrl}
\xi_{i} & =i \Delta & \tau_{j} & =j \varepsilon \\
i & =0,1,2, \ldots \ldots N & j & =-2,-1,0,1,2 \ldots \ldots \\
\Delta=s / a N & \varepsilon=\left(\frac{E}{\rho_{s}}\right)^{1 / 2} \frac{t_{i, j+1}-t_{i, j}}{a}
\end{array}
$$

where the mesh is shown in Figure B-2a.

At positions $\xi_{i}$ and time $\tau_{j}$ we use for derivatives with respect to $\xi_{j}$,

$$
\begin{aligned}
& z^{\prime \prime}{ }_{i, j}=\frac{\left(z_{i+1, j, j}-2 z_{i, j}+z_{i-1, j}\right)}{\Delta^{2}} \\
& z_{i, j}^{\prime}=\frac{\left(z_{i+1, j^{-z} i-1, j}\right)}{2 \Delta}
\end{aligned}
$$




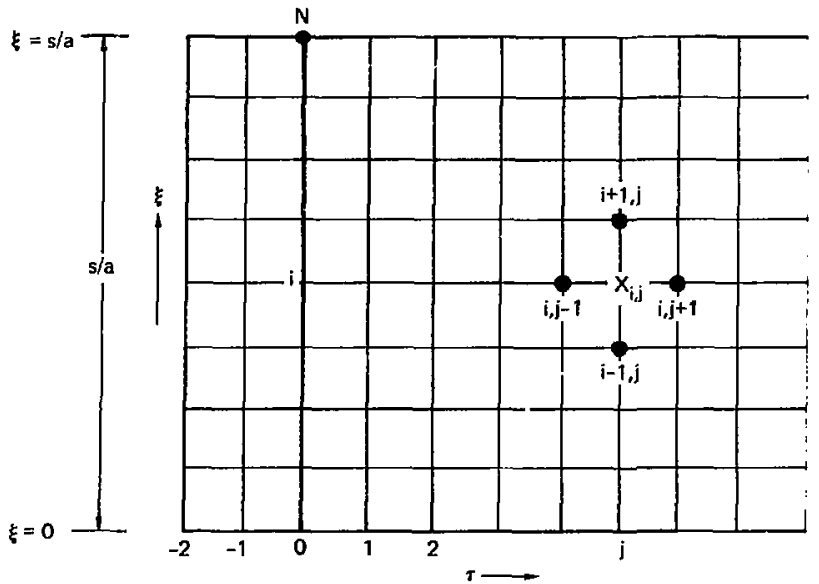

Fig B-2a Mesh for finite differencing of shell equations $(\mathrm{B}-10 \mathrm{a}, \mathrm{b}, \mathrm{c})$.
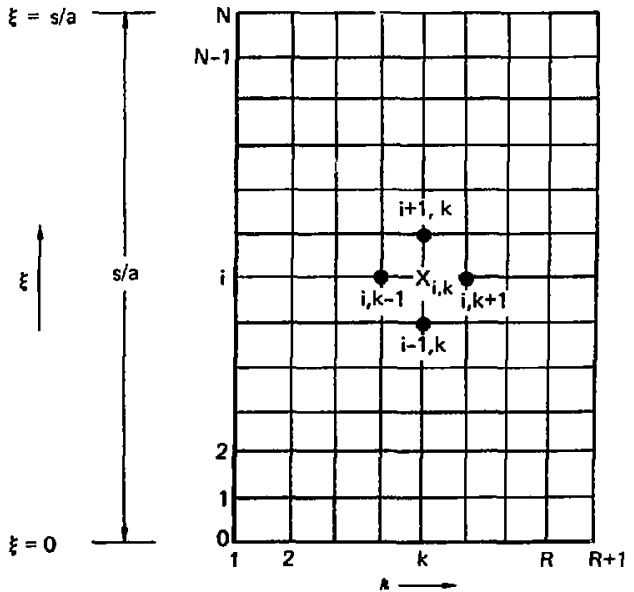

FigB-2b Mesh for finite differencing of fluid equation (a-12a). 
and for $1=0$ and $N$,

$$
\begin{aligned}
& z_{0, j}^{\prime}=\frac{\left(-3 / 2 z_{0, j}+2 z_{1, j}-1 / 2 z_{2, j}\right)}{\Delta} \\
& z_{N, j}^{\prime}=\frac{\left(3 / 2 z_{N, j}-2 z_{N-1, j}+1 / 2 z_{N-2, j}\right)}{\Delta}
\end{aligned}
$$

The backward differencing scheme used to represent the derivatives at the shell ends retains the accuracy of the central differencing used at the other space stations without involying the fictitious space stations at -1 and $N+1$, If we define

$$
z(\xi)=a_{1}+a_{2} \xi+a_{3} \xi^{2}
$$

then we have

$$
\begin{aligned}
& z_{0, j}=z(0)=a_{1} \\
& z_{1, j}=z(\Delta)=a_{1}+a_{2} \Delta+a_{3} \Delta^{2} \\
& z_{2, j}=z(2 \Delta)=a_{1}+2 a_{2} \Delta+4 a_{3} \Delta^{2}
\end{aligned}
$$

if we solve for $a_{2}$ and $a_{3}$ we obtain

$$
a_{1}=\frac{z_{0, j}-2 z 1, j+z, j}{2 \Delta^{2}}, \quad a_{2}=\frac{2 z 1_{2} k^{-3 / 2 z} 0, j^{-1 / 2 z} 2, j}{\Delta}
$$

and since

$$
z^{\prime}(\xi)=\frac{\partial}{\partial \xi}\left(a_{1}+a_{2} \xi+a_{3} \xi^{2}\right)=a_{2}+2 a_{3} \xi
$$

we get

$$
z_{B, j}^{\prime}=z^{\prime}(0)=a_{2}=\frac{\left(-3 / 2 z_{0, j}+2 z_{1, j}-1 / 2 z_{2, j}\right)}{\Delta}
$$

Equation (B-5d) can be obtained in a similar manner. 
For the time derivatives we use,

$$
\begin{array}{r}
\ddot{z}_{i, j}=\bar{\alpha}_{j} z_{i, j}+\bar{\beta}_{j} z_{i, j-1}+\bar{\gamma}_{j} z_{i, j-2}+\bar{\delta}_{i, j-3} \\
(j=0,1,2, \ldots) \\
\dot{z}_{i, j}=\bar{k}_{j} z_{i, j}+\bar{\lambda}_{j} z_{i, j-1}+\bar{\mu}_{j} z_{i, j-2}+\bar{v}_{j} z_{i, j-3 .}
\end{array}
$$

Since we are interested in the forced vibration problem we are assuming $\dot{z}_{i, 0}=\dot{z}_{i, 0}=\ddot{z}_{i, 0} \equiv 0$ although the method couid be extended to include non zero initial conditions. The coefficients for $\left(B-\kappa_{d}, b\right)$ are listed in Table $8 \pi$ ].

Equations ( $B-6 a, b)$ are based on the Houbolt method of numerical integration [B-2] which employes a third degree polynominal in $t$ fitted through four discrete paints $\tau_{j}, \tau_{j-1}, \tau_{j+2}$, and $\tau_{j+3}$. This gives an implicit, stable system of equations with the time increment $\varepsilon$ chosen to give the desired accuracy. As $\varepsilon+\infty$ the solution approaches the correct steady state value. However, the solution is damped if $\varepsilon$ is greater than 1/50 the natural period of the system. Also, this technique must be used correctly during the initial stages of the calculation requiring different values for the coefficients when $j=0,1,2$. These values are given in Table $B-1$.

To get Houbolt's equations we start with

$$
z(\tau)=b_{1}+b_{2} \tau+b_{3} \tau^{2}+b_{4} \tau^{3} .
$$

Then we evaluate using

$$
\begin{aligned}
& z_{i, 1}=z(\varepsilon)=b_{1}+b_{2} \varepsilon+b_{3} \varepsilon^{2}+b_{4} \varepsilon^{3} \\
& z_{i, 0}=z(0)=b_{1} \\
& z_{1,-1}=z(-\varepsilon)=b_{1}-b_{2} \varepsilon+b_{3} \varepsilon^{2}-b_{4} \varepsilon^{2} \\
& z_{1,-2}=z(-2 \varepsilon)=b_{1}-2 b_{2} \varepsilon+4 b_{3} \varepsilon^{2}-8 b_{4} \varepsilon^{3} .
\end{aligned}
$$


Solving for $b_{2}$ and $b_{3}$ in terms of $z_{i}, 1, z_{1}, 0, z_{i},-1, z_{i},-2$, gives

$$
\begin{aligned}
& b_{2}=\frac{1}{6 \varepsilon}\left(2 z_{i, 1}+3 z_{i, 0}-6 z_{i,-1}+z_{i,-2}\right) \\
& b_{3}=\frac{z_{i, 1}-2 z_{i, 0}+z_{i,-1}}{2 \varepsilon^{2}}
\end{aligned}
$$

Now we note that

$$
\begin{aligned}
& \dot{z}(\tau)=b_{2}+2 b_{3} \tau+3 b_{4} \tau^{2} \\
& \ddot{z}()=2 b_{3}+6 b_{4} \tau
\end{aligned}
$$

and so we have

$$
\begin{aligned}
& \dot{z}_{i, 0}=\dot{z}(0)=b_{2}=\frac{1}{6 \varepsilon}\left(2 z_{i, 1}+3 z_{i, 0}-6 z_{i,-1}+z_{i,-2}\right) \\
& \ddot{z}_{i, 0}=\ddot{z}(0)=2 b_{3}=\frac{z_{i, 1}-2 z_{i, 0}+z_{i,-1}}{\varepsilon^{2}}
\end{aligned}
$$

If we now use the initial conditions that $z_{i, 0}=\dot{z}_{i, 0}=\ddot{z}_{i, 0}=0$ we find that

$$
z_{i, 1}=-z_{i,-1} \text { and } z_{i,-2}=-8 z_{i, 1} \text {. }
$$

Next if we solye for $b_{2}$ and $b_{3}$ of $(B-7)$ in terms of $z_{i, j}, z_{i, j-1}, z_{i, j-2}$, $z_{i, j-3}$ we get the standard form of the Houbolt equations

$$
\begin{aligned}
& \dot{z}_{i, j}=\frac{1}{6 E}\left(11 z_{i, j}-18 z_{i, j-1}+9 z_{i, j-2} 2 z_{i, j-3}\right) \\
& \ddot{z}_{i, j}=\frac{1}{\varepsilon^{2}}\left(2 z_{i, j}-5 z_{i, j-1}+4 z_{i, j-z}-z_{i, j-3}\right)
\end{aligned}
$$

Substituting $(B-B a, b)$ into $(B-9 a, b)$ gives formulas for the first two time increments $(j=1,2)$ of the calculation in terms of $\ddot{z}_{i, 1}, \dot{z}_{i, 1}$, $z_{i, 1}, z_{i, 2}, i_{i, 2}, z_{i, 2}$ 


$$
\begin{aligned}
& \dot{z}_{i, 1}=\frac{3 z_{i, 1}}{\varepsilon} \quad \ddot{z}_{i, 1}=\frac{6 z_{i, 1}}{\epsilon^{2}} \\
& \dot{z}_{i, 2}=\frac{1}{6 \varepsilon}\left(11 z_{i, 2}-16 z_{i, 1}\right) \quad z_{i, 2}=\frac{1}{\varepsilon^{2}}\left(2 z_{1,2}-4 z_{i, 1}\right) \quad(B-9 e, f)
\end{aligned}
$$

From $(B-9 a, \ldots, f)$ the coefficients for $(B-6 a, b)$ shown in Table $B-1$ are obtained.

If we now substitute (B-5, B-6) into (10) and (11) we get three matrix equations in terms of three unknowns, $z_{i+1, j}, z_{i, j}$, and $z_{i-1, j}$.

$$
\begin{aligned}
& 2_{0, j} z_{2, j}+A_{0, j} z_{1, j}+B_{0, j} z_{0, j}=E_{0, j} \quad(i=0) \\
& A z_{i+1, j}+B_{j} z_{i, j}+C z_{i-1, j}=E_{1, j} \quad(i=1,2, \ldots, N-1) \\
& B_{N, j} z_{N, j}+C_{N, j} z_{N-1, j}+2_{N, j} z_{N-2, j}=E_{N, j} \quad(i=N)
\end{aligned}
$$

with

$$
\begin{aligned}
& A=\frac{2 K}{\Delta}+F \quad A_{0, j}=\frac{2 \hat{\Omega}_{0, j}{ }^{H}}{\Delta} \\
& B_{j}=-\frac{\Delta K}{\Delta}+2 \Delta\left[G-\left(D \bar{\alpha}_{j}+L \bar{K}_{j}\right)\right] \\
& B_{0, j}=-\frac{3 \hat{\Omega}_{0, j} j^{H}}{2 \Delta}+I-\hat{\Omega}_{0, j}+\hat{\Omega}_{0, j}{ }^{J} \\
& B_{N, j}=\frac{3 \hat{\Omega}_{N, j} H^{H}}{2 \Delta}+I-\hat{\Omega}_{N, j}+\hat{\Omega}_{N, j}^{J} \\
& c=\frac{2 K}{\Delta}-F \quad c_{N, j}=-\frac{2 \hat{\Omega}_{N, j} L_{-}^{H}}{\Delta} \\
& E_{i, j}=2 \Delta y_{i, j}+2 \Delta_{L}^{r}\left(D \bar{B}_{j}+L \pi_{j}\right) z_{i, j-1}+\left(D \bar{\gamma}_{j}+L \bar{\mu}_{j}\right) z_{i, j-2} \\
& +\left(D \bar{\delta}_{j}+\left[\bar{v}_{j}\right) z_{j, j}, 3\right]
\end{aligned}
$$




$$
\begin{array}{ll}
E_{0, j}=\ell_{0, j} & E_{N, j}=\ell_{N, j} \\
\hat{O}, j=-\frac{\hat{\Omega}_{0, j^{H}}}{2 \Delta} & \hat{N N, j}=\frac{\hat{\Omega}_{N, j^{H}}}{2 \Delta}
\end{array}
$$

TARE B-1

Formula's for the Coefficients of the Difference

Form of the Time Derivatives, Equation $(B-6 a, b)$

$$
\begin{aligned}
& \bar{\alpha}_{0}=\bar{\beta}_{0}=\bar{Y}_{0}=\bar{\delta}_{0}=0 \\
& \bar{\alpha}_{1}=\frac{6}{E^{2}}, \bar{\beta}_{1}=\bar{\gamma}_{1}=\bar{\delta}_{1}=0 \\
& \bar{\alpha}_{2}=\frac{2}{E^{2}}, \bar{\beta}_{2}=-\frac{4}{\varepsilon^{2}}, \bar{Y}_{2}=\bar{\delta}_{2}=0 \\
& \bar{\alpha}_{j}=\frac{2}{\varepsilon^{2}}, \bar{\beta}_{j}=-\frac{5}{\varepsilon^{2}}, \bar{Y}_{j}=\frac{4}{\varepsilon^{2}}, \bar{\delta}_{j}=-\frac{1}{E^{2}} \\
& (j=3,4, \ldots . .)
\end{aligned}
$$

$$
\begin{aligned}
& \bar{\kappa}_{0}=\bar{\lambda}_{0}=\bar{\nu}_{0}=\bar{v}_{0}=0 \\
& \bar{\kappa}_{1}=\frac{3}{\varepsilon}, \bar{\lambda}_{1}=\bar{\nu}_{1}=\bar{v}_{1}=0 \\
& \bar{k}_{2}=\frac{11}{6 \varepsilon}, \bar{\lambda}_{2}=-\frac{8}{3 \varepsilon}, \bar{\mu}_{2}=\bar{v}_{2}=0 \\
& \bar{\kappa}_{\mathbf{j}}=\frac{11}{6 \varepsilon}, \bar{\lambda}_{\mathbf{j}}=-\frac{3}{\varepsilon}, \bar{\mu}_{\mathbf{j}}=\frac{3}{2 \varepsilon}, \bar{v}_{\mathbf{j}}=-\frac{1}{3 \varepsilon}
\end{aligned}
$$

$$
(j=3,4, \ldots \ldots)
$$

$-75-$ 
Solution Method

To solve equations $(B-10 a, b, c)$ at any time $j$, a Gaussian elimination technique is used. First a recurrence relation is formed relating $\mathbf{z}_{\mathbf{i}, \mathbf{j}}$ and $z_{i-1, j}$. Then using the boundary condition at $i=N$ equation (B-10C) together with this recursion formula is used to calculate $z_{\mathrm{N}-1, j}$, etc., down to $z_{0, j}$.

Assume as the recurrence relation

$$
\begin{gathered}
z_{i, j}=-P_{i, j} z_{i+1, j}+x_{i, j} . \\
(i=1,2,3, \ldots \ldots, N-1)
\end{gathered}
$$

(B-11a)

Then from equations $(B-10 a)$ and $(B-10 b)$ we get

$$
\begin{aligned}
& P_{l, j}=\left[B_{0, j} c^{-1} B_{j}-A_{0, j}\right]^{-1}\left[B_{0, j} C^{-1} A-Q_{0, j}\right] \\
& x_{l, j}=\left[B_{0, j} c^{-1} B_{j}-A_{0, j}\right]^{-1}\left[B_{0, j} c^{-1} E_{1, j}-E_{0, j}\right] .
\end{aligned}
$$

Now from equation (B-11a) we have

$$
z_{i-1, j}=-p_{i-1, j} z_{i, j}+x_{i-1, j}
$$

and if this is substituted into equation (B-10b) we get

$$
\begin{aligned}
& P_{i, j}=\left[B_{j}-C P_{i-1, j}\right]^{-1} \quad\{i=2,3, \ldots, N-1) \\
& x_{j, j}=\left[\begin{array}{lll}
B_{j} & -C P_{i-1, j}
\end{array}\right]^{-1}\left[E_{i, j}-C x_{i-1, j}\right] .
\end{aligned}
$$

If we use equation (8-11a) for $z_{N-1, j}$ and $z_{N-2, j}$ we get the desired equation for $z_{N, j}$

$-76-$ 


$$
\begin{aligned}
z_{N, j}=- & {\left[B_{N, j}-c_{N, j} P_{N-1, j}+Q_{N, j} P_{N-2, j} P_{N-1, j}\right]^{-1} } \\
& \cdot\left[c_{N, j} x_{N-1, j}-2_{N, j} P_{N-2, j} x_{N-1, j}+2_{N, j} x_{N-2, j}\right. \\
& \left.-E_{N, j}\right]
\end{aligned}
$$

We then calculate down from $z_{N, j}$ to get all $z_{i, j}$ until we end with

$$
z_{0, j}=C^{-1}\left[E_{1, j}-A z_{2, j}-B_{j} z_{1, j}\right]
$$

The solution : or the $z$ 's involves only irversions of $4 \times 4$ matrices. It should be noted that if the baundary conditions at the end $i=0$ and equation (B-10a) are used to caiculate $z_{0, j}$ instead of equation (B-11c) difficulty may arise since the matrix $B_{0, j}$ may be singular.

\section{FLUID EQUATION}

The equation used to represent the $n$th circumferential mode of the fluid is (6). This equation is elliptic in $\xi, n$ cylindrical coordinates and is used to represent the fluid both inside and outside the shel1. The outer fluid region represents a special problem since we want to be able to specify mixed boundary values on its outer boundary. In this way we can model a rigid outer wall over most of the boundary $(\partial \mathrm{p} / \partial r=0)$ and simulate a pump inlet port over a small portion $(p=p(\tau))$ if we wish.

A fast, direct solution method [B-3] was employed to handle the fluid equations with suitable modification to handie the mixed boundary values. This direct method takes advantage of the block structure of the finite difference form of (6) and employes an odd-even cyclic reduction technique to directly solve the equation. Equation (6) written in a slightly different form becomes 


$$
\frac{1}{r} \frac{\partial}{\partial r}\left(r \frac{\partial p_{n}}{\partial r}\right)+\frac{\partial^{2} p_{n}}{\partial \xi^{2}}-\frac{n^{2}}{r^{2}} p_{n}=0 .
$$

Differencing Scheme

The dimensionless finite difference form of (6) employes centered difference approximations to the derivatives at a grid of points $\left(\xi_{j}, n_{k}\right)$ as shown in Fig. B-2b.

$$
\begin{aligned}
& \xi_{i}=i \Delta \\
& r_{k}=r_{1}+\frac{(k-1)}{R}\left(n_{R+1}-r_{1}\right) \\
& i=0,1,2, \ldots, N \\
& k=1,2, \ldots, R+1 \\
& \Delta=s / a N
\end{aligned}
$$

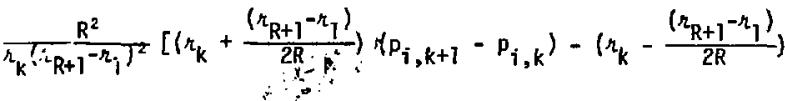

$$
\begin{aligned}
& \left.-\left(p_{i, k}-p_{i, k-1}\right)\right]+\frac{1}{\Delta^{2}}\left[p_{i-1, k}-2 p_{i, k}+p_{i+1, k}\right] \\
& -\frac{n^{2}}{r_{k}} p_{i, k}=0
\end{aligned}
$$

For the fluid inside the shell we have $\left.\frac{\partial p}{\partial r}\right]_{i, r_{0} / a}=0$ on the inner boundary and $\left.\frac{\partial p}{\partial r}\right]_{i, 1}=\frac{a}{h} \frac{\rho_{f}}{\rho_{s}} \ddot{w}$ on the outer boundary as per $(A-32 a)$. Since along the shell wall the solution $p_{i, R+l}$ is unknown and only the derivative is defined at the outer boundary, we must substitute the central difference approximation

$$
p_{1, R+2}=p_{1, R}+2 \frac{n_{R+1}-n_{1}}{R} \frac{a}{h} \frac{\rho_{f}}{\rho_{S}} \ddot{H}
$$


in to ( $\mathrm{B}-12 \mathrm{a})$ to obtain

$$
\begin{aligned}
& \frac{2 R^{2}}{\left(r_{R+1}^{-r_{1}}\right)^{2}} P_{i, R}-\frac{2 R^{2}}{\left(r_{R+1}{ }^{-r}\right)^{2}} p_{i, R+1}+\frac{1}{\Delta^{2}}\left[p_{i-1, R+1}-2 p_{i, R+1}+p_{i+1, R+1}\right] \\
& -\frac{n^{2}}{n_{R+1}^{2}} P_{i, R+1}=-\frac{(2 R+1) r_{R+1}-r_{1}}{\left(r_{R+1}{ }^{-n}\right)_{R+1}} \frac{a}{h} \frac{\rho_{f}}{\rho_{S}} \ddot{w}
\end{aligned}
$$

as a representation of $(B-12 a)$ on the boundary ${\gamma_{R+1}}^{*}$ In this way we do not have to use the fictitious points $\left(\xi_{j}, r_{R+2}\right)$. A similar technique is used to arrive at an equation representing the boundary points $\left(\xi_{i}, r_{l}\right)$ without involying the fictitious points $\left(\xi_{i}, n_{0}\right)$.

$$
\begin{aligned}
& \frac{2 R^{2}}{\left(n_{R+1}-1_{1}\right)^{2}} P_{i, 2}-\frac{2 R^{2}}{\left(n_{R+1}{ }^{-r}\right)^{2}} P_{i, 1}+\frac{1}{\Delta^{2}}\left[P_{i-1,1}-2 P_{i, 1}+P_{i+1,1}\right] \\
& -\frac{n^{2}}{n_{1}^{2}} P_{i, 1}=\frac{(2 R+1) n_{1}-r_{R+1}}{\left(r_{R+1}\right)^{\left.-n_{1}\right) n_{1}}} \frac{a}{h} \frac{\rho_{f}}{\rho_{s}} \ddot{w}
\end{aligned}
$$

For the fluid outside the shell the no-slip boundary condition is applied at the inner boundary as per (A-32b). The equation developed for the points on the shell wall is similiar to $(B-12 \sigma)$. The treatment of the outer boundary involves mixed boundary values and will be described later.

Both Dirichlet, $p(0, n)=0, p(s / a, n)=0$ or Neumann boundary conditions, $\frac{\partial p(0, r)}{\partial \xi}=\frac{\partial p(s / a, r)}{\partial \xi}=0$ can se specified on the ends of both fluid regions. The boundary points for the Meumann condition are handled in a manner so that values at the $i=-I, N+1$ points need not be specified. This is done in a manner similar to what we did for the radial boundaries. 
Dirichlet boundary conditions : :re used for 211 calculations in this report. It was interesting to note that both the analytical solution, which was for an infinitely long region, and the experimental results approached the computer code solution with Dirichlet boundary conditions on both ends of the fluid regions.

\section{Solution Hethod}

The solution method for solying (B-12) was develoned by Swa rztrauber and sweet $[\mathrm{B}-3]$ and is a direct, non-iterative method which can be used on the general class of elliptic partial differential equations in two dimensional geometry. The solution or the derivative of the solution can be specified at either radial boundary and at either end, Also a periodic boundary condition, i.e. $P_{a, \pi}=P_{N, \pi}$, can be applied on the ends, We have modified Swarztrauber's and Sweet's solution method to allow for specification of the solution and the derivative of the solution together on the outer radial boundary by use of a caparitance matrix technique [8-4].

The direct solution method for (B-12) takes advantage of the block structure of this set of finite difference equations. We first put then in the form

$$
\begin{array}{r}
a_{k} p_{i, k-1}+b_{k} p_{1, k}+c_{k} p_{i, k+1}+p_{i-1, k}-2 p_{i, k} \\
+p_{i+1, k}=0
\end{array}
$$

and this system is solved oy the Buneman [B-5] variant of cjclic reduction. This involves construcing a $R \times R$ matrix from the $a_{k}, b_{k}, c_{k}$, coefficients and employing this matrix, which is block tri-diagonal, to reduce the number 0 . variables by two, in steps, until a single equation is left. This equation is 
then solved and its solution used to obtain solutions for all the other unknowns. For this system to work s must be subdivided into $N$ increments such that $N=2^{\tilde{P}} 3^{\tilde{q}} 5^{\tilde{r}}$ where $\tilde{p}, \tilde{q}, \tilde{r}$ are non-negative integers.

Dorr [B-6] has shown that this direct method of solution is significantly faster than the usual iterative techniques, for which, in addition. an appropriate acceleration factur must be determined.

The above method of solution works when one type of boundary valve is allowed per boundary. For the case with mixed boundary values this method was modified using a capacitance matrix technique similar to that described by Buzbee $[B-4]$.

The capacitance matrix technique involves a modification of rows such that the cyclic reduction process can still be used on the modified system. For an elliptic difference system described by the matrix equation

$$
\hat{A p}=\hat{s}
$$

we define a modified matrix $\hat{B}$ with

$$
\hat{B p}=\hat{t}
$$

which can be solved using the cyclic reduction method. To determine $\hat{x}$ we need to know certain elements of $\left(\hat{A}^{-1}\right)$. Since these elements are independent of $\hat{s}$ this computation can be done in a preprocessing phase. Using the results of preprocessing, the solution of (B-14) can be done efficiantiy.

We start by defining:

$\bar{R}=\{1,2, \ldots \ldots, N\}$

$\overline{\mathbf{S}}=\bar{q}$ element sutset of $\bar{R}$ over which $p$ instead of $\partial p / \partial r$ is specified and soive for

$$
g_{i}=\hat{B}^{-1} e_{i} \quad \text { iE } \bar{\xi}
$$


over subset $\vec{S}$ where $\hat{B}$ is the matrix coefficient for equation (B-13) with Neumann boundary conditions in both radial directions and $e_{i}=1.0$. Here $e_{i}$ denotes the $i$ th column of the $N$ by $N$ identity matrix. We now define the unknown for which a solution is desired as

$$
p=\hat{p}+\frac{\sum}{s} \beta_{j} g_{i}
$$

where the $B_{j}$ are yet to be found. For all rows $k$ outside of $\bar{S}$ we can determine $p$ for the $(N-\bar{q})$ unmodified equation of (B-1A) since

$$
(\hat{A} p)_{k}=(\hat{B} p)_{k}=\hat{s}_{K} .
$$

The remaining $\bar{q}$ equations are satisfied by picking the $\beta_{\mathbf{i}}$ properly.

$$
\hat{(A P})_{i}=\hat{s}_{i}=(\hat{A P})_{i}+\sum_{s} B_{j}\left(\hat{A} g_{j}\right)_{i}
$$

and with

$$
\hat{C} \equiv\left(\hat{A} g_{j}\right)_{i} \quad \gamma \equiv \hat{s}_{i}-(\hat{A P})_{i}
$$

we obtain the $\beta_{i}$ from the matrix equation

$$
\beta=\hat{c}^{-1} \gamma .
$$

This is called the capacitance system and $\hat{\mathcal{C}}$ the capacitance matrix. 
B-1. Johnson, D. and Greif, R., "Dynamic Response of a Cylindrical Shel1: Two Numerical Methods," AIAA Journal, Vol. 4, No. 3, (1966), pp 486-494.

B-2. Houbolt, 3. C., "A Recurrent Matrix Solution for the Dynamic Response of Elastic Aircraft," J. of the Aeronautic Sciences, (Sept. 1950).

B-3. Swarztrauber, P. al.d Sweet, R. , "Efficient Fortran Subprogram for the Solution of Elliptic Partial Differential Equations" NCAR-TN-IA-109. Nat'l Center for Atmospheric Research, Boulder, Colorado (July, 1975).

B-4. Buzbee, B. L., "A Capacitance Matrix Technique", Sparse Matrix Computations Ed. J. R. Bunch \& D. J. Rose, Academic Press, (1976).

B-5. Buneman, 0., "A Compact Non-iterative Poisson Solver," Rep. 294, Stanford University Institute for Plasma Research, Staitord, Calif. (1969).

B-6. Dorr, F. H.: "The Direct Solution of thic Discrete Poisson Equation on it Rectangle," SIAM Review, Vol. 12, Ho. 2, (April 1970). 


\section{APPENDIX C - DESCRIPTJON OF EXPERIMENT}

To check the validity of the assumptions used in formulating the computer code SHELVIB it appeared desirable to check the code against experimental data. For this purpose a thinwalled, circular, cylindrical, steel shell was fabricated and instrumented with dynamic pressure transducers and miniature accelerometers. This shell was placed in a larger, thick walled pipe, called the canister, filled with liquid. The shell was clamped at its top end to the canister. A system was designed to introduce a variable, but single frequency sinusoidal pressure wave onto the shell through ports in the canister. In this way the natural frequencies, damping coefficients, and radial displacements of the shell could be determined and compared to those computed by SHELVIB. The sizing of the shell and canister was such as to approximate a $1 / 12$ scale model of the core barrel of a pressurized water reactor.

\section{DESCRIPTION OF APPARATUS}

The layout of the experimental apparatus is shown in Fig. C-1 and C-2. The canister is bolted to a supporc base which is part of an electromagnetic exciter (max. rating $60 \mathrm{~g}, 2000 \mathrm{~Hz}, 22 \mathrm{kN}$ ). The exciter drives a piston in a $63.5 \mathrm{~mm}$ (2.5 in.) hydraulic cylinder alsi resting on the base. Pressure waves generated by the piston travel up the piping and into either one or both of the inlet ports in the canister. If one port is not in use it is blanked off with blind flanges. Pulse amplitude can be varied up to $\pm 140 \mathrm{kPa}(20 \mathrm{psi})$ at frequencies up to $1000 \mathrm{~Hz}$. 


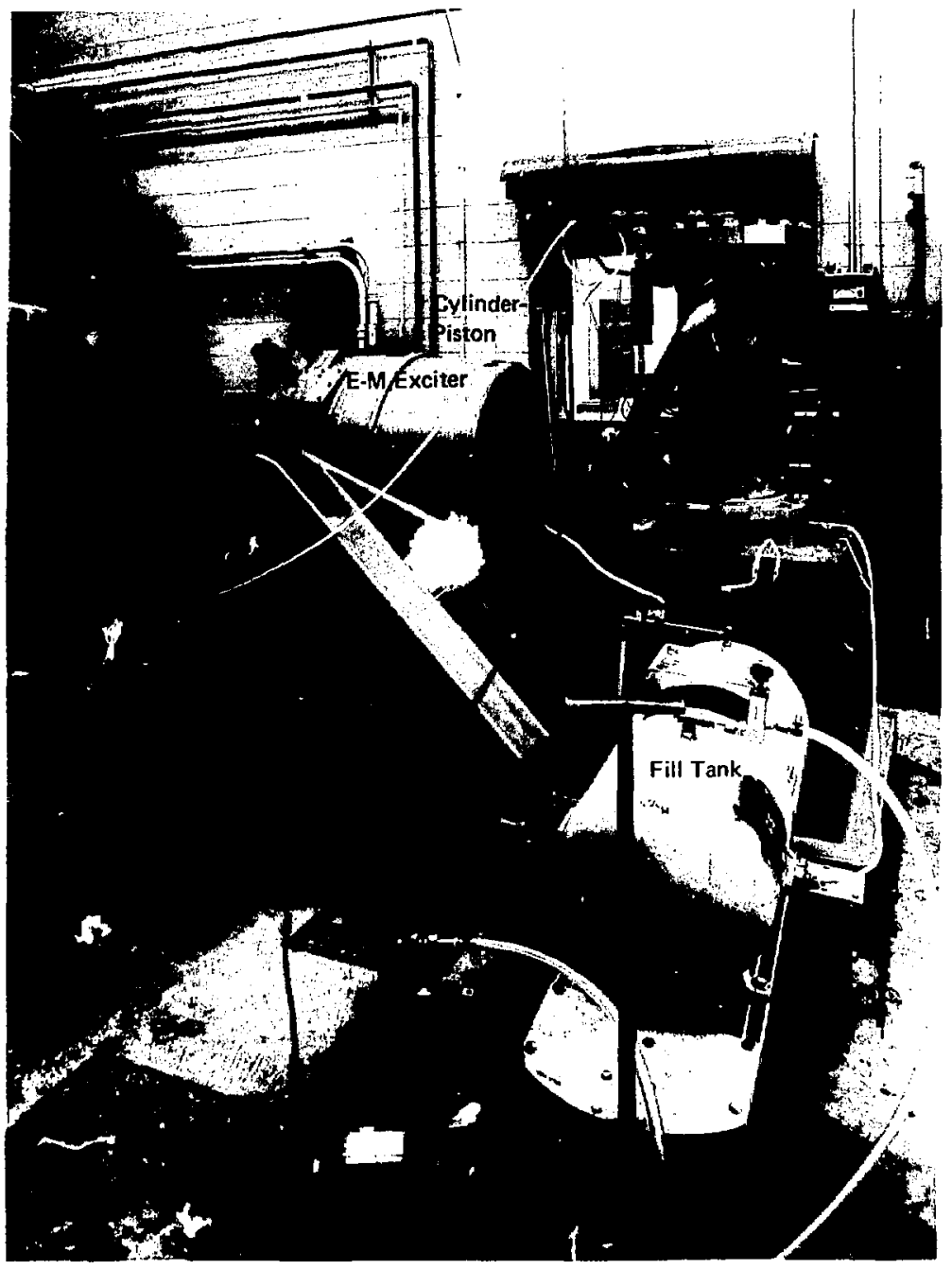

Fig. C.1. Photograph of experimental systems. 

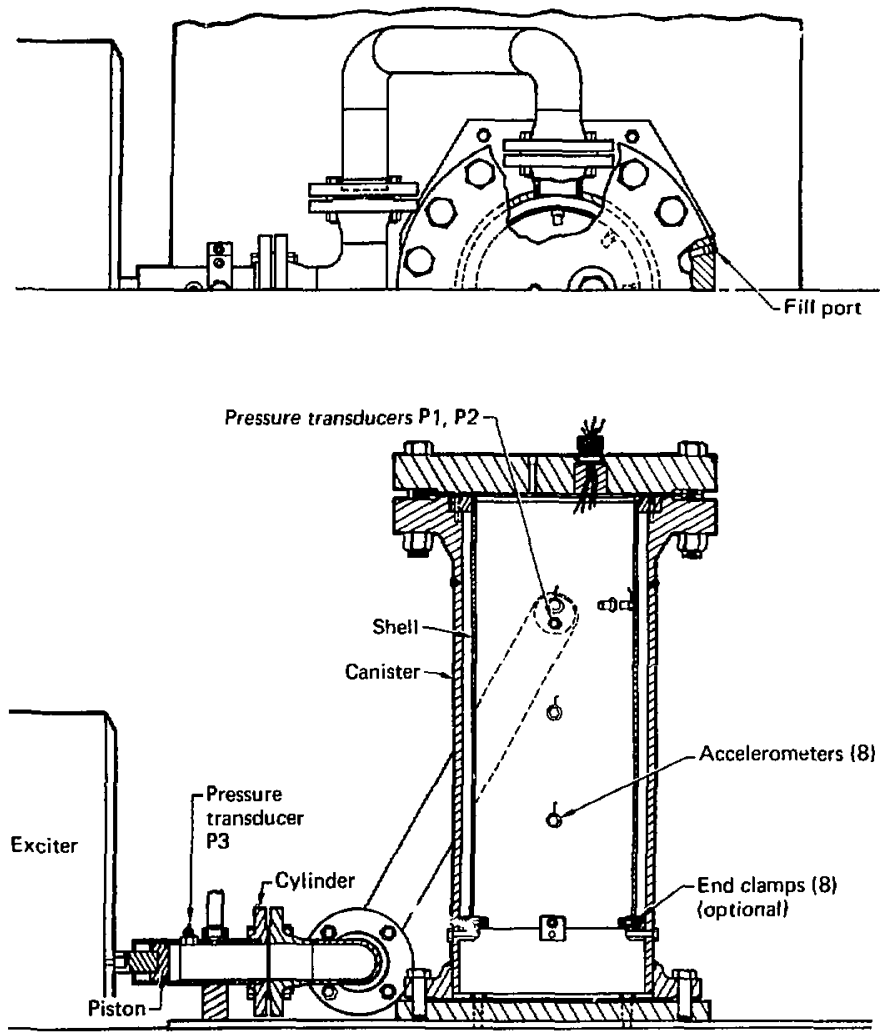

Fig. C-2a Canister-shell layout. 


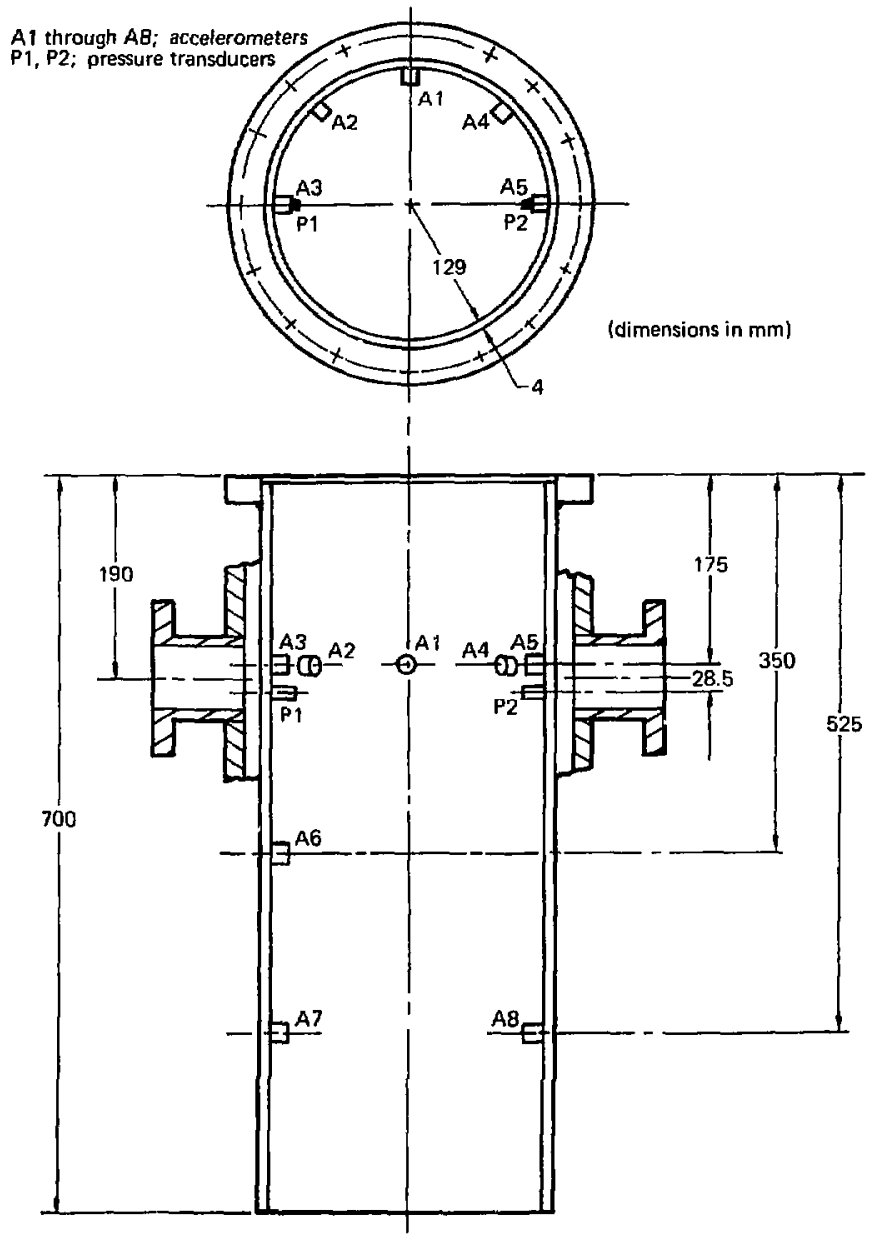

Fig. C-2b instrumented sht:i 
The pressure pulses implnge on the instrumented cylindrical shell inside the canister. The shell is shown in Fig. C-2b. It is held in a clamped condition at the top with sufficient cap screws to approximate a fixed boundary condition and can either be clamped or free at the bottom. Clamping at the bottom is accomplished by means of eight clamps with thumb screws. Since compressibility effects must be minimized to verify the computer code SHELVIB the test vessel is designed to be evacuated before filling with airfree fluid so that the acoustic velocity will be high enough to meet the criteria expressed in equations (7) and (8). The canister wall is thick enough so as to approximate a rigid wall, thus conforming to the boundary conditions which can be simulated in SHELVIB and also maintaining the effective bulk madulus of the water. The important dimensions of the test apparatus are listed in Table $C-1$.

\section{INSTRUMENTATION AND DATA REDUCTION}

Instrumentation consists of eight accelerometers and four pressure transducers. These are read out directly either on a computer-based, transpartab]e, date acquisition and control system (TDAC) [C-1] or a ga?vanometer strip chart recorder. The TOAC system can convert analog signals to digital data for storage, output, or processing. A 16-jit central processor, programmable in FORTRAN, can manipulate the data, e.g. subtract background, integrate acceleration data to get displacement, convert from the time domain to the frequency domain, and manipulate data in the frequency domain. This can all be done on line while data is being collected or at a later date from magnetic tape records. Output can be in the form of plots, printout, or records on magnetic tapes. 
Table C-1 - Dimensions of Experimental Apparatus

\section{Shell}

mean radius, a
wall thickness, $h$
length, $s$
flange radius
flange thickness
material
density, $\rho_{s}$
$E$
$u$

$131 \mathrm{~mm}(5.17 \mathrm{in.})$

$4 \mathrm{~mm}(0.156 \mathrm{in.})$

700 mat (27.50 in.)

$170 \mathrm{~mm}$ (6.63 in.)

$24 \mathrm{~mm}(0.938$ in.)

stainless steel (Type 304 )

$7830 \mathrm{~kg} / \mathrm{m}^{3}\left(0.283 \mathrm{lb} / \mathrm{in}^{3}\right)$

$2.00 \times 10^{-11} \mathrm{~N} / \mathrm{m}^{2}\left(29.0 \times 10^{6} \mathrm{psi}\right)$

0.295

Canister

$\begin{array}{lc}\text { inside radius, b } & 150 \mathrm{~mm}(5.94 \mathrm{in.}) \\ \text { wall thickness } & 11 \mathrm{~mm}(0.44 \mathrm{in.}) \\ \text { gap, b- }(a+h / 2) & 17.5 \mathrm{~mm}(0.69 \mathrm{in.}) \\ \text { inside length } & 815 \mathrm{~mm}(32.13 \mathrm{in.}) \\ \text { volume } & 0.055 \mathrm{~m}^{3}\left(3350 \mathrm{in}^{3}\right) \\ \text { port, dia. } & 63 \mathrm{~mm}(2.47 \mathrm{in.}) \\ \text { port, orientation } & 2-180^{\circ} \text { apart }\end{array}$


A piezoresistive, strain gage accelerometer is used because of its high sensitivity $(10 \mathrm{mV} / \mathrm{g})$ and small size $(28 \mathrm{gms})$. Specifications for the accelerometer are shown in Table $\mathrm{C}-2$. It has a range of $\pm 25 \mathrm{~g}$ and responds within $\pm 5 \%$ over a band width of $0-750 \mathrm{~Hz}$. Shell accelerations are in the range of $0.5 \mathrm{~g}$ to $25 \mathrm{~g}$ for pressure pulses up to $\pm 140 \mathrm{kPa}$. The accelerometers are mounted on the inside of the shell, as shown in Fig. $\mathrm{C}-2 \mathrm{~b}$ to sense radial acceleration exclusively. They are secured to the shell by means of threaded studs. Flats vere machined on the inside surface of the shell to assure a secure mounting.

A quartz, low impedance, pressure transducer is used to sense pressure. It operates over a pressure range of $550 \mathrm{kPa}$ ( $80 \mathrm{psi}$ ) and has a bardwidth of $20 \mathrm{kHz}$. Its active face is mounted flush to the outside diameter of the she11 through a threaded hole from inside the shell. Further specifications are 1 isted in Table $\mathrm{C}-2$.

It was necessary to operate the instrumentation inmersed in the test fluid. A certain amount of difftculty was experienced when water was used because of leakage into the cable connector at the accelerometer housing. The accelerometers and pressure transducers are hermetically sealed units and the cable connectors were sealed on the outside with a silastic compound. Water leakage through the cable bundle sheath traveled dorn inside the bundle causing shoriting between the connector pins over a period of time. However, sufficient data was gathered before the signat became unusable. Leakage was not a problem when insulating transformer oil was used as the test fluid. 
Table C-2 - Instrumentation Specifications

Accelerometer

rated range
sensitivity
natural frequency
frequency response
damping ratio
transverse sensitivity
linearity and hysteresis
excitation
zero output
model
diameter
length
weight

$\pm 25 \mathrm{~g}$

$10 \mathrm{mV} / \mathrm{g}$

$2500 \mathrm{~Hz}$

$\pm 5 \%, 0$ to $750 \mathrm{~Hz}$

0.7 nominal

3\% maximum

$+1 \%$ of reading, maximum,

to $+25 g$

$10 \mathrm{Vdc}$

$\pm 25 \mathrm{mV}$ maximum at $10 \mathrm{~V}$

Endevco Model 226âC-25

$15.5 \mathrm{~mm}$ (0.610 in.)

$25.4 \mathrm{~mm}$ (1.00 in.) $?$

28 gms.

$550 \mathrm{kPa}$ (80 psi)

$5 \mathrm{~Pa}$ (.0008 psi ms)

$100 \mathrm{mV} / \mathrm{ps} 1$

$+1 \%$ full scale

resonant, frequency

$130 \mathrm{kHz}$

vibration sensitivity

$.005 \mathrm{psi} / \mathrm{g}$

frequency response

$\pm 5 \%, 0.05$ to $20,000 \mathrm{~Hz}$

model

Kistler Model 206

diameter

$11 \mathrm{~mm}(0.436$ in.)

length

$33 \mathrm{~mm}$ (1.30 in.)

weight

22 gms 
MEASUREMENT TECHNIQUES

Natural Frequencies and Mode Shapes

The natural frequencies and mode shapes of the instrumented shell were determined using an impact technique and also by sweeping the frequency spectrum using a harmonic exciting source.

The impact technique required that the shell be struck a sharp blow with an instrumented hammer at 26 different locations as shown in Fig. C-3. The acceleration on accelerometer $A 1$ was recorded for each blow as was the impulse itseif from an accelerometer mounted on the hammer. On TDAC the Fourier transform of the accelerometer signal was divided by the Fourier transform of the impulse to give a transfer function, $H(j \omega)$. The inverse transform, $F[H(j \omega)]$, gives the acceleration output to a perfect impulse.

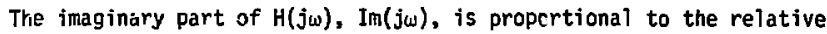
amplitude of the point struck. By comparing the imaginary part for each point at a given frequency, the mode shape can be constructed.

Natural frequencies and modes were also identified by introducing a sinusoldar prsssure wave through inlet forts in the canister and sweeping the frequency spectrum. Resonances were easily identified by observing the amplitude and phase of the accelerometer responses. The prossure waves were generated by a piston-cylinder arrangement with the piston connected to an electro-magnetic exciter. The waves traveled about $0.75 \mathrm{~m}$ to the shell through connecting piping. Peak acceleration of the exciter head $e^{f}$ from 1 to $2 \mathrm{~g}$ was sufficient to give the pressure amplitudes needed. The accelerometer signals at each she!l resonance were recorded using TDAC or strip chart recorders. Plats of these signals were studied to give the phase and amplitude relationship between signals and from this information modes were identified. 


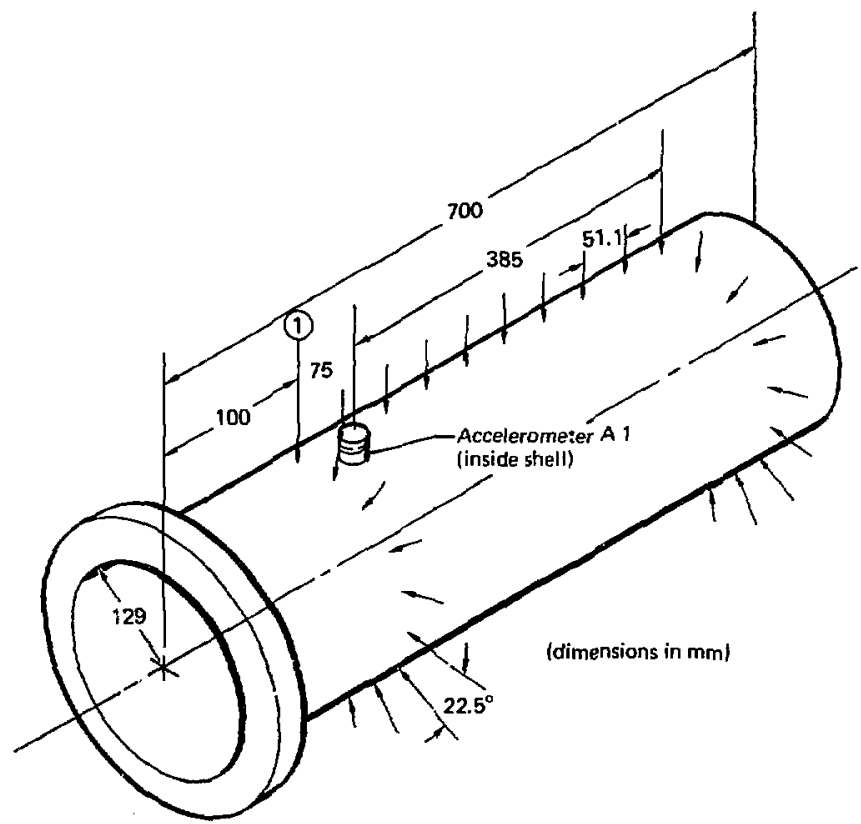

Fig. C-3 impact technique for finding natural frequencies and modes. 
The advantage of the impact technique is that it is quick and only shell resonances are excited. A possible disadvantage is that the top of the canister has to be left open to allow access to the shell. Thus, the fluid remains at atmospheric pressure and with a normal compliment of dissolvedgas. Under these conditions the acoustic velocity is very 10w, e.g. $100 \mathrm{~m} / \mathrm{sec}[\mathrm{C}-2]$, so that the inequalities on which the incumpressibility assumption are based (equations (7) and (8)) become marginally applicable. Since the frequencies measured using the impact technique compared to within 10\% those measured using harmonic excitation this disadvantage was not a concern here.

The advantage of the harmonic excitation technique is that the system is closed and can be evacuated, filled, and then pressurized to increase the acoustic velocity. This is exactly how these tests were conducted. Referring to Fig. C-1, the air space over the liquid in the fill tank was evacuated, allowing dissolved gases to evolve. The canister was also evacuated. Liquid was then pulled into the canister until it flowed out the top. The top and bottom valves were closed producing a solid, deareated, fluid-shell system. The system was then pressurized through an accumulator to a pressure of several atmospheres. The acoustic velocity in water was calculated from bulk modulus measurements as 600 m/sec using this filling technique. It was noted that there was little difference between the measured natural frequencies with the accumulator valved in or out even though, with the accumulator valve open, the acoustic velocity was substantially less. 
The disadvantage of the hamonic excitation technique is that resonances other than the shell resonances are excited. These include the fluid column resonances and resonances in the fluid annular region. These resonances tend to be more pronounced on the pressure transducer signals and by noting phase relationships can be separated from the shell resonances.

Damping Ratios

Damping ratios were measured using the half-power method [C-3]. Using this method the damping is found by measuring the half-power bandwidth of the modal response from the strip chart frequency records and using the relationship

$$
\frac{\lambda}{C_{c}}=\frac{\Delta f}{2 f}
$$

where

$$
\begin{aligned}
\lambda & =\text { damping coefficient } \\
C_{c} & =\text { critical damping coefficient } \equiv 2 \mu_{\text {eff }} \Omega \\
\Delta f & =\text { bandwidth at the half-power points } \\
f_{c} & =\text { center frequency of the modal response }
\end{aligned}
$$

The half-power bandwidth is the difference in frequency between the half amplitude point before and after the resonance is reached.

\section{Radial Displacements}

Radial displacements were computed on TDAC by integrating the radial acceleration twice. Any dc component of the signal was removed after each integration by fitting a linear curve through the data and then subtracting out the linear curve. If necessary the acceleration signal could be mathematically filtered to a selected frequency bandwidth to eliminate 
extraneous noise. This was found unnecessary in those cases where the shell's response was a fairly pure sine wave. The integrated displacement

matched within 10\% that which would be calculated by assuming a harmonic excitation.

where

$$
w(t)=-\frac{\ddot{H}(t)}{\Omega^{2} \sin \Omega t}
$$

$$
\begin{aligned}
W(t) & =\text { radial displacement } \\
W(t) & =\text { radial acceleration } \\
\Omega & =\text { circular frequency }
\end{aligned}
$$

\section{EXPERIMENTAL RESULTS}

Natural frequencies were determined in air and under water using the impact technique and under water and oil using the harmonic technique. Properties of the oil used are given in Table C-3.

\section{Table C-3 - Transformer 0il Properties}

$$
\begin{aligned}
& \text { density }-880 \mathrm{~kg} / \mathrm{m}^{3}\left(0.032 \mathrm{lb} / \mathrm{in}^{3}\right) \\
& \text { kinematic viscosfty at } 100^{\circ} \mathrm{F}-12 \mathrm{~mm}^{2} / \mathrm{sec}(12 \text { centistokes) } \\
& \text { dielectric strength, } 0.1 \text { in. gap at } 25^{\circ} \mathrm{C}-30 \mathrm{kV} \\
& \text { specification - VV - I - } 530 \mathrm{Class} 2
\end{aligned}
$$

Results of the frequency measurements are given in Tables $C-4 A$ and $C-4 b$ for the fixed-free and fixed-fixed end conditions. As can be seen, the natural frequencies measured by the two different techniques closely agree. Note that two natural frequencies are reported for the $(3,2)$ mode ill air. This is probably due to the method of boundary constraint used as has been reported by others [C-4]. 
Table C-4a

Natural Frequencies and Modes for Fixed-Free End Conditions

\begin{tabular}{|c|c|c|c|c|c|}
\hline \multirow{2}{*}{$n$} & \multirow{2}{*}{$\mathrm{m}$} & Air & \multicolumn{2}{|c|}{ Water } & $0 i 1$ \\
\hline & & Impact & Impact & Harmonic & Harmonic \\
\hline 2 & $\begin{array}{l}1 / 2 \\
3 / 2\end{array}$ & $\begin{array}{l}200 \mathrm{~Hz} \\
740\end{array}$ & $\underset{315}{75} \mathrm{~Hz}$ & & \\
\hline 3 & $\begin{array}{l}1 / 2 \\
3 / 2 \\
5 / 2\end{array}$ & $\begin{array}{r}440 \\
620 \\
1150\end{array}$ & $\begin{array}{l}195 \\
300 \\
625\end{array}$ & $195 \mathrm{~Hz}$ & $205 \mathrm{~Hz}$ \\
\hline 4 & $\begin{array}{l}1 / 2 \\
3 / 2 \\
5 / 2\end{array}$ & $\begin{array}{l}820 \\
890\end{array}$ & $\begin{array}{l}420 \\
485 \\
675\end{array}$ & $\begin{array}{l}420 \\
470 \\
610\end{array}$ & 435 \\
\hline 5 & $\begin{array}{l}1 / 2 \\
3 / 2 \\
5 / 2\end{array}$ & $\begin{array}{l}1325 \\
1375\end{array}$ & $\begin{array}{l}750 \\
815 \\
945\end{array}$ & $\begin{array}{l}755 \\
795 \\
890\end{array}$ & 780 \\
\hline
\end{tabular}

Table $c-4 b$

Natural Frequencies and Modes for Fixed-Fixed End Conditions

\begin{tabular}{|c|c|c|c|c|c|}
\hline \multirow{2}{*}{$n$} & \multirow[b]{2}{*}{$\mathbf{m}$} & \multirow{2}{*}{ Air } & \multicolumn{2}{|c|}{ Water } & \multirow{2}{*}{$\frac{0 i 1}{\text { Harmonic }}$} \\
\hline & & & Impact & Harmonic & \\
\hline 2 & 1 & $530 \mathrm{~Hz}$ & $200 \mathrm{~Hz}$ & & \\
\hline 3 & $\begin{array}{l}1 \\
2\end{array}$ & $\begin{array}{l}550 \\
925,1005\end{array}$ & $\begin{array}{l}250 \\
475\end{array}$ & $240 \mathrm{~Hz}$ & $242 \mathrm{~Hz}$ \\
\hline 4 & $\begin{array}{l}1 \\
2 \\
3\end{array}$ & $\begin{array}{r}860 \\
1050 \\
1440\end{array}$ & $\begin{array}{l}440 \\
600 \\
860\end{array}$ & $\begin{array}{l}435 \\
555\end{array}$ & 451 \\
\hline 5 & $\begin{array}{l}l \\
2\end{array}$ & $\begin{array}{l}1350 \\
1460\end{array}$ & $\begin{array}{l}775 \\
880\end{array}$ & $\begin{array}{l}765 \\
835\end{array}$ & 795 \\
\hline
\end{tabular}


The method of determining naturai frequencies and modes using the impact technique is illustrated in Figs. C-4 and C-5. Fig. C-4 is a flot of the amplitude of the imaginary part of the transfer iunction, $|\operatorname{Im}(j \omega)|$, for two different end conditions with the shell under water. These particular plots resulted from impacts at location 1 (see F1g. C-3). The peaks on the frequency plots are possible natural frequencies. By viewing a three dimensional plot of $|\operatorname{Im}(j \omega)|$ for each point struck at each frequency peak the true natural frequencies are verified when a clear modal profile springs into focus. Pictures of some of these modes are siown in Figs. C-5a,.., h. By counting the number of times the maximum displacenent trace (solid line) crosses the shell autline (dotted ine) the mode number can be identified.

A harmonic frequency sweep technique was used to verify the natural frequencies in water and also to measure the natural frequencies in 011. Qil, with $90 \%$ the density of water, provides another check on the validity of the assumptions used in SHELVIB, the code developed for this research. Transfomer oil was used because of its insulating properties.

Typical pressure and acceleration records with the shell immersed in water are shown in Figs, $\mathrm{C}-6$ and $\mathrm{C}-7$. The exciter head peak acceleration was held constant at $9.8 \mathrm{~m} / \mathrm{s}^{2}$ for these runs. In Fig. $\mathrm{C}-6$ the responses, as measured on a strip chart recorder whlle the source frequency is varied, are shown for fixed-free and fixed-fixed end condltiuns. The pressure was introduced through one canister port or both ports simuitaneousiy for each zype end condition. Many resonances show up on these records and a careful look at the phase and amplitude relatjonships at each resonance and the phase changes between resonances was required to make positive 


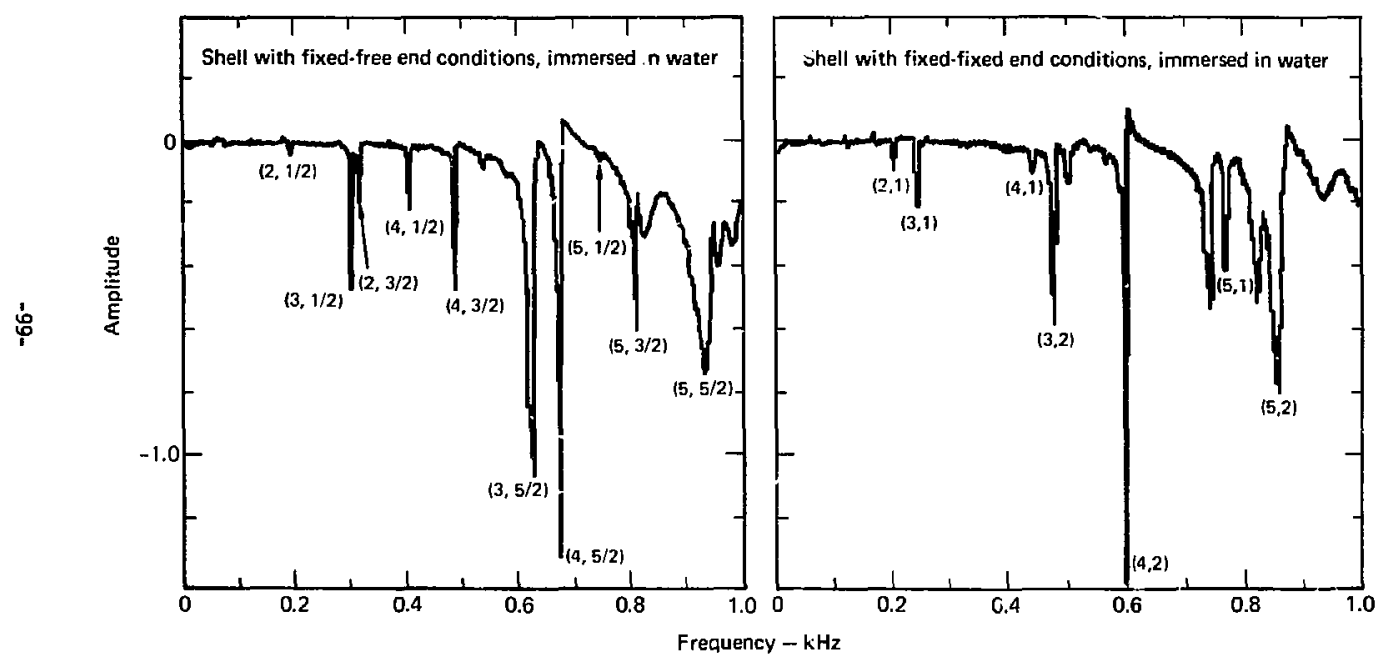

Fig C-4 Amplitude of the imaginary part of the transfer function. 


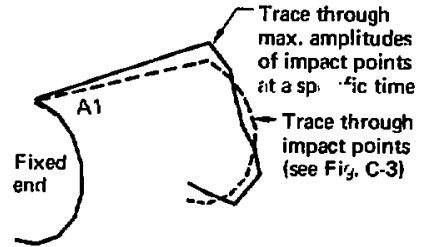

(a) Frequency $193 \mathrm{~Hz}$ mode $3,1 / 2$ fixed-free

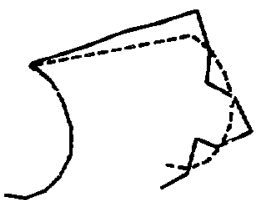

(b) Frequency $418 \mathrm{~Hz}$ mode $4,1 / 2$ fixed-free

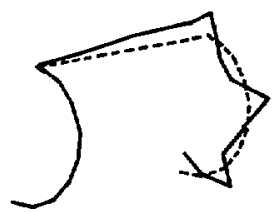

(c) Frequency $750 \mathrm{~Hz}$ mode $5,1 / 2$

: fixed-free

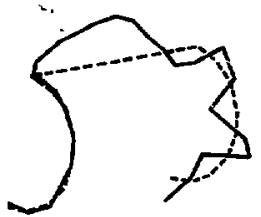

(d) Frequency $813 \mathrm{~Hz}$ mode $5,3 / 2$ fixed-free

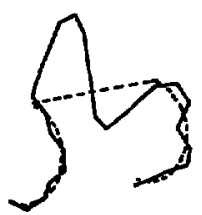

(e) Frequency $947 \mathrm{~Hz}$ mode $5,5 / 2$ fixed-free

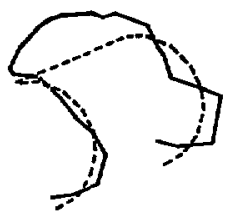

(f) Frequency $248 \mathrm{~Hz}$ mode 3,1 fixed-fixed

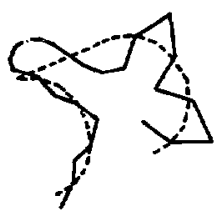

(g) Frequency $601 \mathrm{~Hz}$ $\bmod =4,2$

fixed-fixed

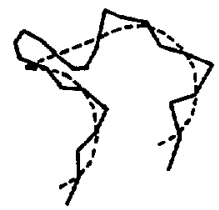

(h) Frequency $861 \mathrm{~Hz}$ mode 4,3 fixed-fixed

Fig. C-5 Shell modes when immersed in water determined from impact technique. Shell was hit at 26 locations along dotted !ine and response recorded by accelerometer A1. From these measurements the relative amplitude at each impact point for each mode was determined. 


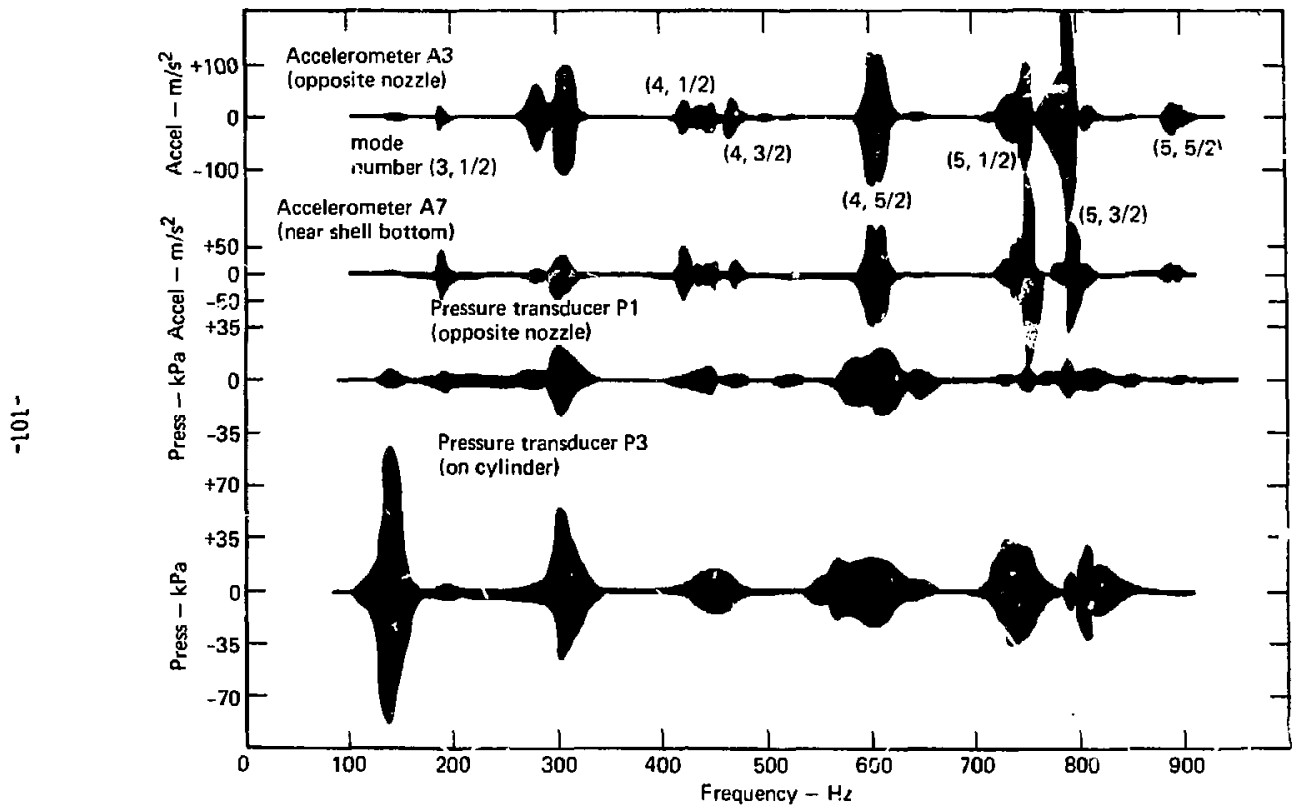

Fig. C-6a Shell response vs. exciration frequency; one nozzle open, shell with fixed.free end condtitons, immersed in woter. 


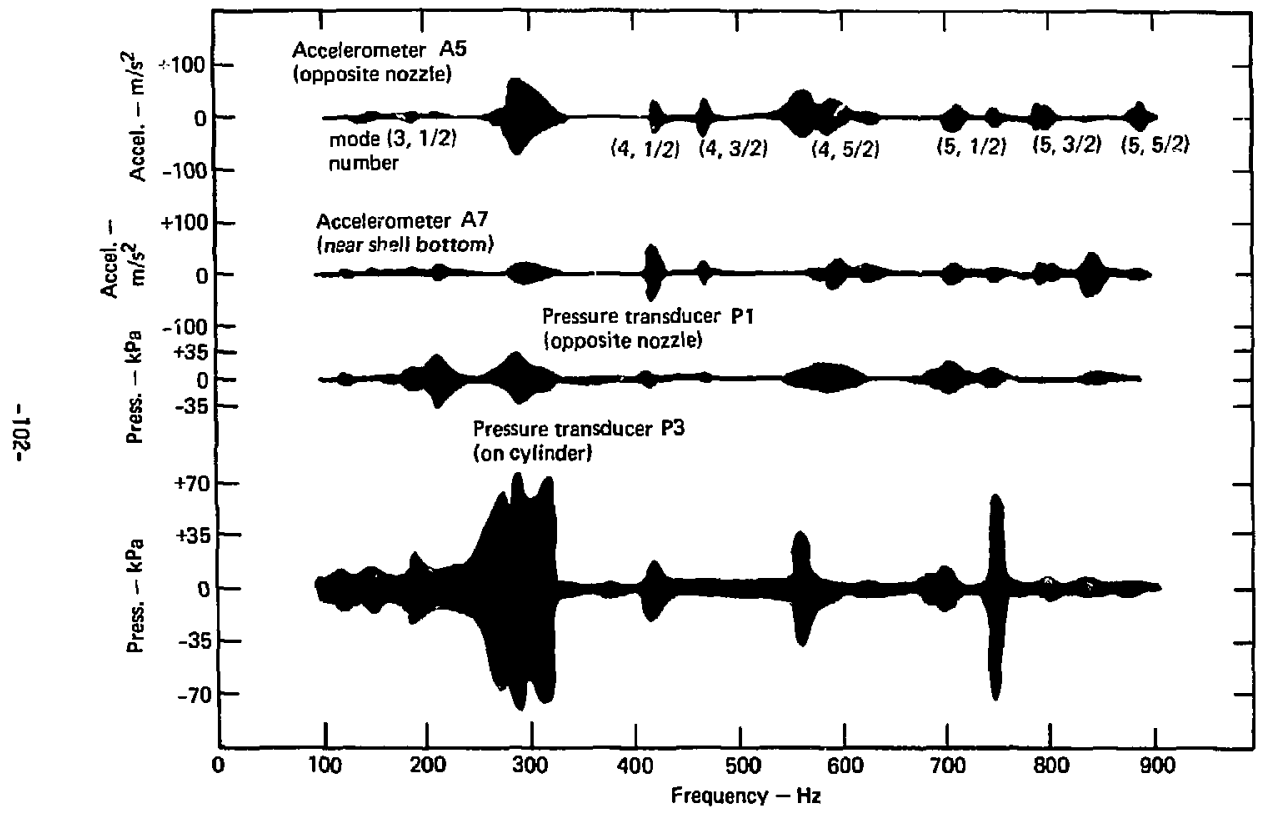

Fig. C-6b Shell response vs. excitation frequency: two nozzles open, shell with fixed-free end conditions, immersed in water. 


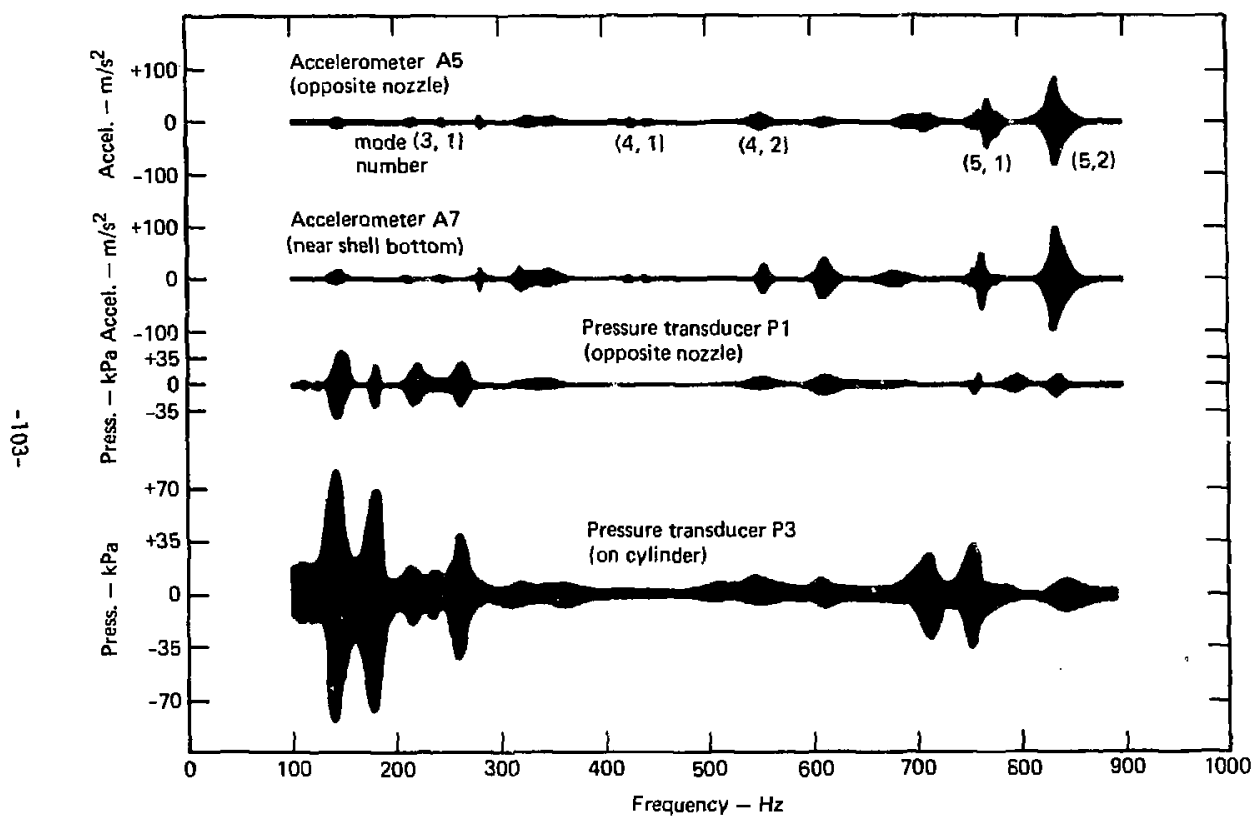

Fig. C-6c Shell response vs. excrtation frequency; one nozzle open, shell with fixed-fixed end conditions, immersed in water. 


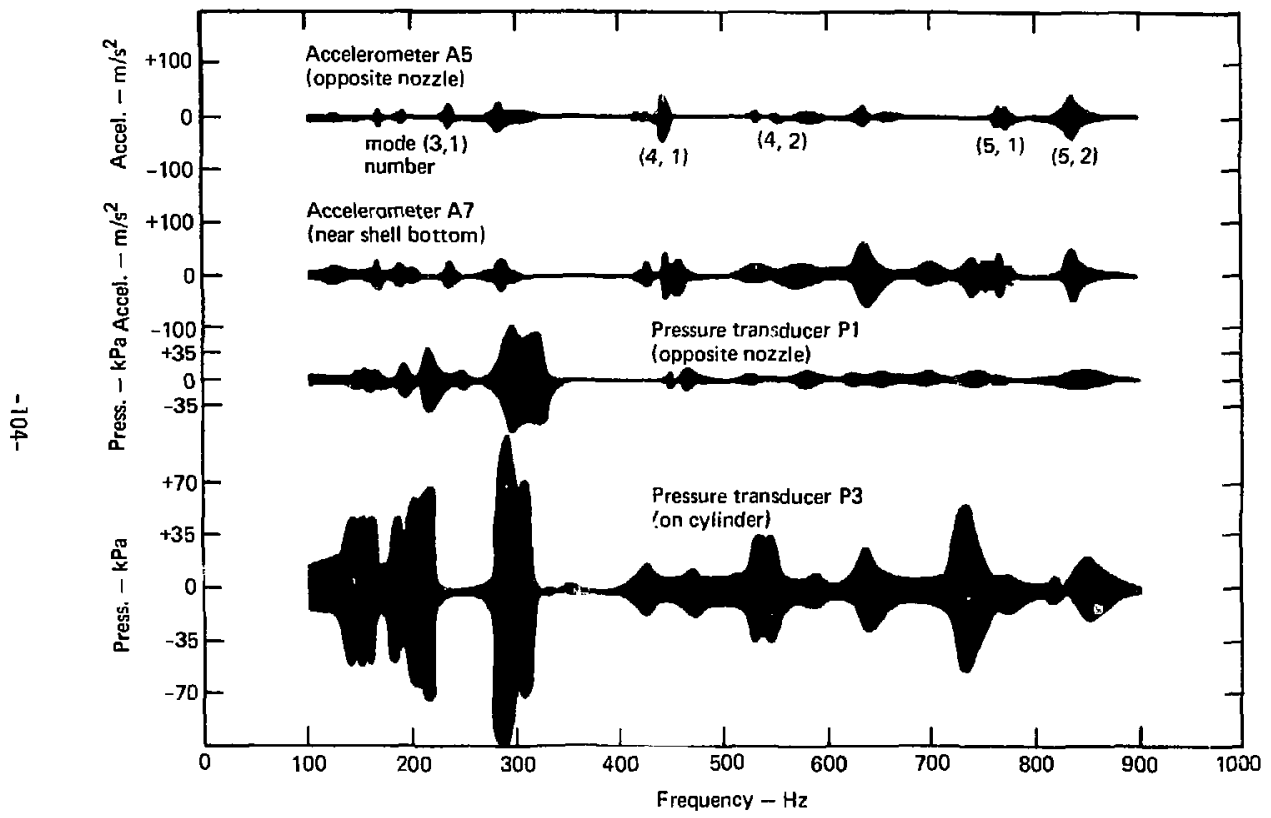

Fig. C.6d Shell response vs. excitation frequency; two nozzles open, shell with fixed-fixed end coriditions, immersed in water. 


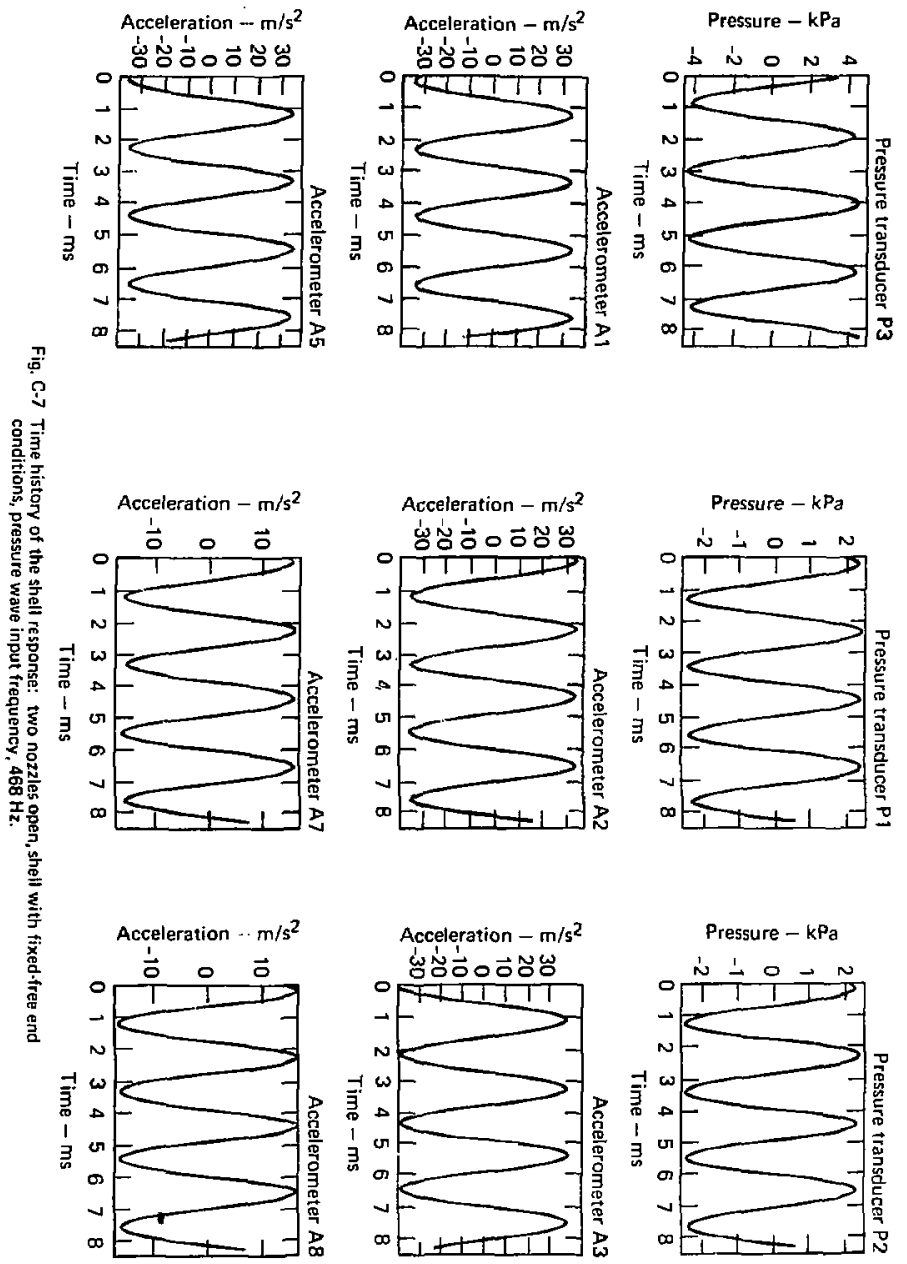


identification of those which correspond to shell natural frequencies. Even so, some resonances could not be resolved, i.e. the $(2,3 / 2)$, $(3,3 / 2)$ and a vater column resonance all occur riear $300 \mathrm{~Hz}$ (see Figs. $\mathrm{C}-6 \mathrm{a}, \mathrm{b})$, but could not be resolved. In general, if two modal frequencies where closer than $15 \mathrm{~Hz}$ they could not be resolved. At frequencies below $150 \mathrm{~Hz}$ the feedback from the fluid system to the exciter was such as to distort the harmonic wave form making data unusable below that point.

Time history records of the piston pressure, shell surfa.i. pressure, and the shell response at various points on the shell at the $(d, 3 / 2$. resonance are shown in Figs. C-7. The response data was cantured at an iristant of time, digitized, recorded, and then displayed, as shown, using TDAC. Comparison of the amplitude and phase of the accelerometer signals helped to identify the mode excited. Far instance, comparison of the signal from accelerometers $A 3$ and $A 7$ show that they are 180 degrees out of phase with the $A 7$ signal half the amplitude of the A3 signal. Since $A 3$ is near an anti-node of the $(4,3 / 2)$ mode and $A 7$ below the node this verified that the $3 / 2$ mode is the axial mode excited. In a similar manner other modes were identified.

Modal damping ratios in the fluid are shuwn in Table $\mathrm{C}-5$. These are close to the values to be expected from structural damping aione. For these particular modes, in this geometry, damping caused by the fluid appears minimal.

Figures $C-B a, \ldots$, d illustrate how the radial displacement was computed by numerical integration of the radial acceleration record shown in Fig. C-8a. Even though the acceleration was distorted hy an 
odd-harmonic of the input frequency (see the fourier transform of the accelerometer signal in Fig. (-8b) the displacement peak computes to the value that would be calculated assuming simple hamonic motion (c-2). Fig. C-8c shows the radial velocity computed by integration of the acceleration signal. Fig. C-8d shows the radial displacement computed by integration of the radial velocity. Accelerometer $A 1$ is located mid-way between the inlet ports and the excitation was at the resonant frequency of the $(3,1 / 2)$ mode in water.

$$
\begin{aligned}
& \text { Table } \mathrm{C}-5 \text { - Damping Ratios, } \frac{\lambda}{\mathrm{C}_{\mathrm{C}}} \\
& C_{c}=2 M_{e f f}
\end{aligned}
$$

\begin{tabular}{|c|c|c|c|c|c|c|c|}
\hline \multicolumn{4}{|c|}{ Fixed-Free Shell End Conditions } & \multicolumn{4}{|c|}{ Fixed-Fixed Shell End Conditions } \\
\hline n & m & water & oil & $n$ & m & water & $0 i 1$ \\
\hline 3 & $1 / 2$ & $0.40 \%$ & $0.7 \%$ & 3 & 1 & $0.38 \%$ & $0.8 \%$ \\
\hline 4 & $\begin{array}{l}1 / 2 \\
3 / 2 \\
5 / 2\end{array}$ & $\begin{array}{l}0.24 \\
0.21 \\
0.30\end{array}$ & 0.4 & 4 & $\begin{array}{l}1 \\
2\end{array}$ & $\begin{array}{l}0.23 \\
0.29\end{array}$ & 0.7 \\
\hline 5 & $\begin{array}{l}1 / 2 \\
3 / 2 \\
5 / 2\end{array}$ & $\begin{array}{l}0.12 \\
0.12 \\
0.15\end{array}$ & 0.2 & 5 & $\begin{array}{l}1 \\
2\end{array}$ & $\begin{array}{l}0.14 \\
0.16\end{array}$ & 0.2 \\
\hline
\end{tabular}



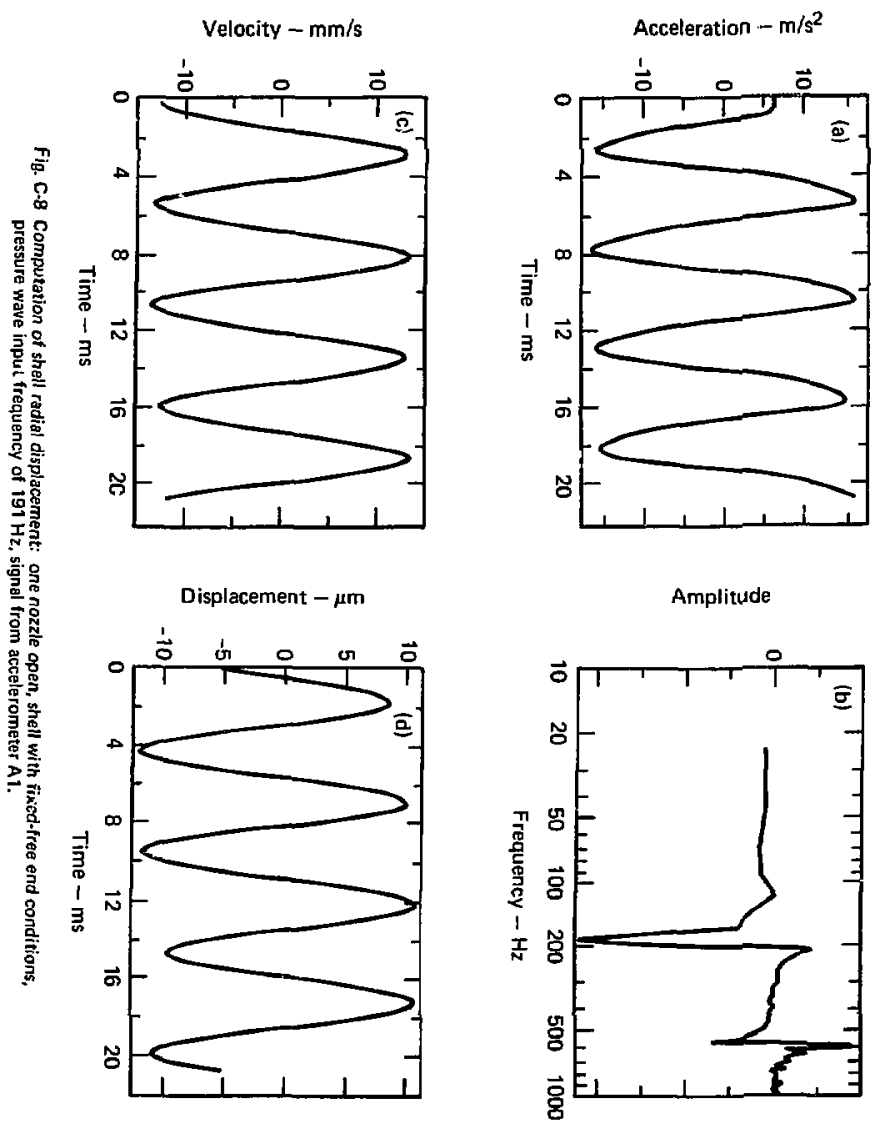
C-1. Fisher, D. K., Posehn, M. R., Sindelar, F. L., and Bell, H. H., "A Computer-Based Transportahle Data Acquisition and Concrol System," Lawrence Livermore Laboratory Report UCRL-78339, (Sept. 1976).

C-2. E'dington, R. B.s "In restigation of Supersonic Shock Phenomena in a Two-Phase (1 iquid-gas) Tunnel", NASA Tech. Rept. 32-1096, Jet Propulsion Lab., Pasadena, Calif. (March 1967).

C-3. Thomson, W. T., Theory of Vibration with Applications, PrenticeHall Inc., Englewood C1iffs, New Jersey, (1972).

C-4. Assedo, R., Epstein, A., Gilbert, R., and Livolant, M., "Synthes is of Vibration Studies on a Three Loop PWR Internais Model," Trans. Fourth Inter. Conf. on Structural Mechanics in Reactor Techñology, San Francisco, (1977), Paper F5/3. 
APPENDIX D - THE COMPUTER CODE SHELVIB

Included in this appendix is a listing of the computer code, SHELVIB, which was used to generate the results presented. Also included are the input and a portion of the output for a sample problem. SHELVIB is designed to run on a CDC 7600 computer and is coded in LRLTRAN [D-T], an extended version of FORTRAN IV. Note that the compiler used automatically sets all variabies and arrays to zero and can handle mixed mode arithmetic.

The computer code consists of a main program and seventeen subprogams. The main progran is used to call in the input, initialize variables, call the subroutines necessary to calculate the shell displacements during each time iteration, print out results, and plot the results. The principle subprograms are a printing routine (STRS), a plotting routine (FPLOTT), a poisson equation solver which calculates the fluid dynamic pressures (PWSCYL), and two matrix inversion routines (DEC/SOL, LINVIF). The remaining sub-programs help set up and manipulate the matrix shell equations. The nomenclature used is described in the code listing. Data is input using NAMELIST statements. Output can be requested for any or all space stations but is only plotted at the quarter points. The code calculates in the English system of units.

The sample problem is the calculation o $\overline{\mathrm{T}}$ the shell response to a $1 \mathrm{ps} i$, mid-point,step load with a $10 \mathrm{~ms}$ rise time. The shell is simply-supported at both ends and surrounded by water contained in a stiff-walled, coaxial, outer cylinder. The shell is empty. The calculation is for the 5 th circumferential mode $(\Omega / 2 \pi=50 \mathrm{hz})$. Oniy a partial output is shown. The olot is the time history of the non-dimensional radial displacement, $w$ at the midpoint. 


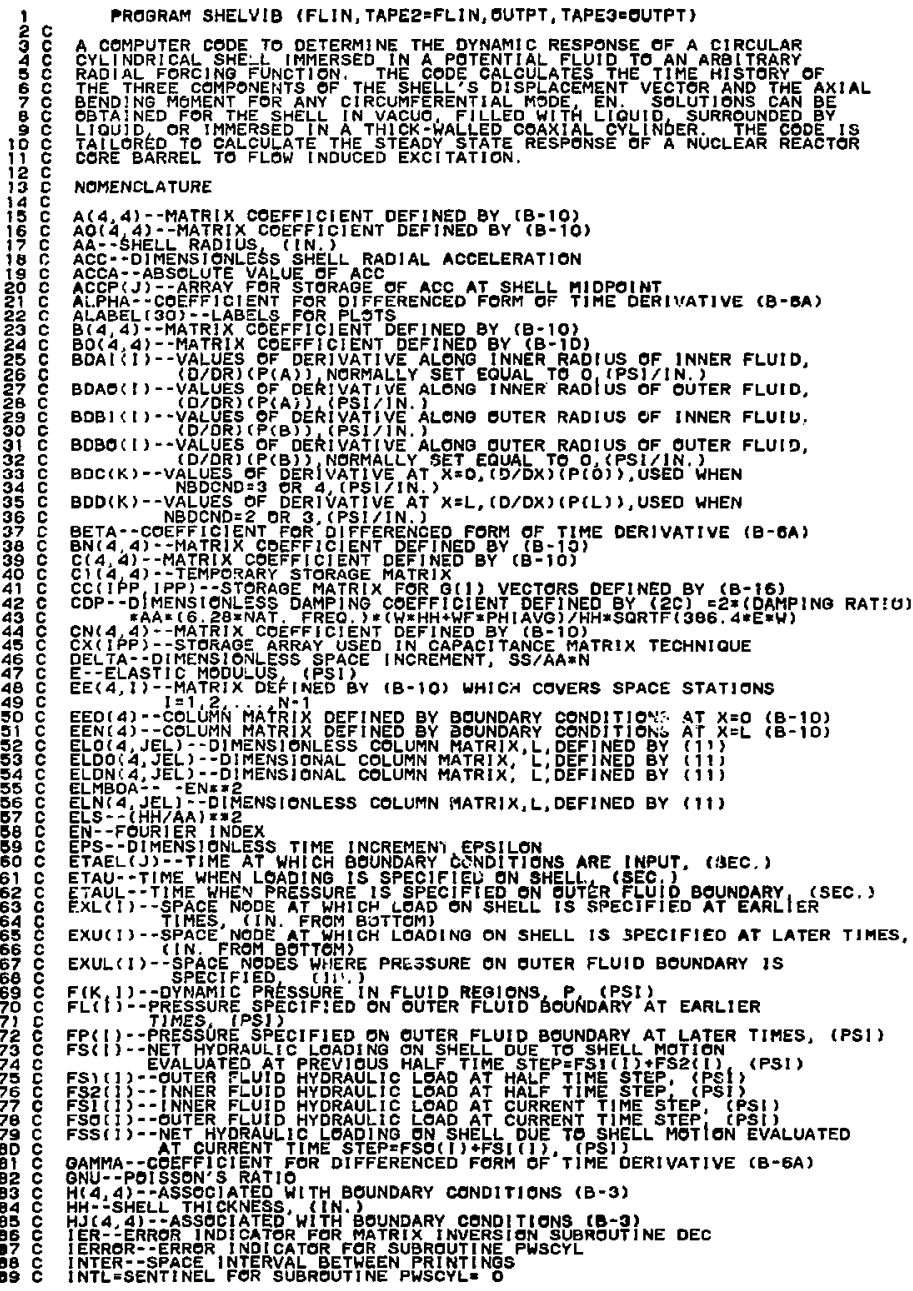


IP--PLOT POINT INDEX

IPIV(25) -STORAGE ARRAY USED IN CAPACI TANCE MATRIX TECHNIOUE IPRINT--TIME PRINTING CONTROL

IPP- NUMEER OF

IPRINOO-TIME INTERVALS BETHEEN PRINTINGS

IT--TIME STEP INDEX

JEL--NUMBEF OF TIMES THE BOUNOARY CONDITIONS ARE SPECIFIEO;

KP.-FIRST SPACE WODE AT HHICH EUTER BOUNDARY PRESSURE IS SPECIFIED

MBDCNDI--1NDICATES THE TYPE GF BEUNDARY CONDITIONS AT RIRIA AND R=RIB FOR INVER FLUID

$=2$ IF IHE SOLUTION IS SPECIFIED AT RERIA AND THE DERIVATIVE

= 3 IF THE SELUT TON WITH RESPECT TO R IS SPECIFIEO AT R=RI

MBDCNDQ - INDICATES THE TYPE OF BOLNDARY CONDITIONS AT R=ROA AND R=ROB

FOR OUTER FLUIO THE DIVIIVE OF THE SOLUTION WITH RESPECT TO R IS

- 4 SPETIFIEDAT R=RDA AND REROB

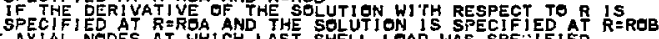

MOL:-NUMBER OE AXIAL NGDES AT WHICH LAST SHHEL LOAD WAS SPE IFIED

MRI:-NUMBER OF RAIAL NODES AT WHI CH NEXT SHELL LOAD NS

MRO- NUMBER EF RAOIAL INTERVALS IN OUTER FLUID REGION

N-NUMBER OF AXIAL SPACE INTERVALS

NIA N24 N3A-GUAATER HALF AND THREE-OUARTER AXIAL NODE NUMBERS

NBOLND I: -IND COATES THE TYPE OF BOUNDARY CONDITIONS AT $X=0$ AND $X=S S$ HOR INNER FLUIO

$=0$ IF THE SOLUT'ON IS PERIODIC IN $X$ I $\therefore P(O, K) \times P(N, K)$

- 1 IF THE SOLUTION IS SPECIFIED AT $k=0$ ANS $P$ TSK

- 2 IF THE SOLUTION IS SPECIFIEO AT X=O AND THE DERIVATILE

$=0$ IF THE DERIVATIVE OF THE SOLUTION WITH ECIFIECT TO XISS

- 4 IFECIFIED THE DERIVATIVE $X=0$ AND $x=5 S$ SPECIFIED AT X XO AND THE SOLUTION IS SOECIFIED AT XaSS NEDCNDO - INDICATES THE TYPE OF BOLNDARY CONDJ TIONS AT $X=D$ AND $X=S S$ FOR QUTER FLUID. NUMBER DESIGNATORS SAME AS NBDCNDI

NP-NUMBER OF POINTS TO BE PRINTED AND PLOTTED

DIIEGA- -FREOUNCY OF HARMCNIC SHEL LOAD WITH HALF AMPLITUPE OF OU, (HZ) OMEGAL - FREQUENEY OF HARMON1C PRESSURE PULSES ON

OUT-OSET EOUAL TO Y D IF OUTER FLUDE OFESENT

$P(4,4,1)$--ARRAY REPRESENTINO THE P MATRIX OF IB-11A) AT SPACE STATIONS

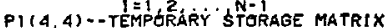

PERTKB- -UNUSED AROUHENT FOR SUBROUTINE PWSCYL=

PHI ( I) - CONNESTED MASS FACTOR FOR NODE I DEFTNED BY (1A), (IN.)

PHIAVO-PHI AVERAGED OVER ALL SPACE NODES IIN.

PHIAVGPIJ)-ARRAY FR STORAGE

PHIRUNS--RUNNING AVERAGE OF PHIAVG (IN.)

PIMAX ( 4O) -PPEAK SHELL LOAD FOR HARMGNIC LOADING (PS I)

RiA;-T-TEMPORARY STORADE COLUMN MATRLX

QU(I)--SPECIFIES SHELL LOAD AT THE PEINTS EXU(1), (PSI)

RIA--INNER FLUTD INAER RADIUS, TIN

RO--COEFFICIENT FOR DIFFERENCED FORM OF TIME DERIVATIVE IN (B-6A)

RDA--INNER RADIUS GF GUTER FLUID=AA IIN.)

ROC--BUTTOM OF SHELL. SET EOUAL TO ON.

SIA)- TEMPORARY STORAGE COLUHN MATRIX

SI OMA--TEFERENCE STRESS, (PSI)

ST 4,4$)$ TH

TEE- - CURRENT TIME (SEC)

TEPS - TIME INCREMENT ' (SEC ') WHEN TEE?TLIMIT (SEC.)

TM(3DO) - TIME COORDINATE ASSOCIATED WITH PLOTYED POINTS (SEC.)

TEMO (A) - -DIAGONAL ELEMENTS OF MATRIX OMEOA FROM BOUNDARY CONDITIONS

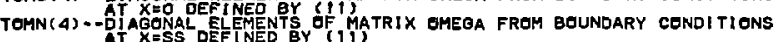

TT 1 (4 4 3OO) -TEMPORARY STORAGE MATRIX

H-SHELL WE J GHT DENS I TY (LBSTIN $=3$ 3)

HF-FLUID WEIOHT DENSITY (LES/IN,

WLE(NEMROJ--STORAOE ARRAÝ FER SUBROUTINE PUSCY

$X(4,1)--A R R A Y$ REPRESENTING THE X-PEAK OF (B-1IA) FOR SPACE STATIONS

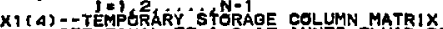

XIN--SETENUAL TO I.D IF INNER FLUTD PRESENT

XKAPPA - COEFFICIENT OF DIFFERENCED FORM OF TIME DERIVATIVE (B-6B)

XLAMBOA-COEFFICNT

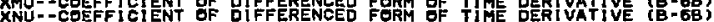

YMAX YMIN- MAXIMUM AND MINIMUM ORDINATES ON PLOTS

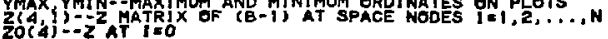




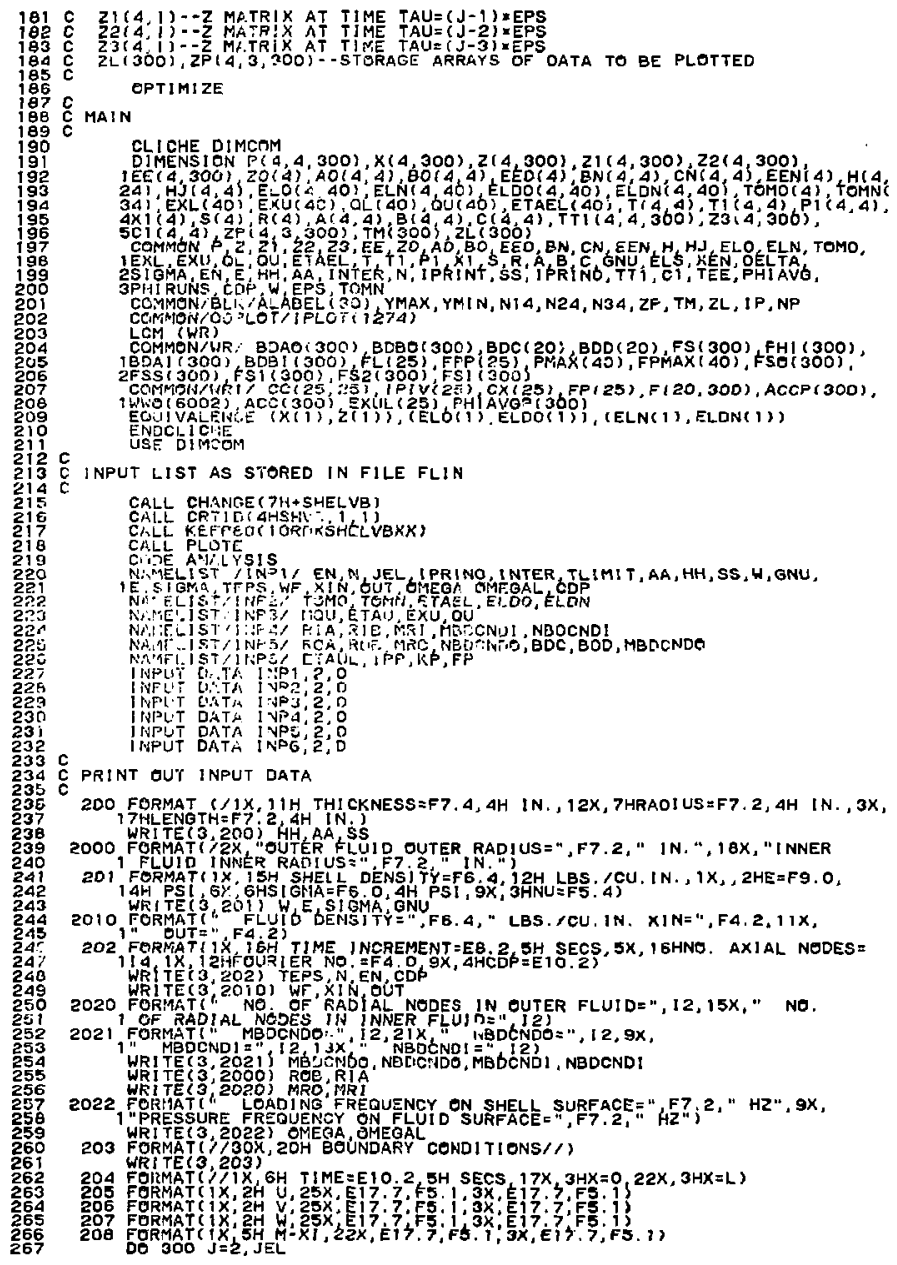




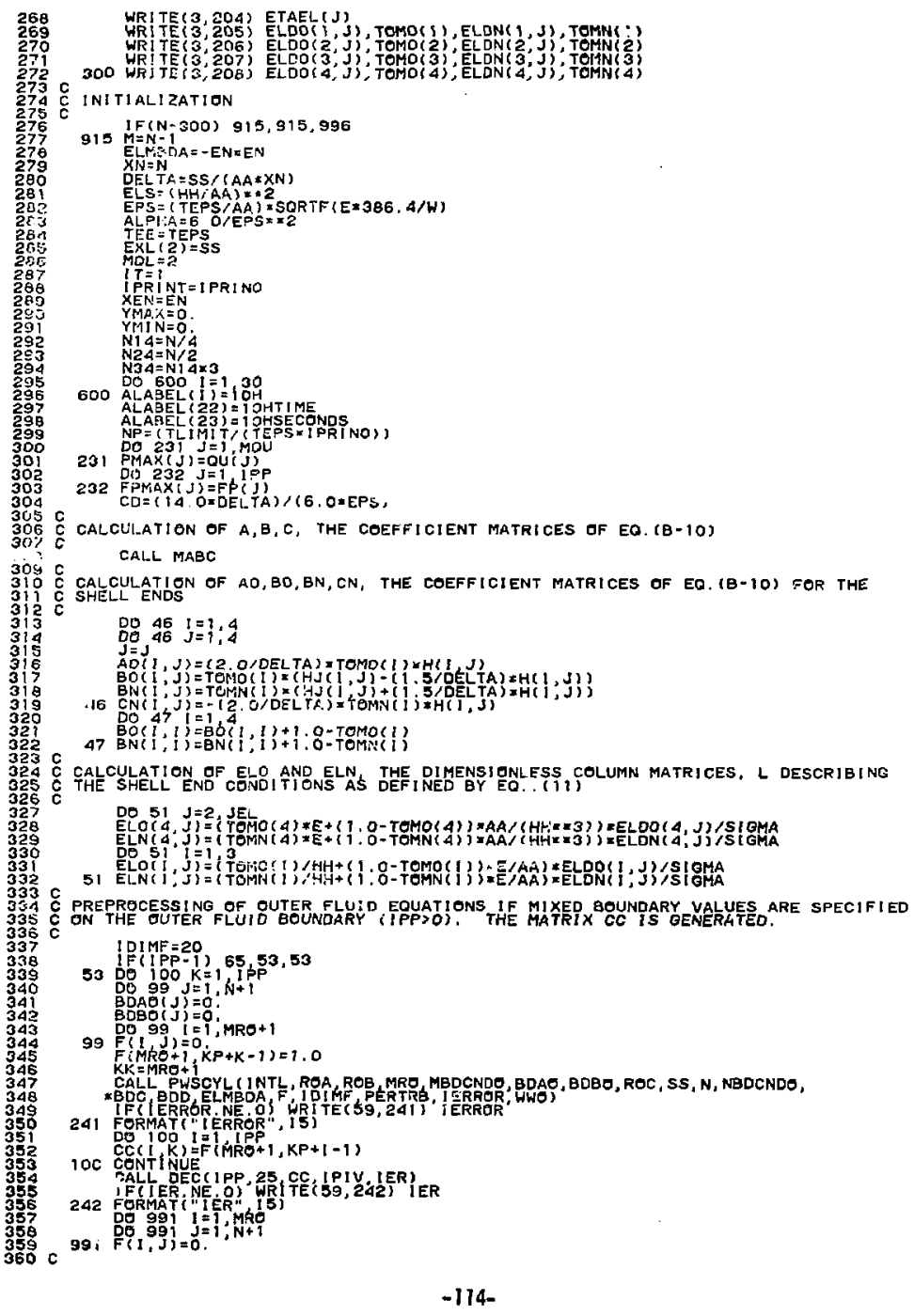




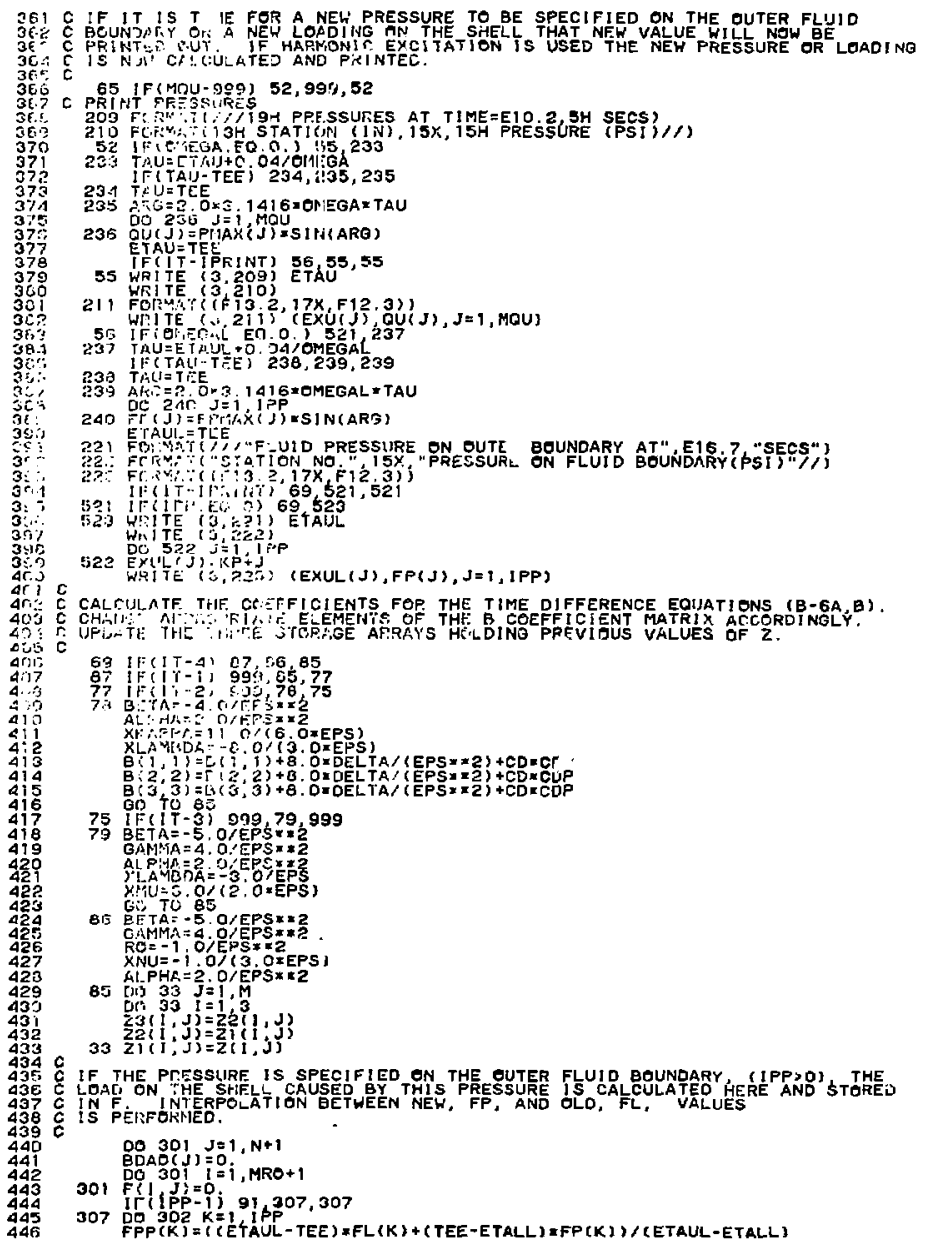




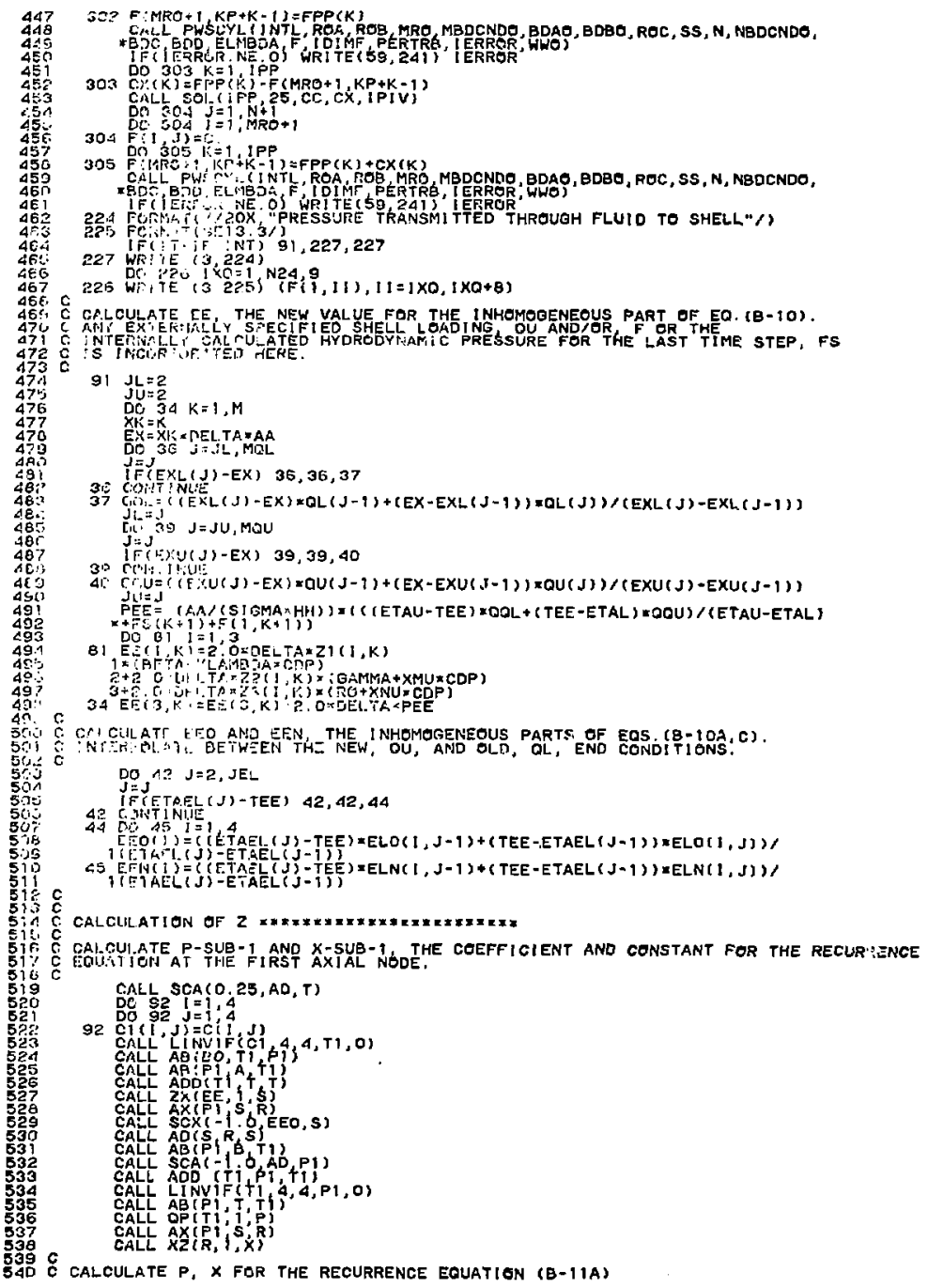




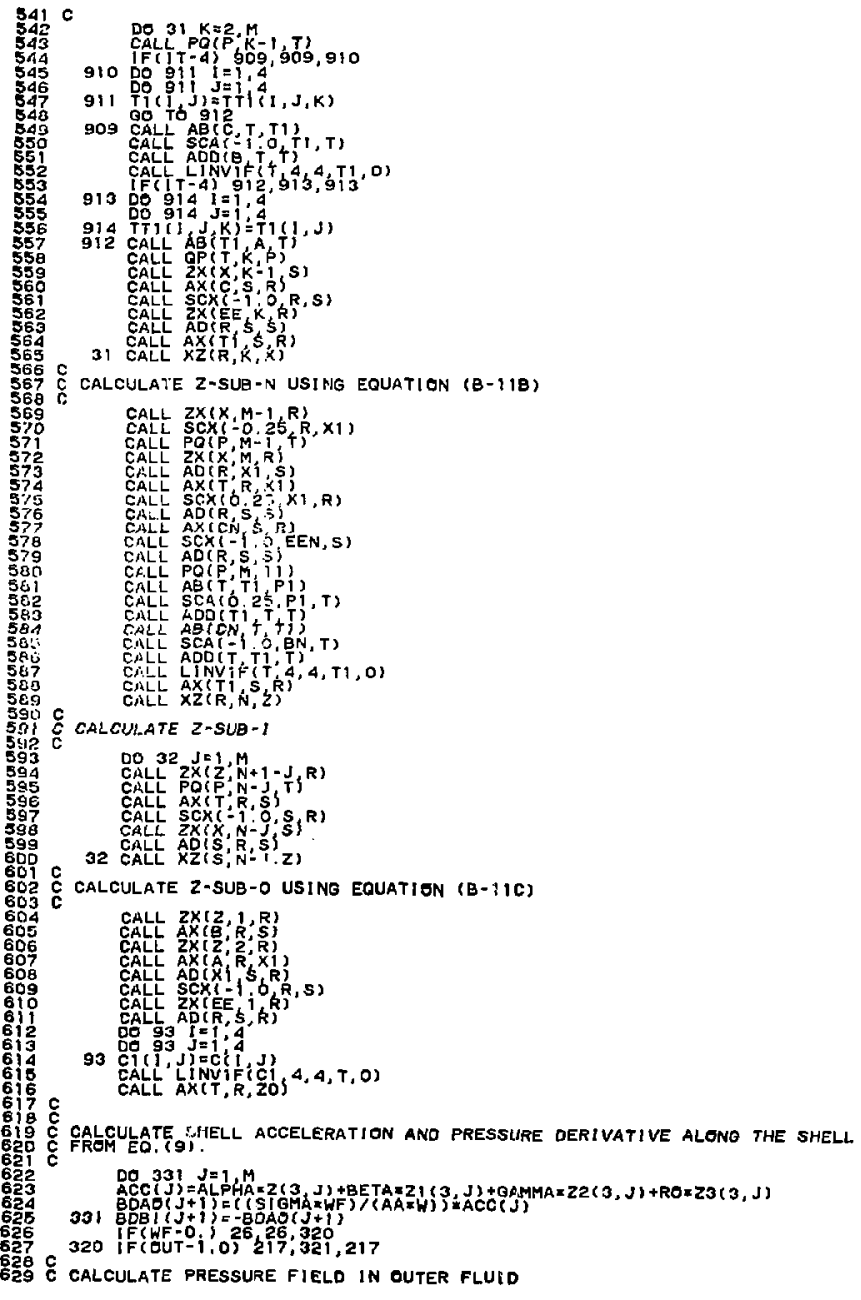




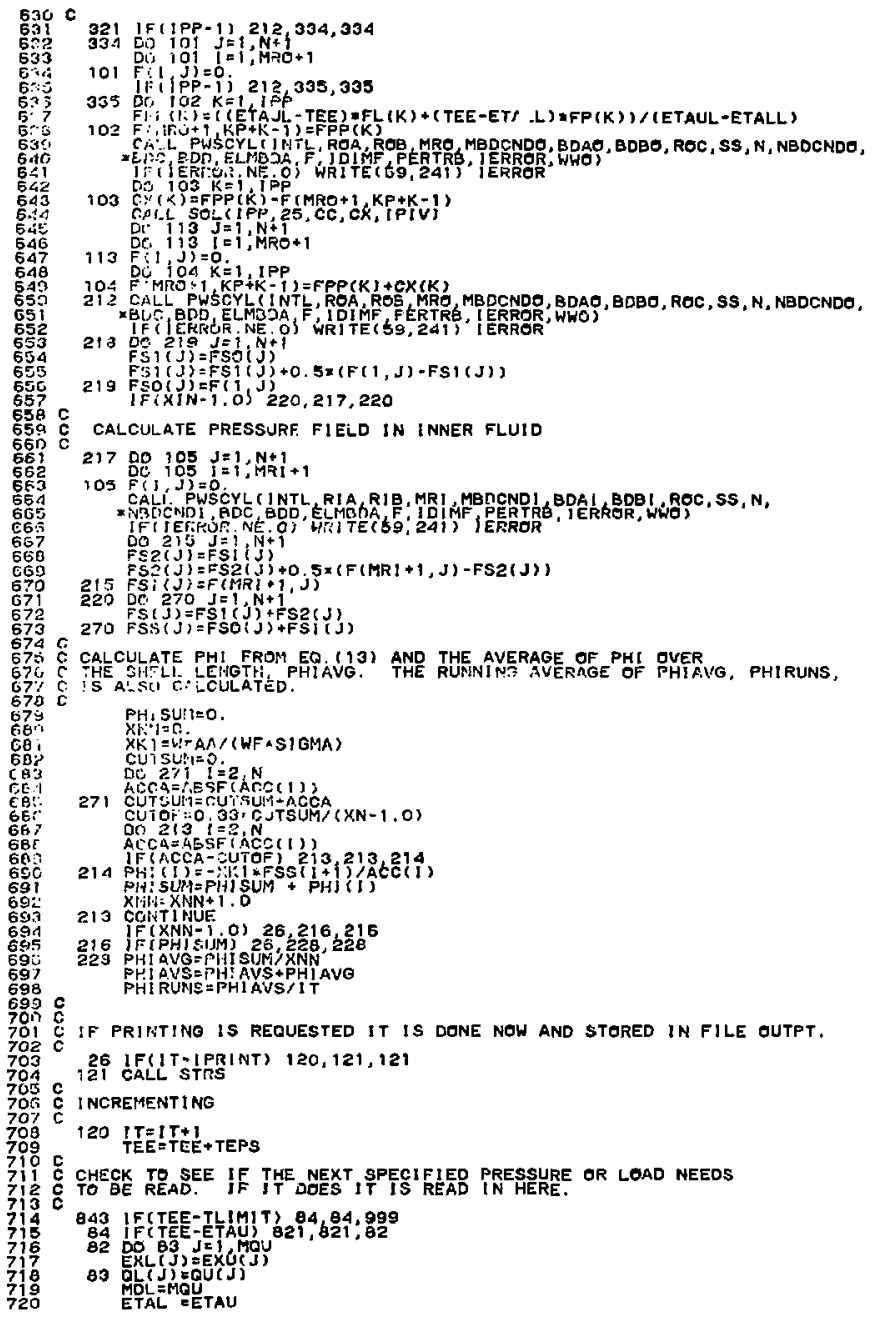




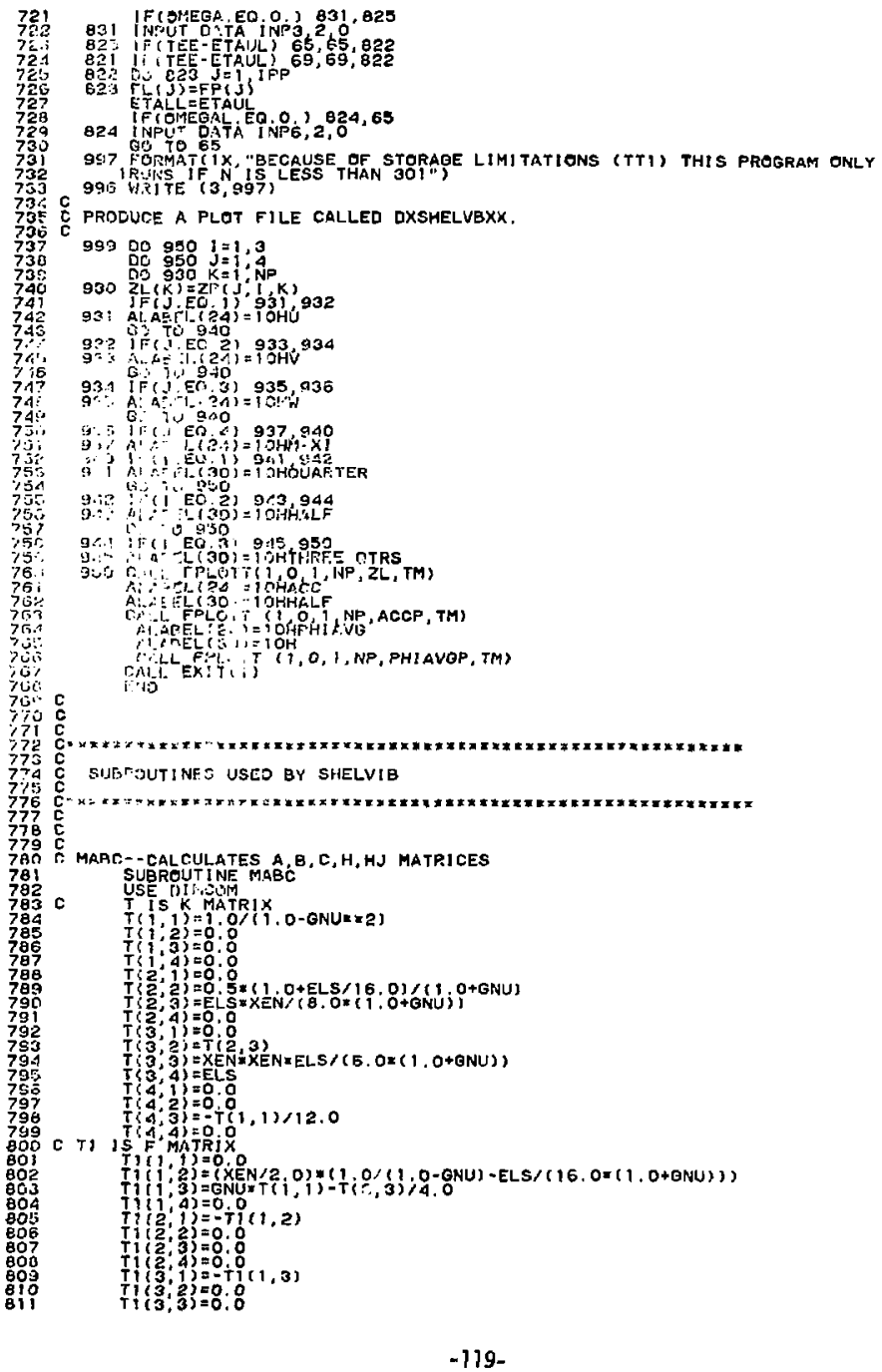




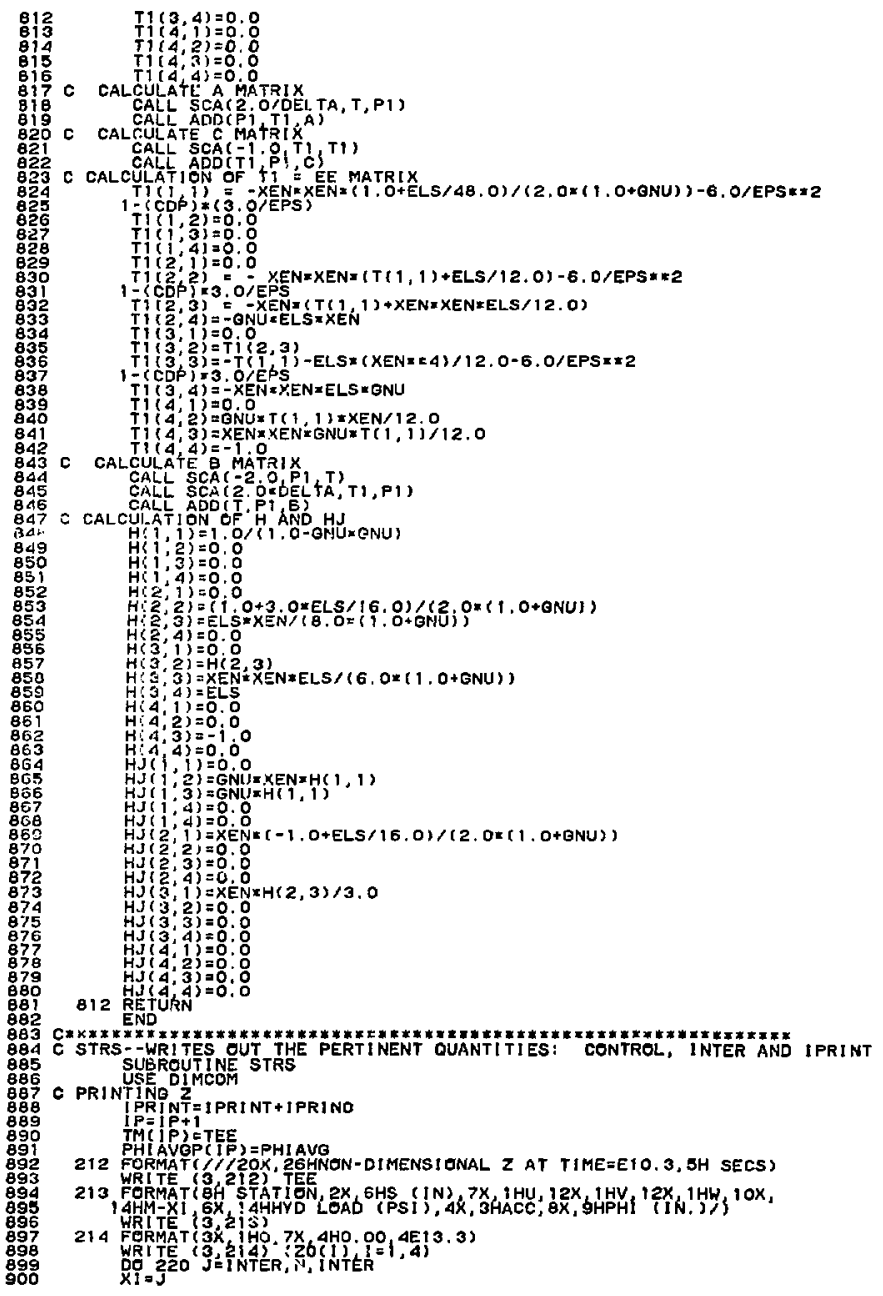




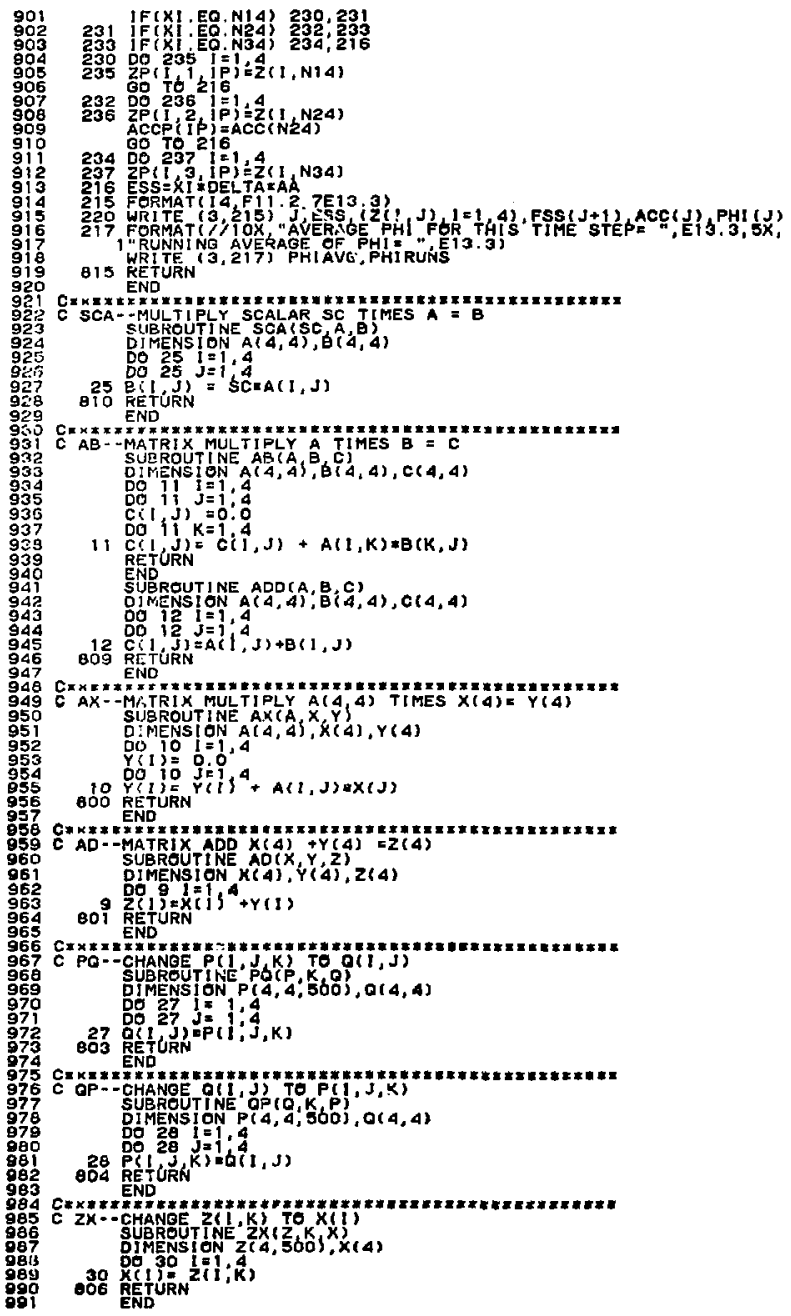




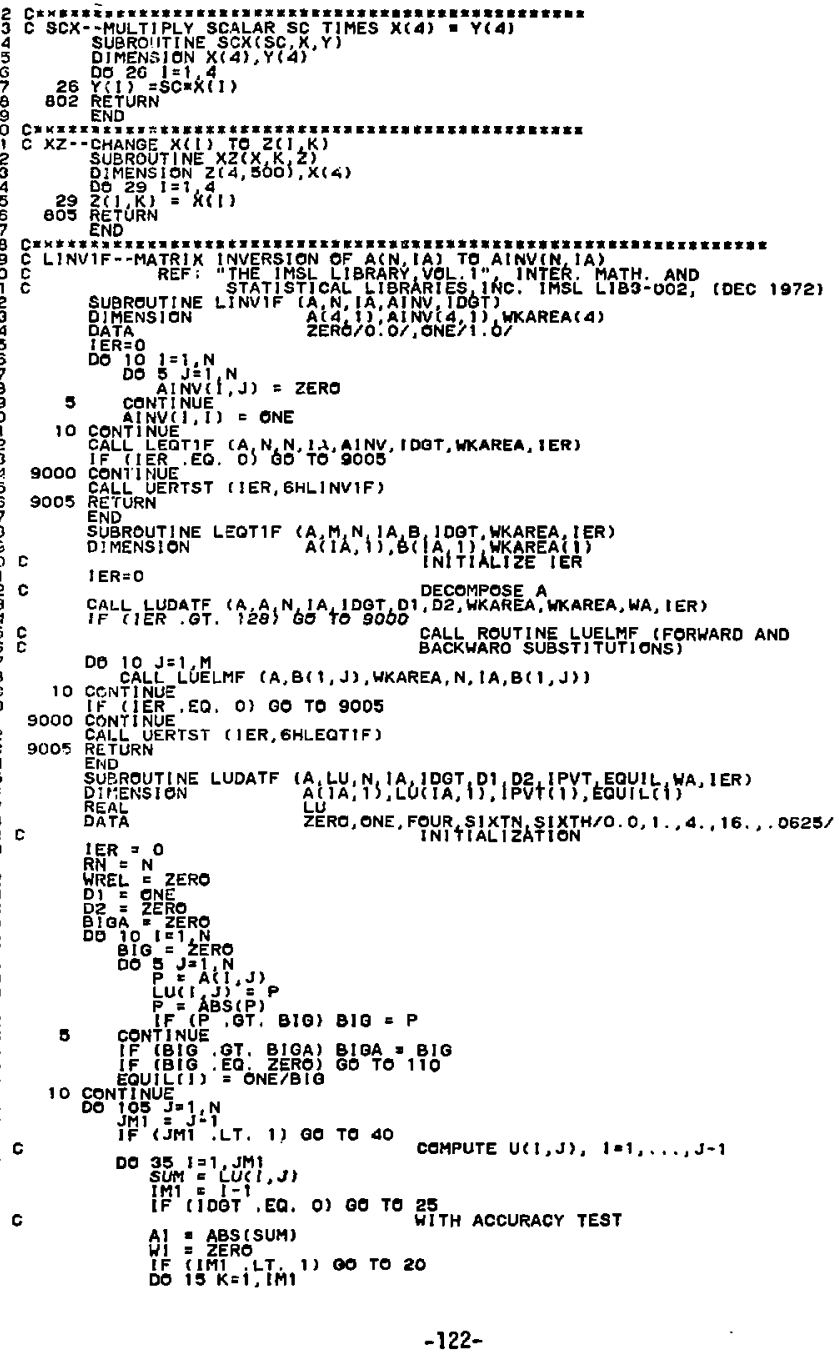




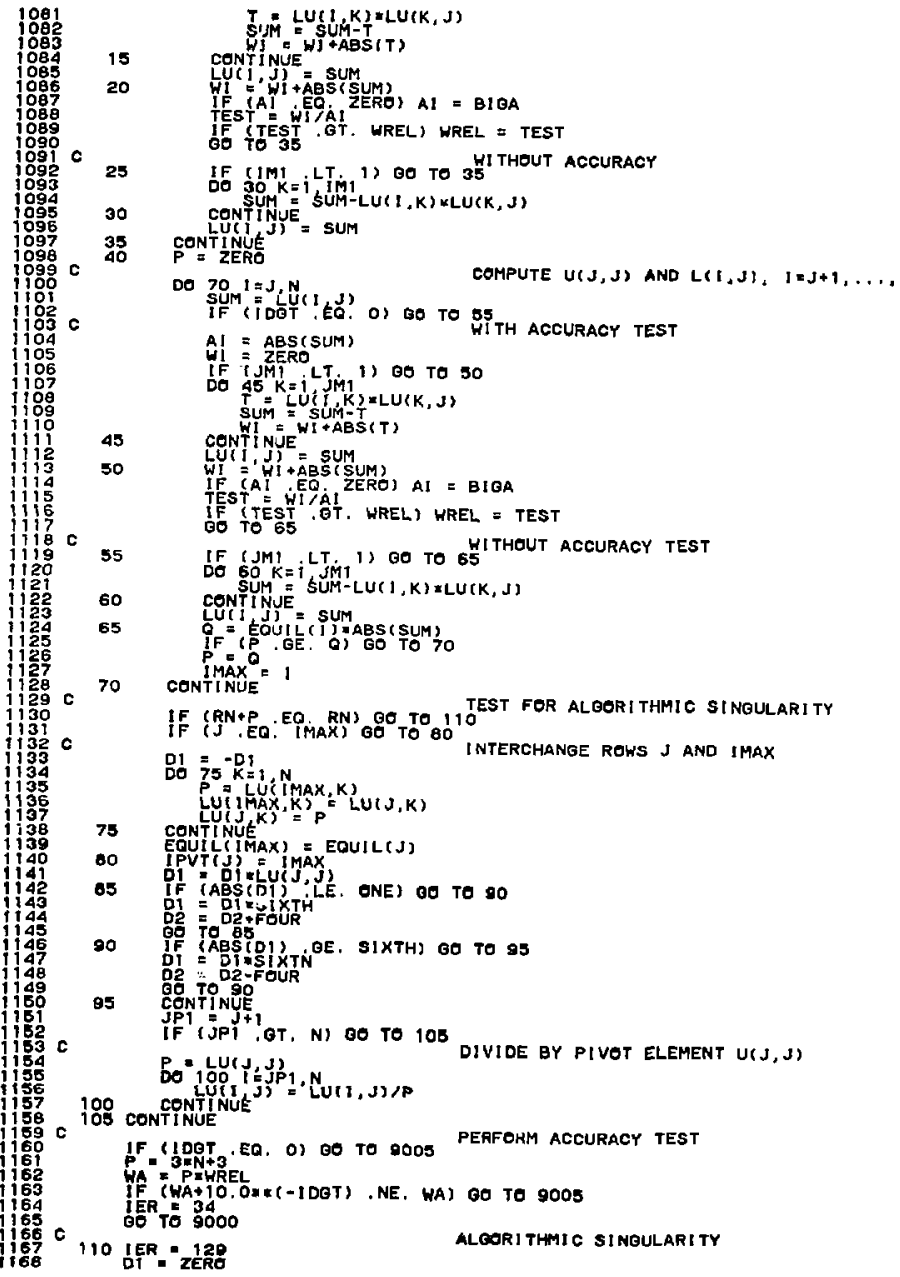




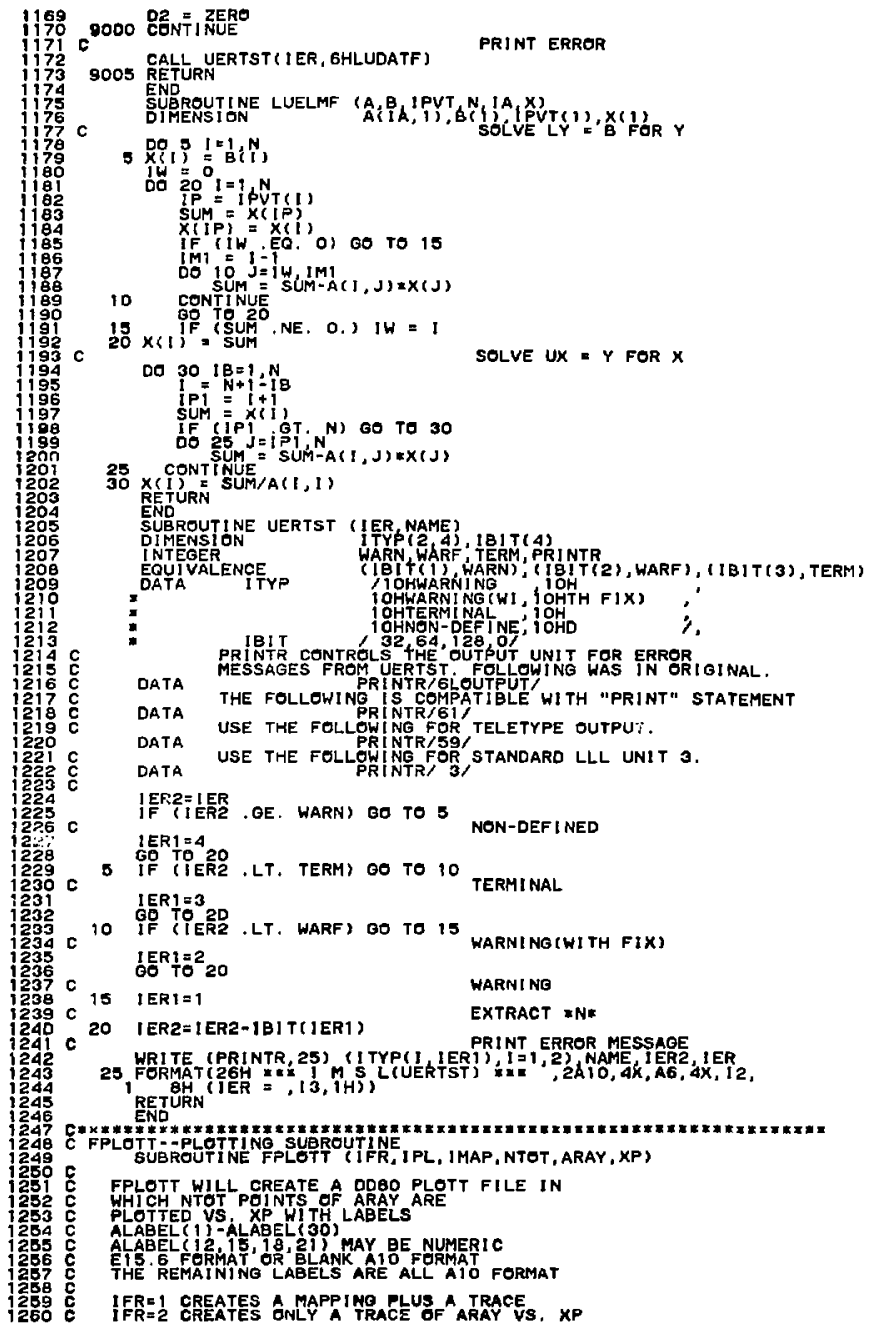




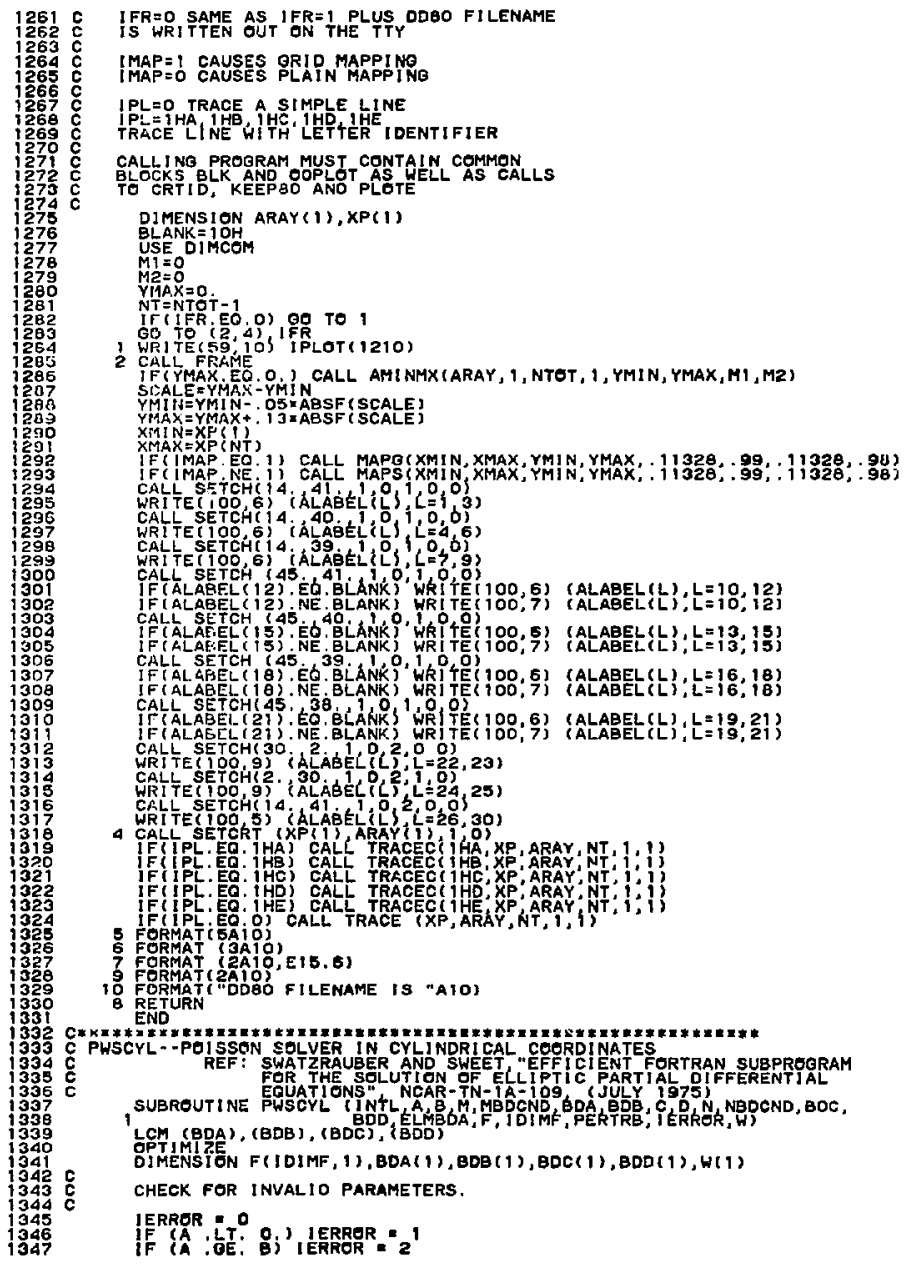




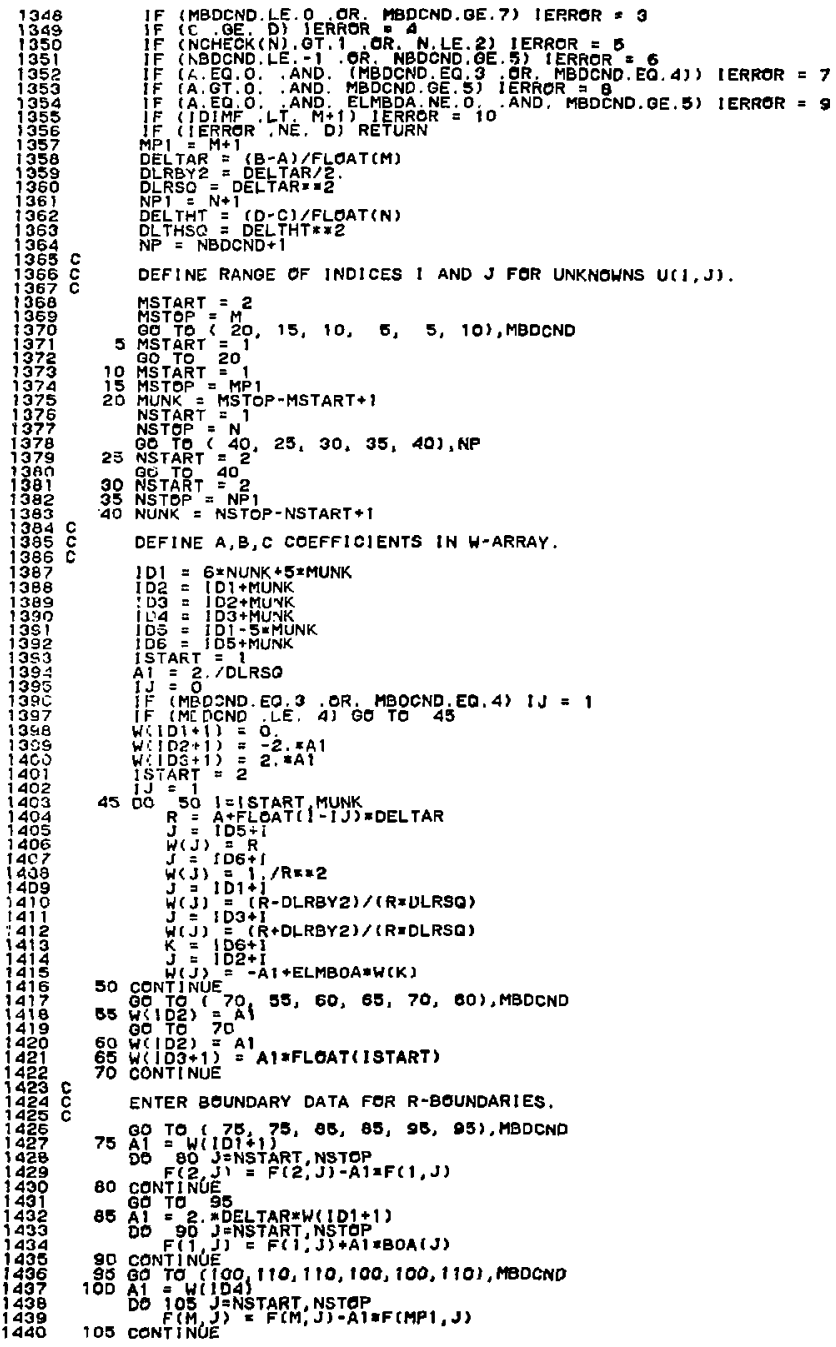




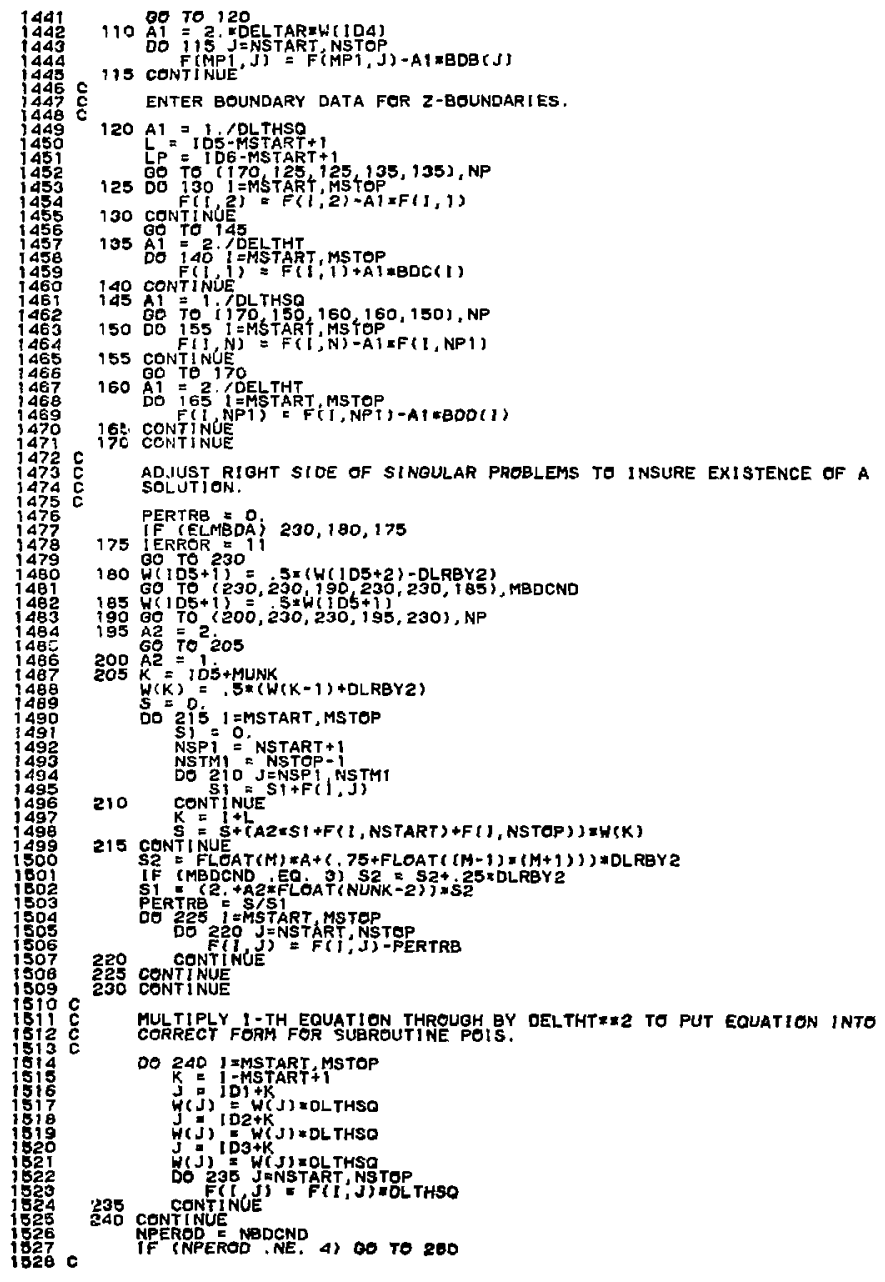




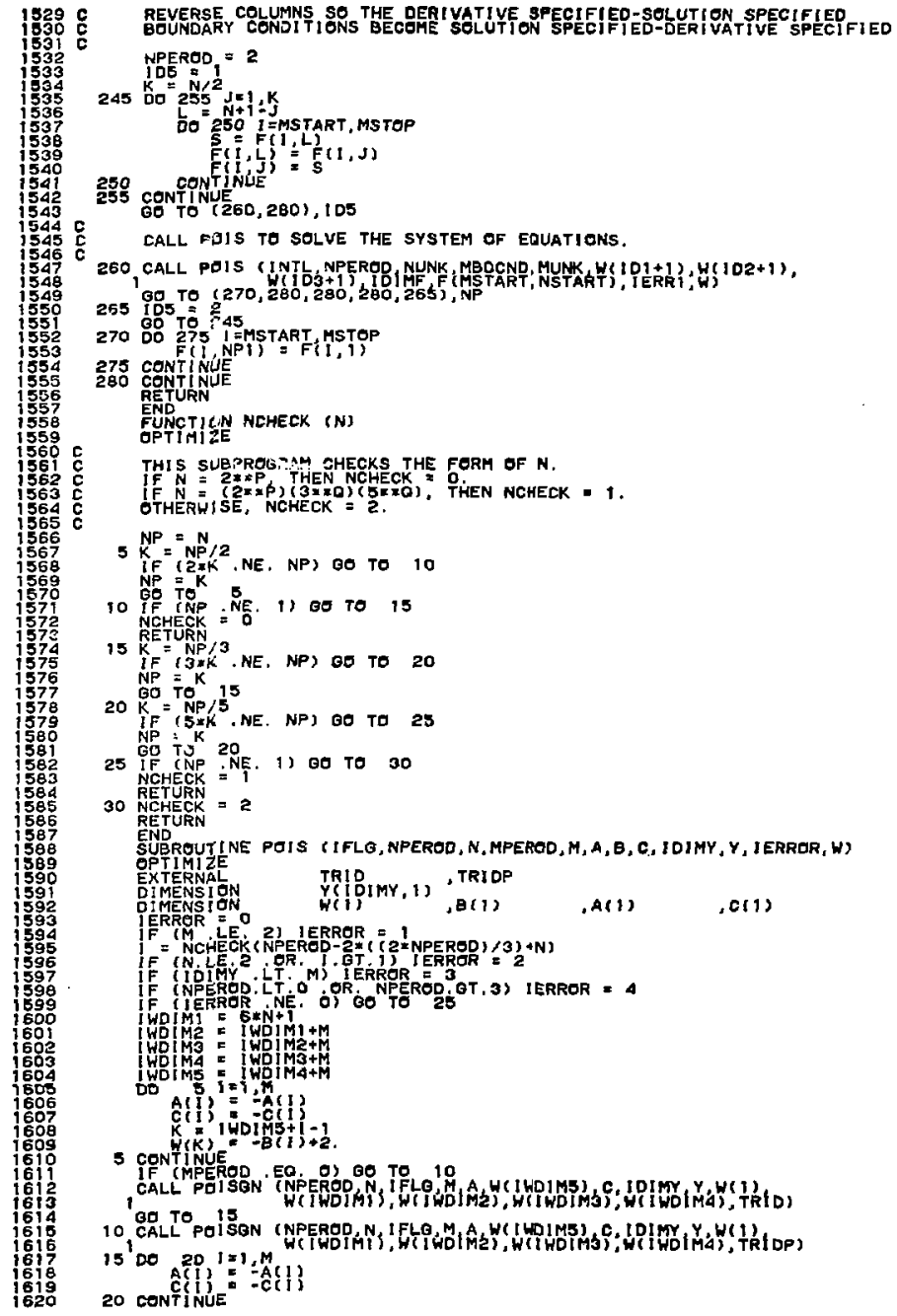




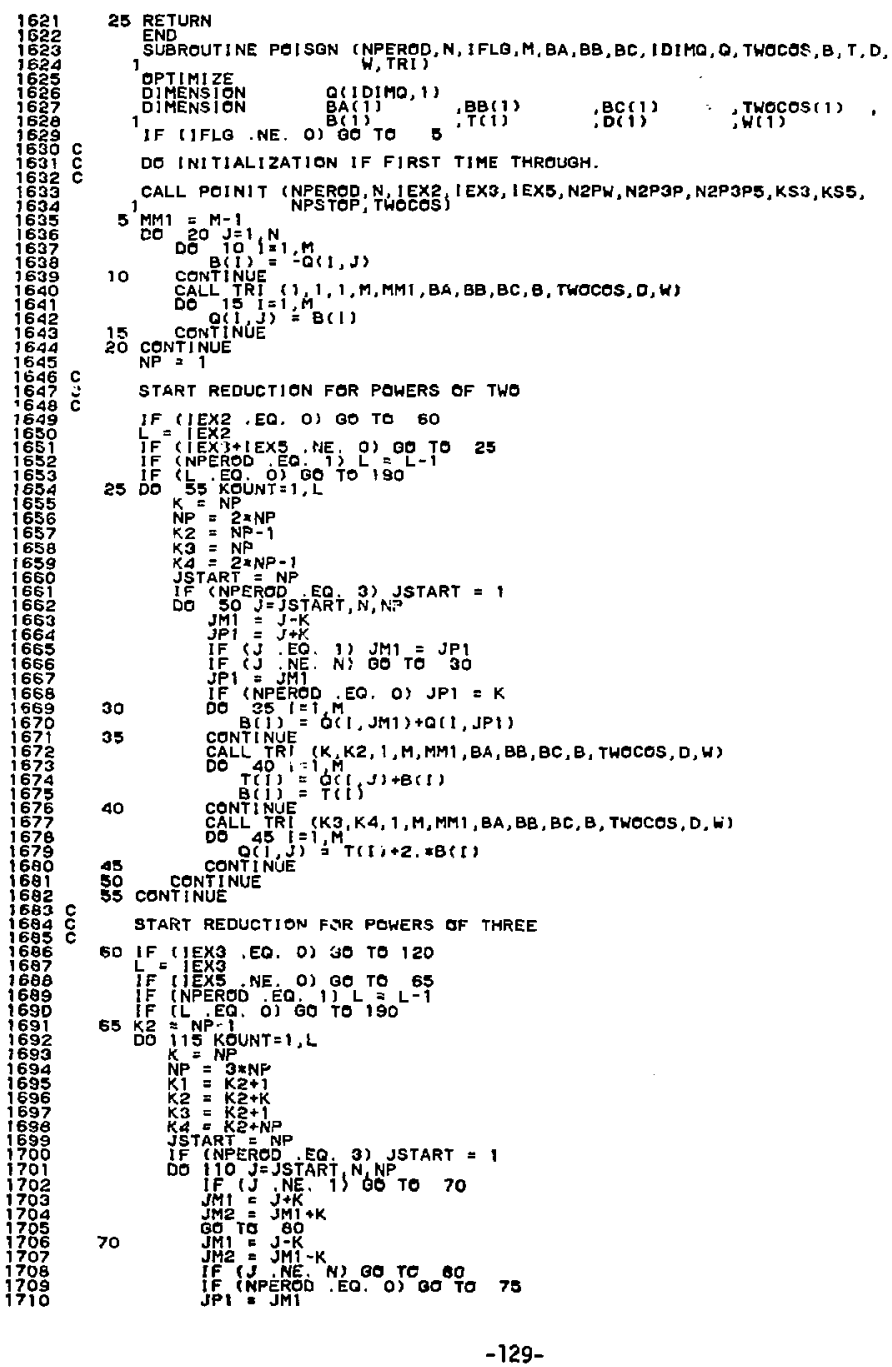




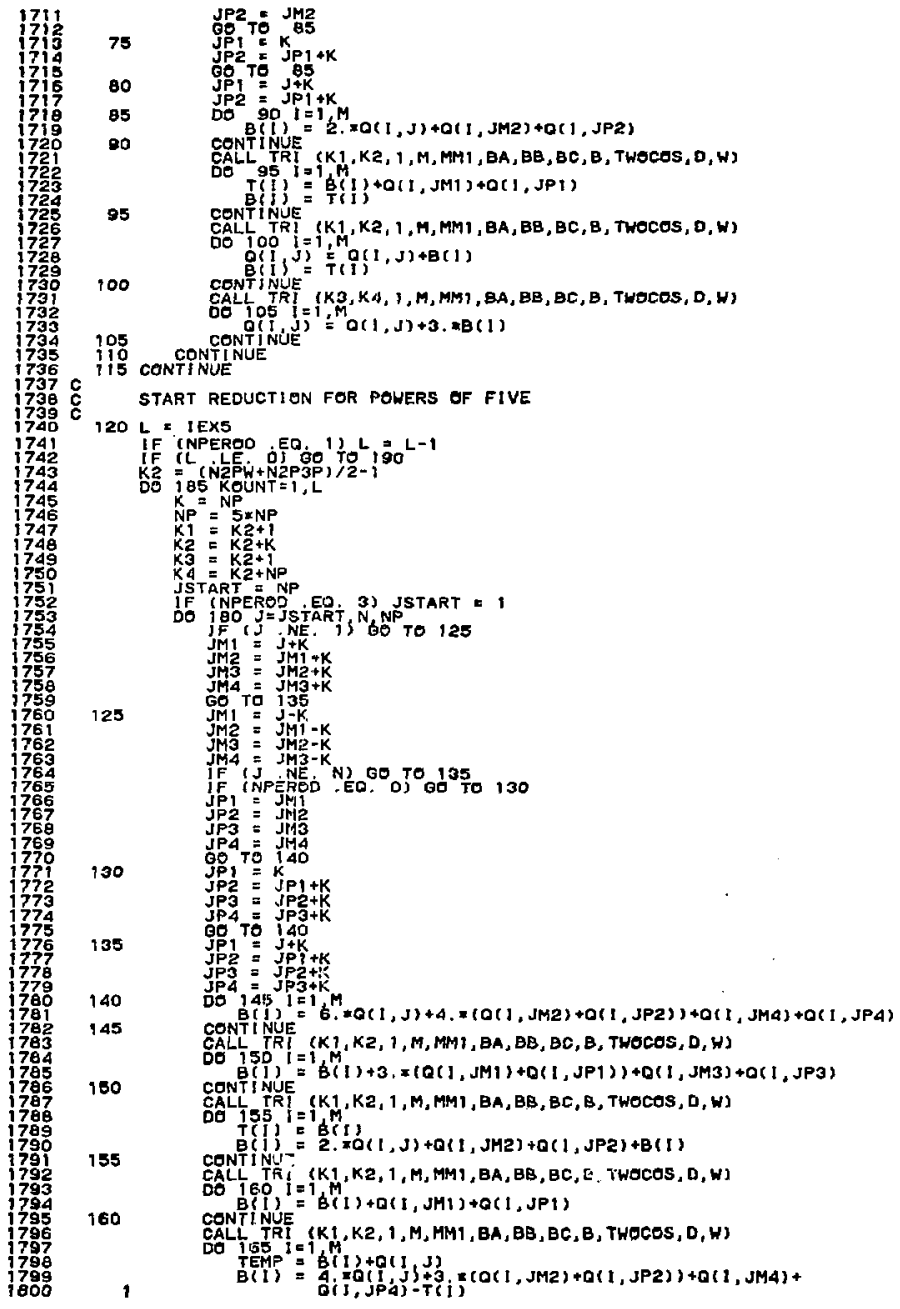




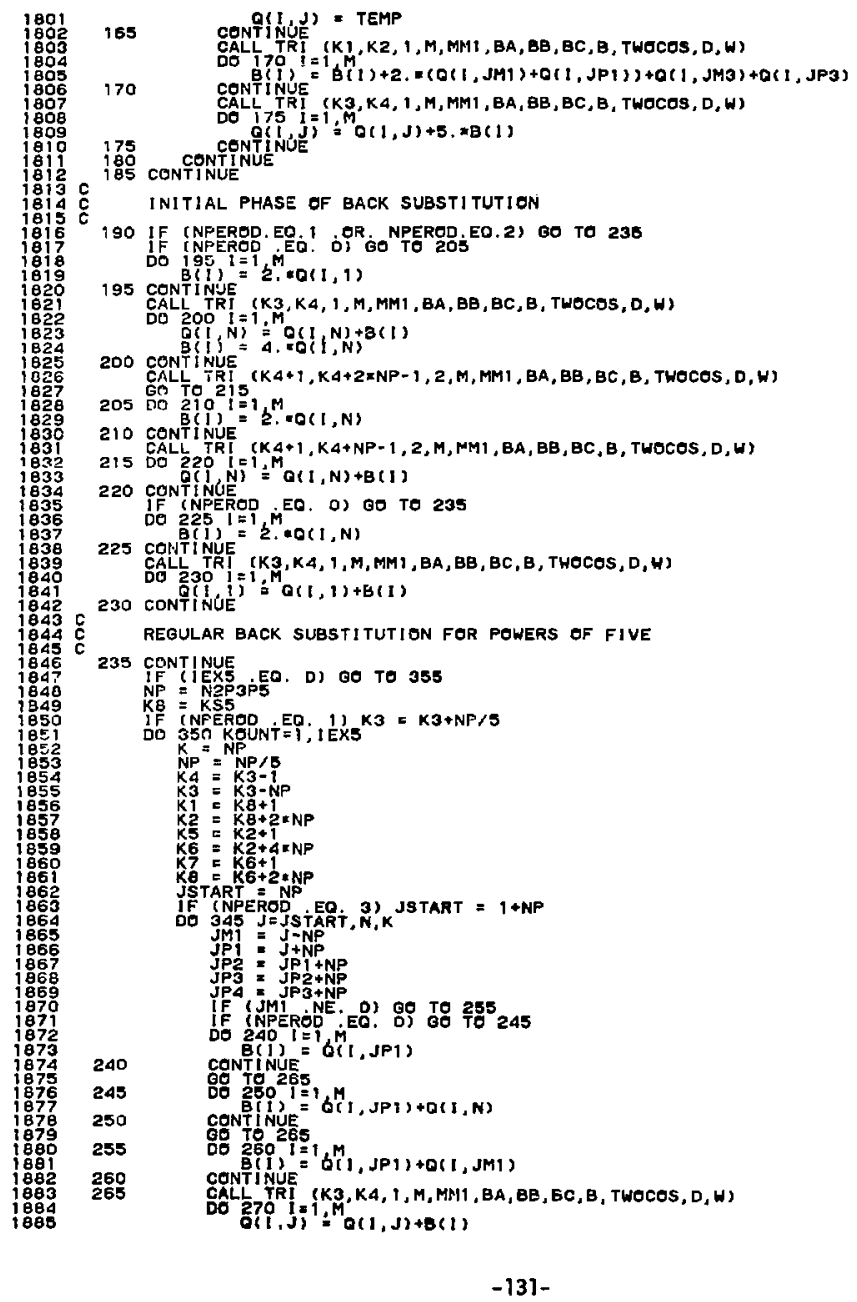




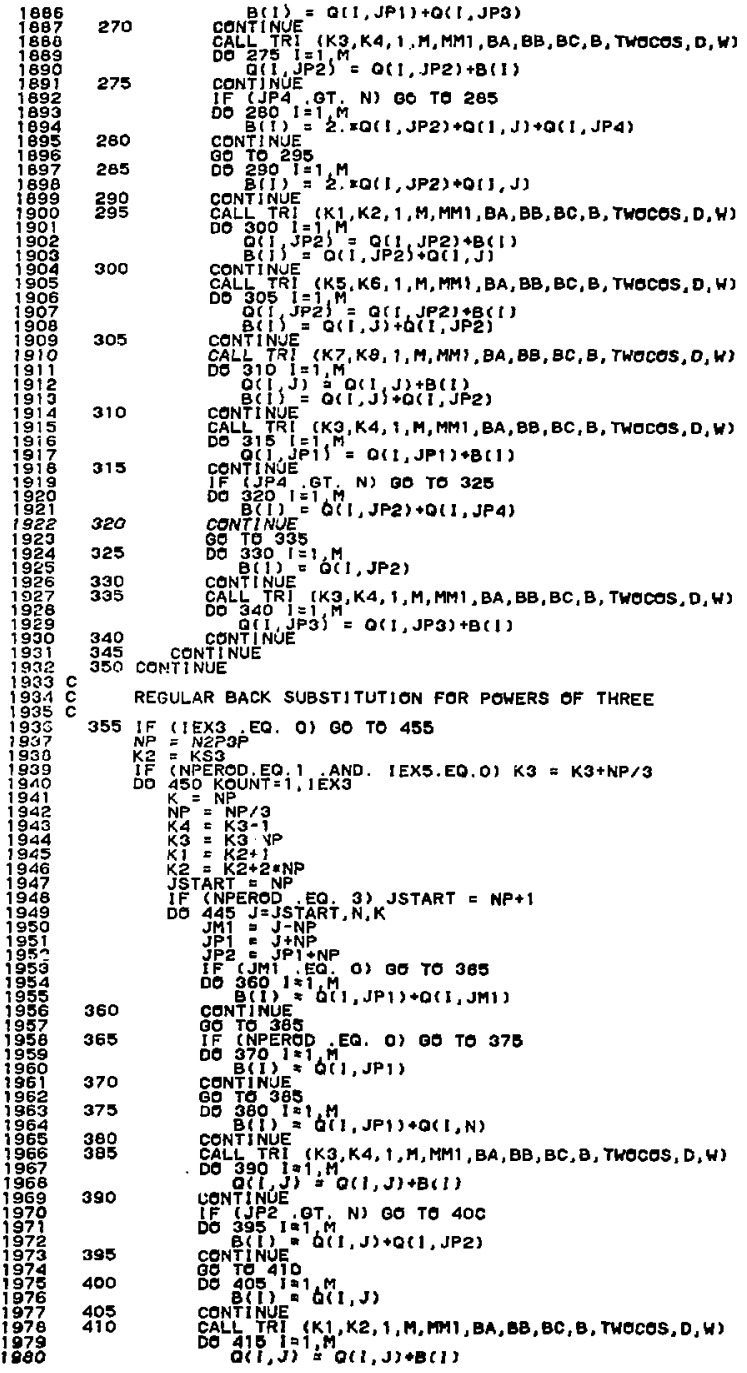




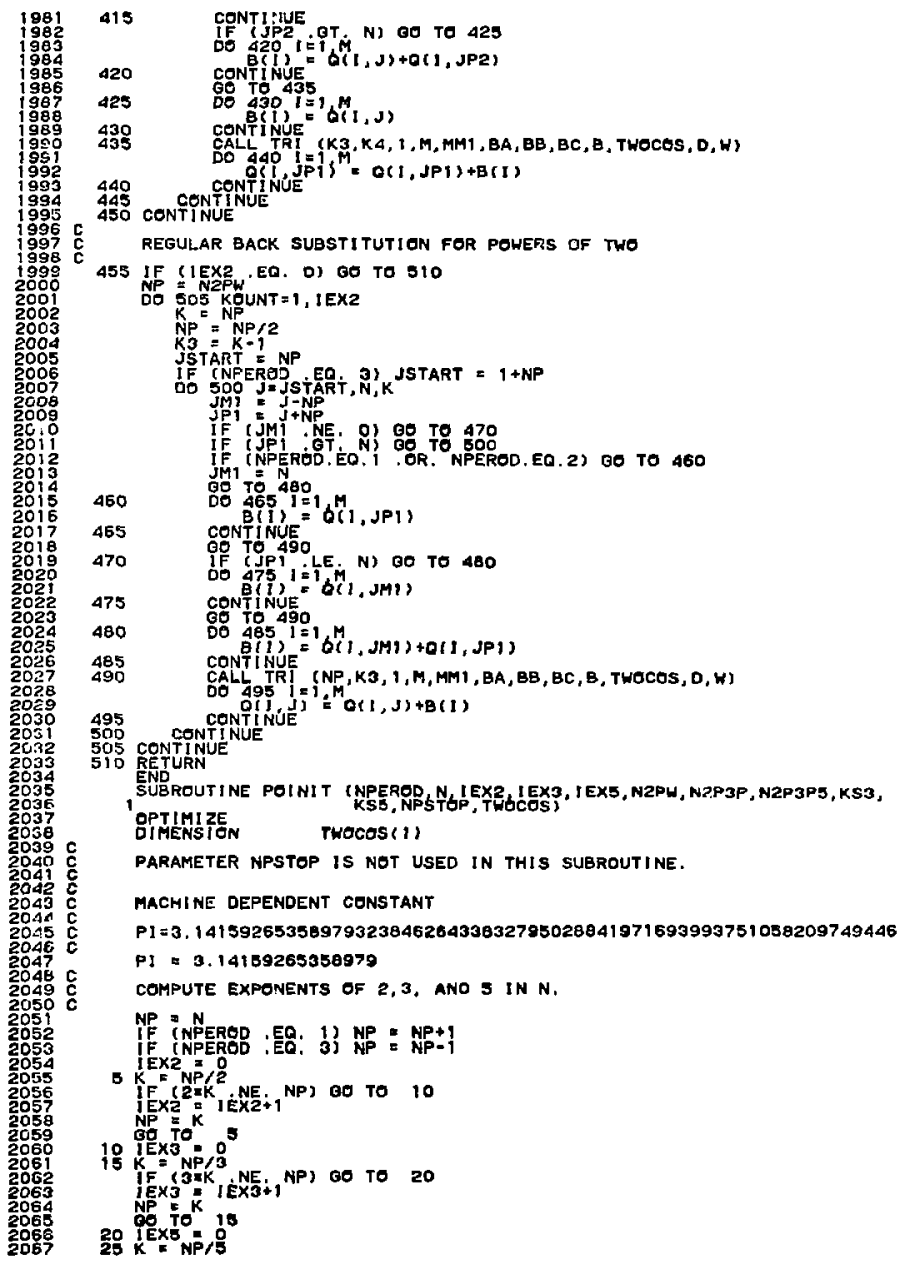




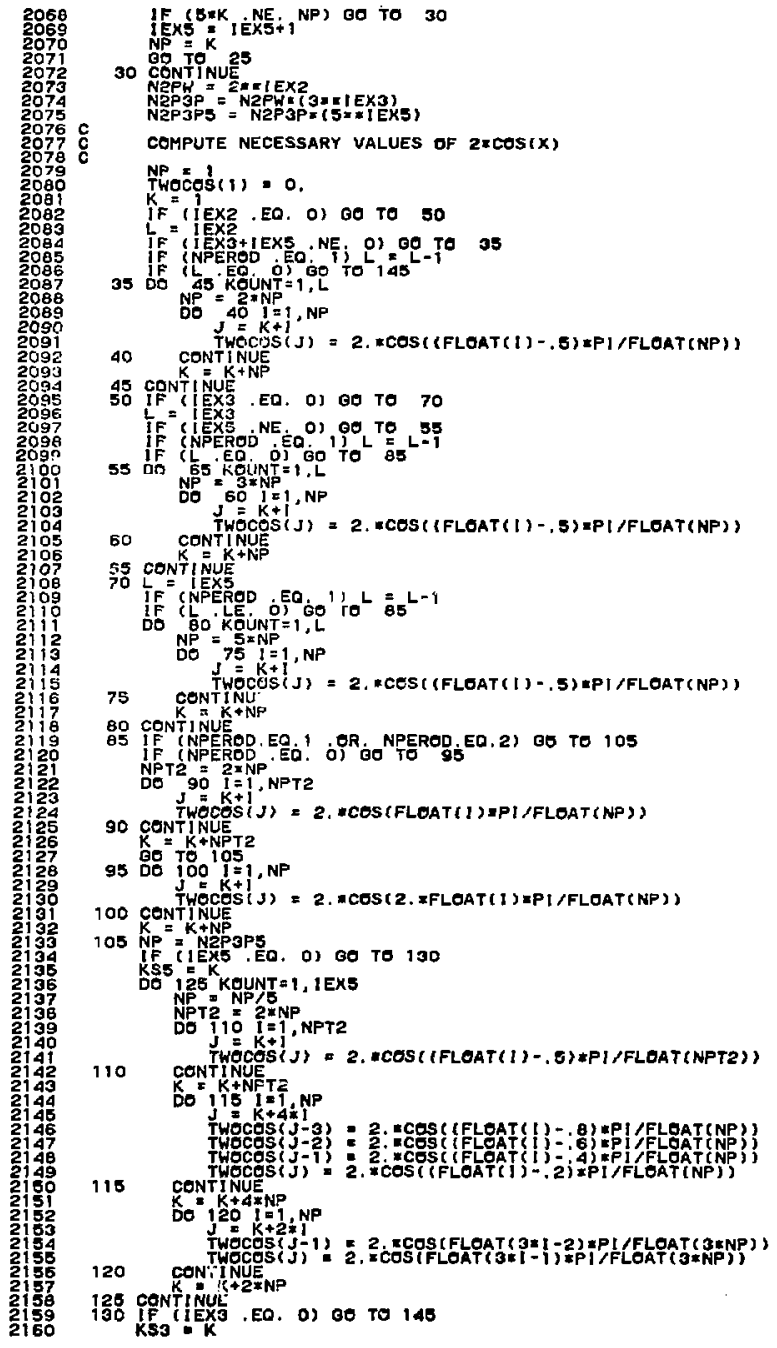




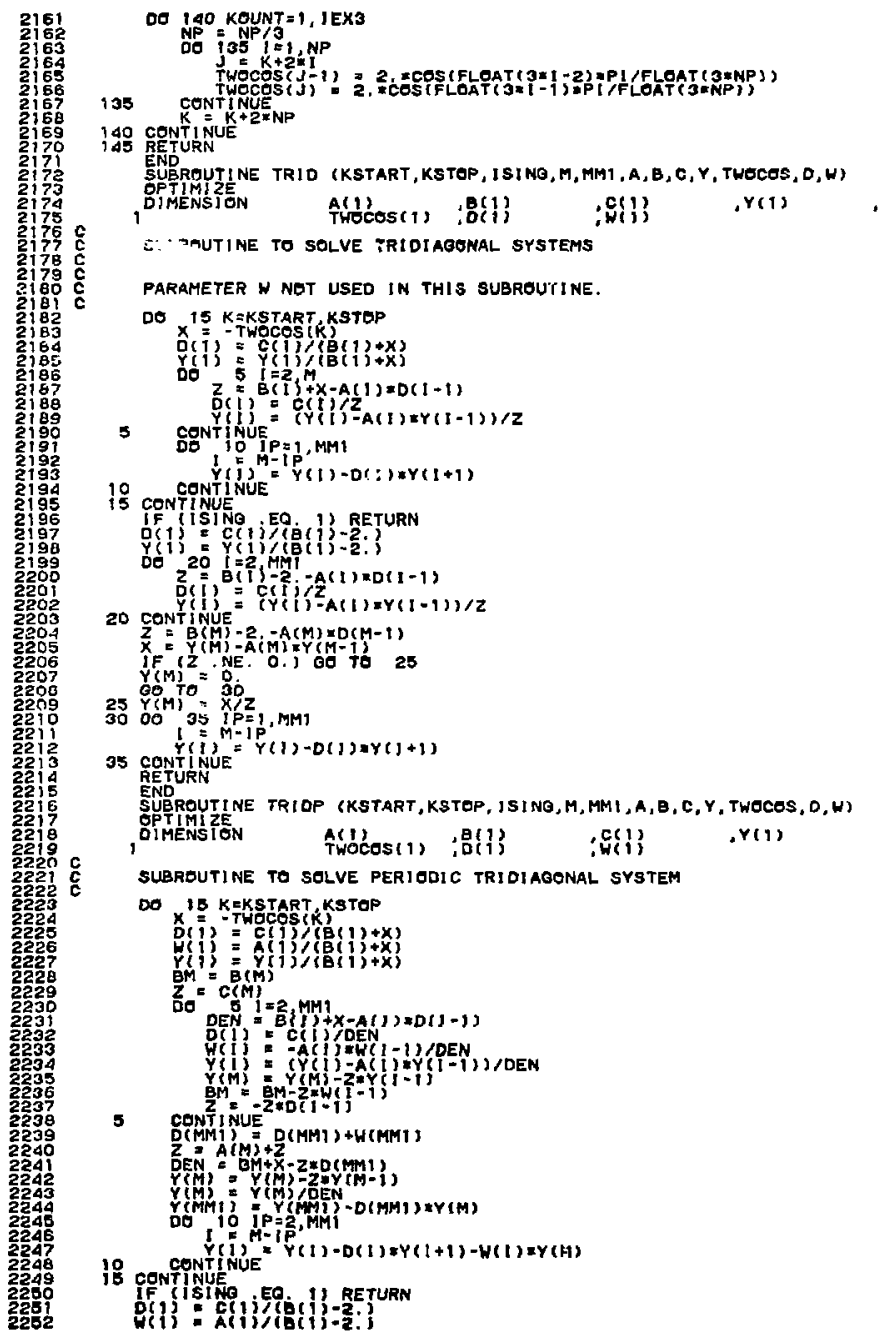




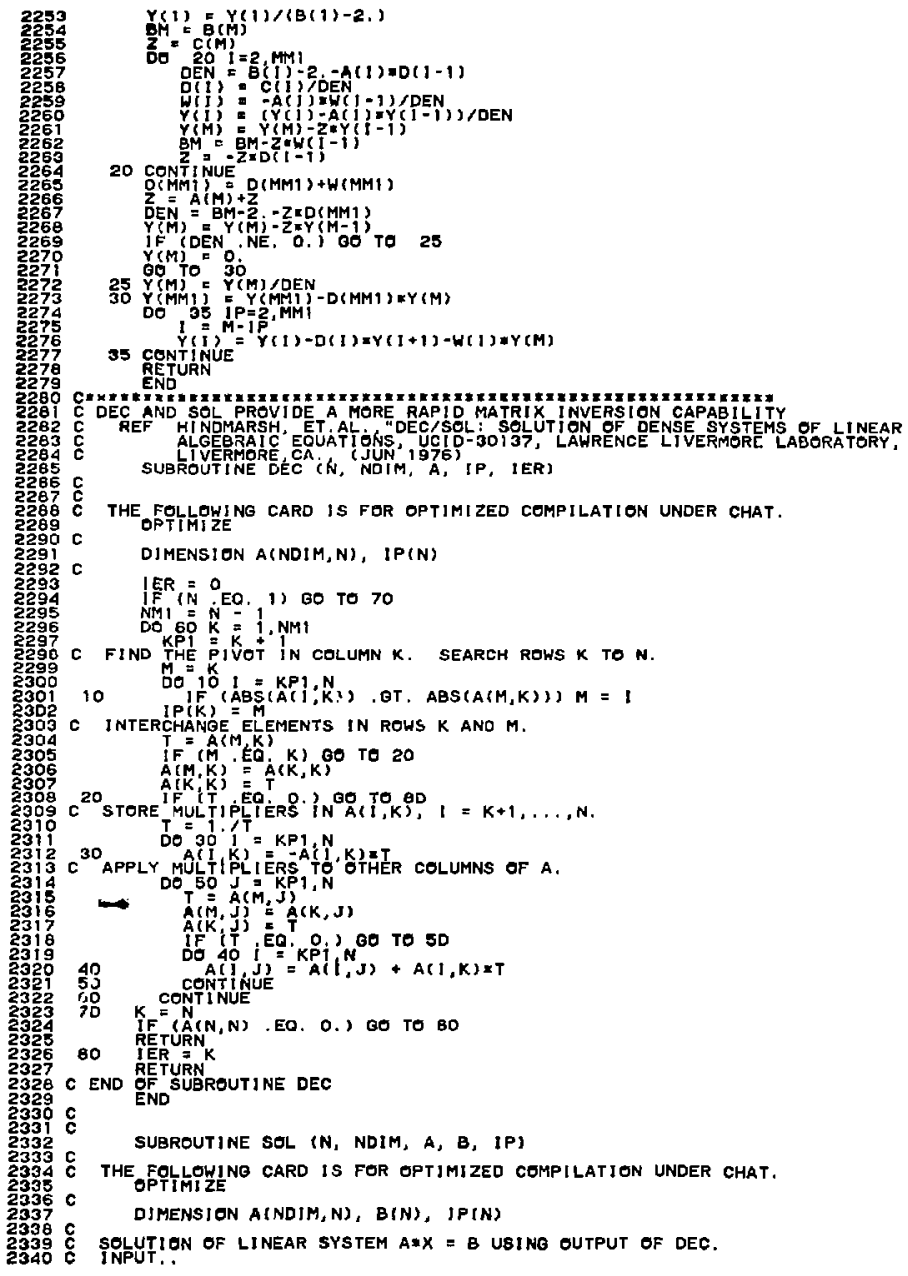




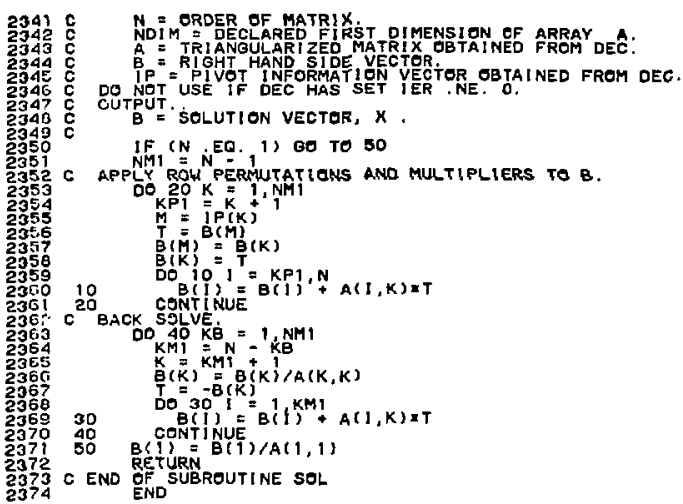


Sample Input

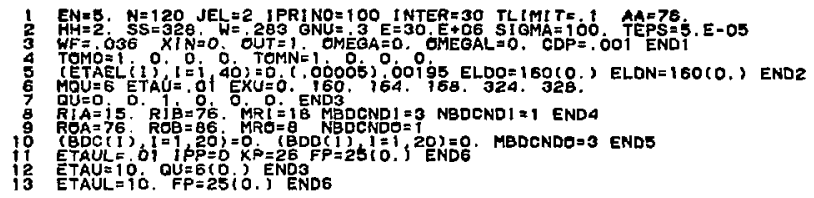




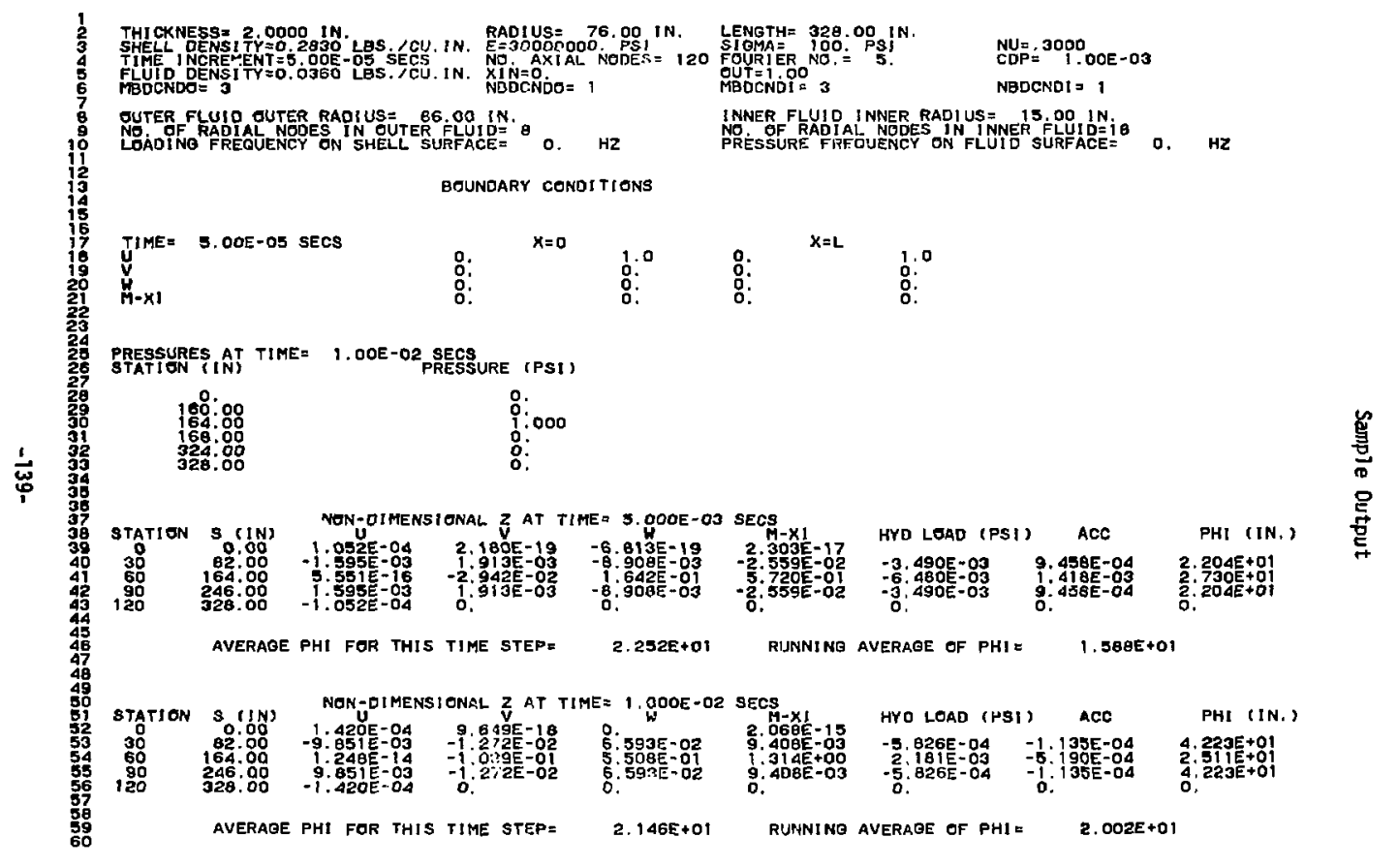




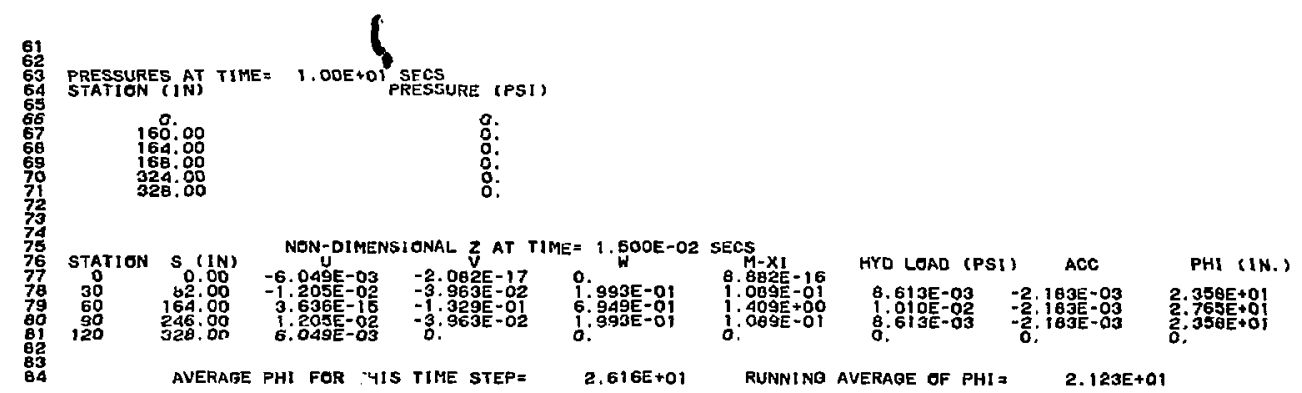

辛 


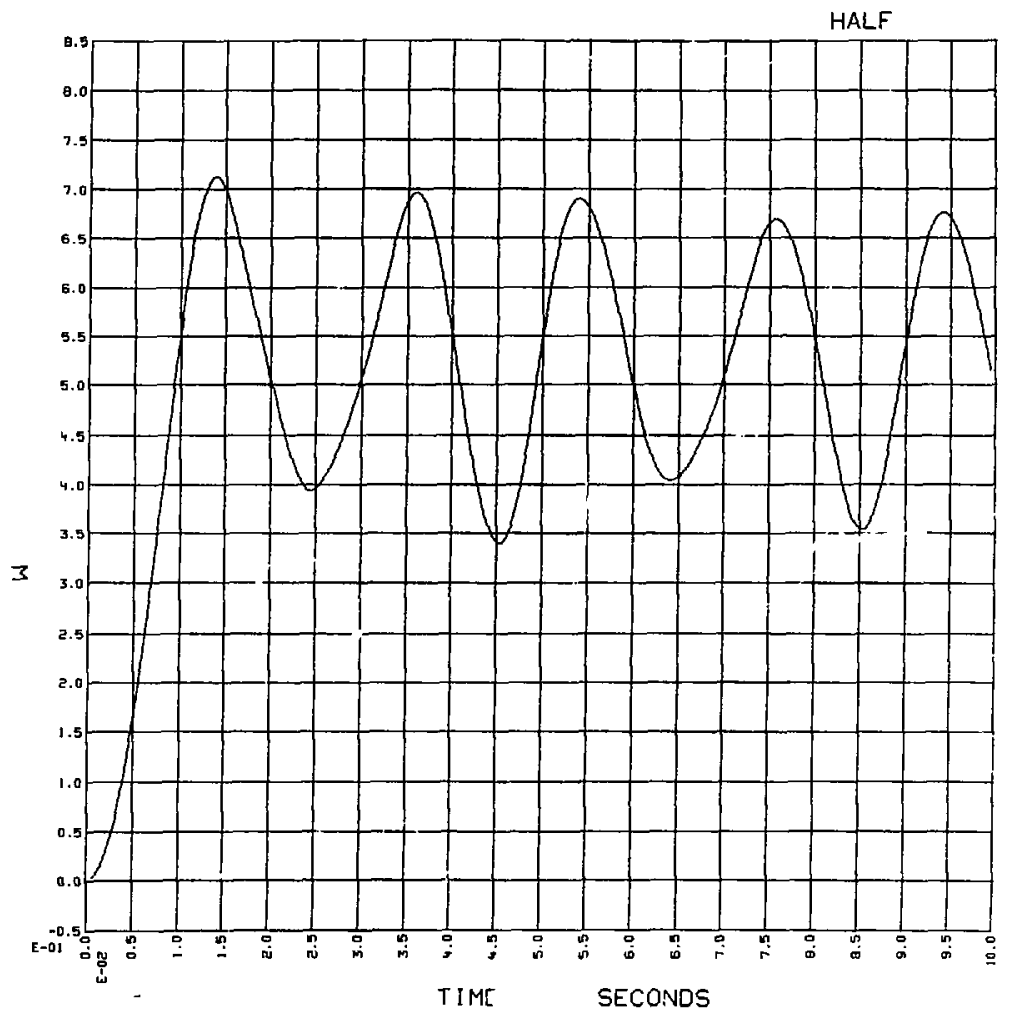

Figure D-1. Time History of Dimension less Radial

Displacement at the Shell's Mid-point 


\section{REFERENCES FOR APPENDIX D}

D-1. Martin, J. T., et. al., "LRLTRAM Language Used with the Chat and Star Compilers," Lawrence Livermore Laboratory Report LTSS-207, Edition 4, (Dec. 1974). 